\title{
Active Flow Control Schemes for Bluff Body Drag Reduction
}

\author{
A Thesis \\ Presented in Partial Fulfillment of the Requirements for the Degree \\ Master of Science in the Graduate School of The Ohio State \\ University \\ By \\ Jacob Whiteman, B.S. \\ Graduate Program in Aeronautical and Astronautical Engineering
}

The Ohio State University

2016

Master's Examination Committee:

Dr. Mei Zhuang, Advisor

Dr. Shawn Midlam-Mohler 
(c) Copyright by

Jacob Whiteman

2016 


\begin{abstract}
Bluff body vehicle drag is dominated by pressure drag on the rear end of the body due to the effect of momentum causing the flow to detach from the body contour. This flow separation results in a pressure difference between the front and back end, making up the pressure drag. A friction force is also generated at the contact of air and solid body that contributes to the total drag, however in the case of bluff body flow this value is far outweighed by the pressure drag. The rear separation region is also dominated by complex time dependent vortices, of which this pressure drag is also dependent, thereby making the overall drag at least partially dependent on the strength and frequency of this shedding phenomena as well. In this study, both a two-dimensional and three-dimensional Ahmed model are used, however only the zero-slant angle case is studied to coincide with the majority of transportation trucks and buses that are on the road today. Numerical simulation experiments on vortex shedding and corresponding drag coefficients from a two-dimensional bluff body are performed over a range of Reynolds numbers from one to four million. The simulations are performed using ANSYS Fluent, specifically the turbulence model of k-epsilon RNG (Re-normalization Group). In order to enhance the accuracy of the shedding wake vortices, an enhanced non-equilibrium wall treatment is utilized. Active control is implemented on the body via velocity boundary conditions in the form of blowing and suction jets. These controls range in velocity from half to double
\end{abstract}


the free-stream inlet velocity. An overall drag coefficient reduction in excess of $75 \%$ is observed for maximum power input to the actuators. In addition, a trend of increasing Strouhal number for each successive increase in actuator power (and corresponding reduction in drag) is noted. Important physical mechanisms involving near-body wake flow are analyzed to determine optimal wake flow pattern and corresponding control schemes. Discoveries are then used to study similar controls on the threedimensional bluff body based on those of the two-dimensional model. For the 3-D simulations the Large Eddy Simulation model is used for the calculation of flow field variables within Fluent, however an introductory RANS analysis is performed as well. Control schemes involving suction jets are investigated. Aspects of the flow pattern such as shedding and streamlines are studied in depth in an effort to determine the most efficient application of the suction controls. These schemes seek to reduce the aerodynamic drag without constraints on the basic design of the model itself. An average of $10 \%$ drag reduction is recorded. 


\section{Acknowledgments}

I would like to acknowledge my adviser, Dr. Mei Zhuang, for her continual guidance through the entirety of my Master's program as well as undergraduate professor here at The Ohio State University. None of this work would have been possible without her knowledge and dedication. Additional insights into the 2D specific flow are also performed by undergraduate intern Tom Ebner. This work was partially funded by the Ohio State University Simulation Innovation and Modeling Center (SIMCenter) through support from Honda R\&D Americas, Inc. In addition, Honda Marysville was intrumental in running some of the more computationally intensive simulations for the 3D models. Special thanks to the Ohio Supercomputer Center and OARnet, which are members of the Department of Higher Educations Ohio Technology Consortium. 


\section{Vita}

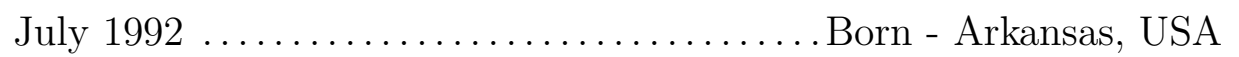

June 2010 ......................... Grove City High School

May 2014 ............................ Aerospace Engineering, The Ohio State University

August 2014 - December 2015 ............ Graduate Research Associate, Department of Mechanical and Aerospace Engineering, The Ohio State University

\section{Fields of Study}

Major Field: Aeronautical and Astronautical Engineering 


\section{Table of Contents}

Page

Abstract .................................... ii

Acknowledgments . . . . . . . . . . . . . . . . . . iv

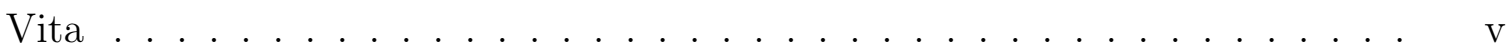

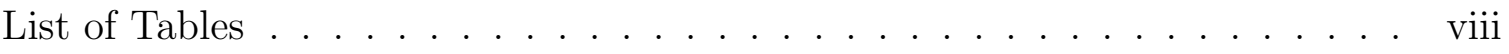

List of Figures . . . . . . . . . . . . . . . . . . ix

1. Introduction . . . . . . . . . . . . . . . . . 1

1.1 Background . . . . . . . . . . . . . . . . . . . 1

1.1.1 Bluff Body Flows . . . . . . . . . . . . . . . . . 2

1.1.2 Control Scheme Advancements . . . . . . . . . . 6

1.2 Motivation . . . . . . . . . . . . . . . 10

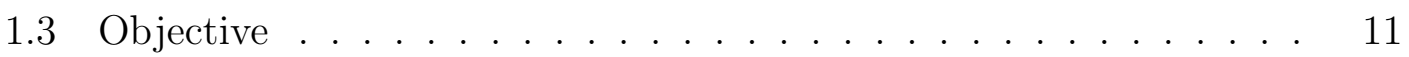

1.4 Thesis Outline . . . . . . . . . . . . . . . . 12

2. Turbulence Modeling and Physical Background . . . . . . . . . . . . 14

2.1 Numerical Turbulence Simulation . . . . . . . . . . . . . . . 14

2.1.1 Large Eddy Simulation . . . . . . . . . . . . . . . . . 14

2.1.2 Reynolds-Averaged Simulation . . . . . . . . . . 15

3. Two-Dimensional Experiments . . . . . . . . . . . . . . . . . . . . . . . . 18

3.1 Simulation Setup . . . . . . . . . . . . . . . . . . 18

3.1.1 Geometry Creation \& Mesh Generation . . . . . . . . . . 18

3.1 .2 Physical Models ... . . . . . . . . . . . 20

3.2 Validation Study . . . . . . . . . . . . . . . . 21 
3.3 Numerical Results and Flow Analysis . . . . . . . . . . . . . . 23

3.3.1 Flow Pattern Structure . . . . . . . . . . . . . . 25

3.3.2 Rear-Model Pressure Coefficient . . . . . . . . . . . . 29

3.3.3 Effects of Control Schemes on Strouhal Number and Drag Coefficient . . . . . . . . . . . . . . . . 31

3.4 Multiple Simultaneous Actuators . . . . . . . . . . . . . . 40

4. Three-Dimensional Simulations _. . . . . . . . . . . . . . . . 47

4.1 Simulation Setup . . . . . . . . . . . . . . . . . 47

4.1.1 Discretization and Boundary Conditions . . . . . . . . . 47

4.1.2 Transient Large Eddy Simulations . . . . . . . . . . . . . 49

4.1.3 Unsteady RANS Simulations . . . . . . . . . . . . 50

4.2 Base Case (no control) . . . . . . . . . . . . . . . . 55

4.2 .1 Time-dependent flow features . . . . . . . . . . . . . . 59

4.3 Suction Control Case . . . . . . . . . . . . . . . . . . . . . 63

5. Conclusions and Outlook . . . . . . . . . . . . . . . . . . . 69

5.1 Concluding Remarks . . . . . . . . . . . . . . . . . . . . . . . 69

5.2 Outlook . . . . . . . . . . . . . . . . . . . 70

$\begin{array}{ll}\text { Appendices } & 72\end{array}$

A. Additional Data from 2-D Cases . . . . . . . . . . . . . . . . . 72 


\section{List of Tables}

$\begin{array}{lll}\text { Table Page } & \text { Page }\end{array}$

3.1 Data from suction simulations on square-back Ahmed body (absence of St entry indicates shedding stoppage) . . . . . . . . . . . . . 32

3.2 Data from blowing simulations on square-back Ahmed body (absence of St entry indicates shedding stoppage) . . . . . . . . . . . . 32

3.3 Data from simultaneous control schemes (absence of St entry indicates shedding stoppage) . . . . . . . . . . . . . . . . 42 


\section{List of Figures}

Figure

Page

3.1 Numerical boundary conditions and general domain dimensions . . . 19

3.2 Near-body structured mesh of computational domain . . . . . . . . . 19

3.3 Verification of numerical model (o Experimental [15], • k- $\epsilon$ RNG ) . .

3.4 Instantaneous vorticity contours of base case in $60 \mathrm{~m} / \mathrm{s}$ flow, (a-b) $k-\epsilon$ RNG (c-d) LES . . . . . . . . . . . . . . . . . . . . . 24

3.5 Transient drag comparison between the two different methods . . . .

3.6 (Upper) Typical periodic drag coefficient (Lower) MATLAB fast Fourier transform of drag coefficient for Strouhal number (Suction actuator at

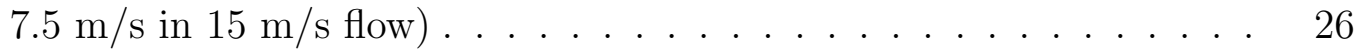

3.7 Shedding of base case at Reynolds number 2.67 million shown with vorticity contours, $\Delta \mathrm{t}=0.02 \mathrm{~s} \quad \ldots \ldots \ldots 27$

3.8 Vorticity contours of steady state (top) suction actuation at $82.5 \mathrm{~m} / \mathrm{s}$ in $55 \mathrm{~m} / \mathrm{s}$ flow (bottom) blowing actuation at $45 \mathrm{~m} / \mathrm{s}$ in $45 \mathrm{~m} / \mathrm{s}$ flow .

3.9 Example plot of pressure coefficient with suction actuator at $\operatorname{Re}=3.66$ million . . . . . . . . . . . . . . . . . .

3.10 Example plot of pressure coefficient with blowing actuator at $\operatorname{Re}=3$ million . . . . . . . . . . . . . . . . . .

3.11 Pressure coefficient of base case at various Reynolds numbers . . . . . 31

3.12 Graphical depiction of drag coefficient data from Table 3.1 . . . . . . 
3.13 Graphical depiction of Strouhal number data from Table 3.1 . . . . . 34

3.14 Graphical depiction of drag coefficient data from Table 3.2 . . . . . . 35

3.15 Graphical depiction of Strouhal number data from Table 3.2 . . . . 36

3.16 Non-shedding pressure coefficient for both actuators . . . . . . . . . . 39

3.17 Drag coefficient data for equivalent power of $0.5 \cdot U_{\infty} \ldots \ldots . . . . . \quad 42$

3.18 Drag coefficient data for equivalent power of $1.0 \cdot U_{\infty} \ldots \ldots . . . . .43$

3.19 Drag coefficient data for equivalent power of $1.5 \cdot U_{\infty} \ldots \ldots 44$

3.20 Drag coefficient data for equivalent power of $2.0 \cdot U_{\infty} \ldots \ldots . . . . \quad 45$

4.1 Original Ahmed model geometry . . . . . . . . . . . . . . . 48

4.2 Numerical boundary conditions for 3D simulations . . . . . . . . . 50

4.3 Multi-view ICEM mesh for numerical simulation . . . . . . . . . . . . 51

4.4 Transient drag coefficient for RANS simulations . . . . . . . . . . 52

4.5 RANS, Time-averaged, plane-projected, streamlines: (1 ${ }^{\text {st }}$ Row) $\mathrm{Re}=$ 0.5E6, ( $2^{\text {nd }}$ Row) Re $=2.78 \mathrm{E} 6[21],\left(3^{r d}\right.$ Row) Re $=4 \mathrm{E} 6 \ldots . . . .53$

4.6 Isosurface of pressure in rear wake colored by velocity magnitude . . . 54

4.7 RANS, Pressure distribution and streamlines projected to the vehicle rear-end surface: (Top) $\mathrm{Re}=0.5 \mathrm{E} 6$, (Middle) $\mathrm{Re}=2.78 \mathrm{E} 6$ (stationary floor) $[21],($ Bottom) $\mathrm{Re}=4 \mathrm{E} 6 \ldots \ldots \ldots$. . . . . . . .

4.8 Surface projected streamlines from different $\mathrm{x} / \mathrm{H}$ locations, left from RANS simulation at $\mathrm{Re}=0.5 \mathrm{E} 6$, right from LES at Re=0.5E6 [21] . . 56

4.9 LES, Time-averaged, plane-projected, streamlines: ( $1^{\text {st }}$ Row) $\mathrm{Re}=$ 0.5E6, ( $2^{\text {nd }}$ Row) Re $=0.5 \mathrm{E} 6[21],\left(3^{\text {rd }}\right.$ Row) Re $=4 \mathrm{E} 6 \ldots . .$. 
4.10 Pressure distribution and streamlines projected to the vehicle rear-end surface: (Top) $\mathrm{Re}=0.5 \mathrm{E} 6,($ Bottom) $\mathrm{Re}=0.5 \mathrm{E} 6[21] \ldots \ldots$.

4.11 Surface projected streamlines from different $\mathrm{x} / \mathrm{H}$ locations, left from LES simulation at $\mathrm{Re}=0.5 \mathrm{E} 6$, right from LES at $\mathrm{Re}=0.5 \mathrm{E} 6[21] \ldots$

4.12 Unsteady total pressure signal from monitor points 1 and $2 \ldots \ldots 61$

4.13 Fast Fourier Transform of monitor points 1 and $2 \ldots \ldots 62$

4.14 Instantaneous reverse flow plot isosurfaces in the vehicle wake colored by pressure (airflow inside shaded region has negative $\mathrm{x}$-velocity) . . .

4.15 Q-Criterion iso-surfaces in the wake of the vehicle model, colored by local velocity magnitude . . . . . . . . . . . . . .

4.16 Suction Control Scheme Implementation . . . . . . . . . . . . . . 64

4.17 LES, Time-averaged, plane-projected, streamlines: (Top) Re $=4 \mathrm{E} 6$ (no control), (Bottom) $\mathrm{Re}=4 \mathrm{E} 6$ (suction) $\ldots \ldots \ldots \ldots$. . . .

4.18 Instantaneous reverse flow plot isosurfaces in the vehicle wake colored by pressure (airflow inside shaded region has negative $\mathrm{x}$-velocity) . . .

4.19 Pressure distribution and streamlines projected to the vehicle rear-end surface: (Top) Re $=4 \mathrm{E} 6$ (no control), (Bottom) Re $=4 \mathrm{E} 6$ (suction control $\ldots \ldots \ldots \ldots \ldots \ldots$. . . . . . . . . . . . 6 67

4.20 Transient drag coefficient for LES simulations . . . . . . . . . 68

A.1 Strouhal number data for equivalent power of $0.5 \cdot U_{\infty} \ldots \ldots . \ldots 73$

A.2 Strouhal number data for equivalent power of $1.0 \cdot U_{\infty} \ldots \ldots . .73$

A.3 Strouhal number data for equivalent power of $1.5 \cdot U_{\infty} \ldots \ldots \ldots$

A.4 Strouhal number data for equivalent power of $2.0 \cdot U_{\infty} \ldots \ldots \ldots$

A.5 Strouhal number data for equivalent power of $0.5 \cdot U_{\infty}$ at $\operatorname{Re}=2.33 \mathrm{E} 06 \quad 75$

A.6 Strouhal number data for equivalent power of $1.0 \cdot U_{\infty}$ at $\operatorname{Re}=2.33 \mathrm{E} 06 \quad 75$ 
A.7 Strouhal number data for equivalent power of $1.5 \cdot U_{\infty}$ at $\operatorname{Re}=2.33 \mathrm{E} 06 \quad 76$

A.8 Strouhal number data for equivalent power of $2.0 \cdot U_{\infty}$ at $\operatorname{Re}=2.33 \mathrm{E} 06 \quad 76$ 


\section{Chapter 1: Introduction}

\subsection{Background}

Bluff body flow is a very relevant and expansive area of engineering research in today's emerging research scene. Everything from flow around tall city buildings to small smart cars is being addressed. Throughout the last few decades the main focus on ground vehicle improvisation has been through aspects such as engine efficiency, economy, and impact on the environment as a means of reducing fuel or production costs. However, due to the recent increase in numerical capabilities it is now possible to model such a vehicle virtually in order to gain insight into a new form of vehicle efficiency; aerodynamic drag reduction. In this way, a reduction in aerodynamic drag will cause an effective increase in fuel economy and corresponding reduction in emissions. In fluid mechanics these cars and trucks can be approximated as simple bluff bodies in ground proximity, with additional complex features such as sharp edges or cavities as the need may be. However, due to the time dependence of the solution, turbulence, and Reynolds numbers in the millions, these simulations require specialized care and are extremely challenging. 


\subsubsection{Bluff Body Flows}

Subsonic aerodynamic research has its main focus centered on determining the forces acting on the body at all points on the surface. For streamlined bodies such as an airfoil or wing the research is focused mainly on lift generation. Since the flow stays attached for much of the surface area the drag due to friction is much more dominant than that from pressure, giving pressure drag only secondary importance. For the case of bluff body flow, in contrast to that of a streamlined body the overall aerodynamic drag is in fact dominated by this pressure drag and friction drag is of secondary concern.

When flow passes over bluff bodies an excess of momentum in that flow will cause it to detach from the body at the abrupt end, formulating large separated regions over a wide range of Reynolds numbers. This wake is then characterized by periodic and asymmetrical shedding of formed vortices. Specifically, this shedding frequency is quantified by a non-dimensional number known as the Strouhal number:

$$
S t=\frac{f_{s} L}{U}
$$

where $f_{s}$ is the shedding frequency, $L$ is the characteristic length, and $U$ is the characteristic velocity of the fluid. As a result, for small Strouhal numbers $(\sim 0.01)$ a quasi-steady state is achieved in the flow and for large numbers $(\sim 10)$ the flow is dominated by viscosity. However, at intermediate values of Strouhal number the oscillations are characterized by inertial separations with a non-quasi steady behavior $[1]$.

A classical example of these separations and the corresponding Strouhal numbers is presented by Roshko [2] in a paper detailing flow past a circular cylinder at very 
high Reynolds numbers. In this study, experiments were done on a large circular cylinder in a wind tunnel at Reynolds numbers of $10^{6}$ to $10^{7}$. It was revealed that at a Reynolds number of 3.5E6 the drag coefficient became constant and distinct vortex shedding occurred with a specific Strouhal number, determined via hot-wire anemometry to be 0.27 . This value of $3.5 \mathrm{E} 6$ was termed the critical Reynolds number, as below this value vortex shedding was not observed. These findings defined what is called a Reynolds number effect which involves aerodynamic characteristics such as Strouhal number, drag coefficient, or lift coefficient, being dependent on higher or lower Reynolds numbers. However, it was also discovered that an increase in shedding frequency was accompanied by a decrease in drag, and vice versa. Finally, a splitter plate was inserted into the flow at the rear of the cylinder which had the added effects of suppressing the shedding and causing a small decrease in drag at higher Reynolds numbers. Further work has also been done in a similar capacity [3-5].

Presented next is work done by Almeida [6] on the flow past rectangular cylinders. Throughout this work the Large Eddy Simulation method is employed to study unsteady incompressible flows around rectangular cylinders with side ratios varying from one to ten. With the cases running at Reynolds numbers below 10000, power spectral density signals from velocity were used to determine Strouhal numbers from Fourier transforms between zero and two. The numerical visualization of the vorticity field is used to understand the Strouhal number behavior. Firstly, vortices formed on the face of the cylinders advected downstream and interacted with those formed in the rear. This interaction was stronger as the aspect ratio increased. Additionally, the amount of vortices observed on the cylinder face increased with the aspect ratio. 
Combining these two observations, the author made the conclusion that the increasing number of vortices and strength of interaction for each increase in aspect ratio caused the corresponding increase in overall rear Strouhal number.

Extensive research has been performed on these kinds of bluff body flows [2-6], but also on how to locally control the flow in an attempt to remove or delay the development of the recirculation zone and the separated vortices [7-10]. Specifically, work done by Kozlov [7] involved the effect of single dielectric barrier discharge plasma actuators as an efficient means of flow control on cylinders at Reynolds numbers of up to 164000 . Smoke generators and PIV measurements were used to show the largescale separated flow region accompanied by unsteady Karman vortex shedding at a Strouhal number of 0.2 . However, the plasma actuators were shown to substantially reduce the extent of the separated flow region and the associated Karman vortex shedding was completely eliminated. With the plasma on, a drag reduction of $90 \%$ was noted at the lowest Reynolds numbers tested (5000), however this effect quickly became less prominent as Reynolds number increased. This leads to the conclusion that control of the shedding has a substantial impact on drag coefficient. Similar conclusions were reached by Do [8] as well.

\section{Generic Automobile Model}

Before the dawn of numerical modeling, some experimental work was done on simplified vehicle models. For example, S.R. Ahmed researched the wake structure of such a model in close non-moving ground proximity [11]. It was at this point that the term "slant-angle" was created to represent the angle of the rear end of the model, from horizontal. It was discovered that the wake could be characterized by a separation bubble and these vortices forming at every slant-angle tested, in addition to 
a horseshoe vortex system longitudinally. Recorded drag coefficient values also seemed to indicated a trend for increasing slant angle. Following this trend Ahmed [12, 13] performed another study detailing the specific differences noted in rear circulation for several specific slant angles. Although a pair of horseshoe vortices placed one above the other in the "separation bubble" were seen for all different slant angles, the strength and location of these vortices was dependent on slant angle.

Investigations into this shedding further by Morel [14] were performed on a similar vehicle-like model in ground proximity, however keeping the slanted surface and body volume constant for comparability. This study revealed that there was a certain slant angle at which the drag coefficient saw a large spike in magnitude, noted as the "critical angle". This coincided with the pattern of vorticity generation in the wake of the model being different for each set of slant angles. Two specific slant angles on either side of this proposed critical angle were measured by Lienhart [15]. Detailed LDA and flow visualization measurements were performed and their respective flow topologies documented, in an effort to not only explain this change in drag but also to act as a reference for validation of computational simulations. Similar drag coefficient results were seen by Strachan [16] experimentally using a similar model. In a numerically focused paper by Gillieron [17], the changes in vortex wake airflows and aerodynamic drag coefficients during transitions from two-dimensional base behavior to three-dimensional hatchback behavior and back again are detailed. Computed results suggested that the flow topology associated with the separation bulb on the rear window varies continuously with the slant angle. 


\subsubsection{Control Scheme Advancements}

The separation of boundary layer flows has been an Aeronautics engineer's main focus since the inception of fluid mechanics. The need to alter the naturally occurring flow field to a more beneficial state has been accomplished by both inhibition and excitation of separating flows [7-10, 18-22]. Separation control in the specific case of bluff bodies can be described by three main philosophies; delaying the onset of separation and vortices formation, reducing the general magnitude of the shedding, or breaking apart the vortical structures. For example, in a two-pronged study Brunn [19] controlled the flow around an Ahmed body with a slant angle of $35^{\circ}$ by use of periodic excitation using a synthetic jet along the upper window section. The study utilized both experimental and simulational work and indicated that a drastic reduction of recirculation area can be achieved with proper controls at a wide range of Strouhal numbers.

Common terminology typically used when discussing these types of controls involves the distinction between active and passive control schemes. Although a formal definition does not exist, usually if a device does not require additional energy input it can be classified as a passive control. Conversely, active controls require additional energy to be supplied from outside the system in order to function. In the following sections several of these types of devices are explored in both the experimental domain and the computational domain. 


\section{Passive Controls Testing}

Passive controls can include modifications of the body itself, or more probably exterior modifications on the body, and range from simple geometric alterations to extensive attachments. Bruneau [23] implemented passive control on a two-dimensional square-back Ahmed model in the form of porous layers attached in various positions around the body. Numerical simulation showed that for certain symmetrical configurations of porous slices, the drag coefficient could be reduced by $45 \%$. The reduction in drag was attributed to the rise of static pressure and the decrease of vorticity measured at the back.

An extensive parametric study by Beigmoradi [18] involved altering many different geometric features of the original Ahmed model, including slant angle but also rear box length, angle and boat tail angle. Numerical simulations in addition to artificial neural networks and genetic algorithms allowed the study to extend the small number of cases actually run to interpolate hundreds of possibilities. A RANS (ReynoldsAveraged Navier-Stokes) turbulence model was used for numerical simulation and the optimal case (aerodynamically) depended on a combination of all parameters. It was observed that by increasing boat tail angle and rear box length the drag coefficient decreased, however the magnitude depended strongly on the specific slant angle in combination. Similar studies by Khalighi [24] and Hsu [25] involved experimental and numerical testing of similar boat tail configurations, also resulting in decreased drag coefficients.

In a paper by Thacker [10], a new passive control device was presented for wakedrag reduction in flow over a two-dimensional bluff body with a blunt trailing edge. Small tabs were attached to the upper and lower trailing edges of the surface to 
perturb the wake. Both wind tunnel experiments and large eddy simulations are performed at Reynolds numbers up to 80000. An increase of back pressure of up to $30 \%$ was noted resulting in equivalent drag reductions. The LES (Large Eddy Simulation) results attributed the success to the tabs ability to introduce a span-wise mismatch in the vortex-shedding process, resulting in a substantial reduction of the vortical strength in the wake and significant increases in vortex formation length and width.

\section{Active Control Schemes}

Active control schemes usually consist of various suction or blowing jets, in addition to the plasma actuators detailed above. In the cases of the angled Ahmed body controls, the main focus has been to keep air attached to the slant as it flows over. For example, in a study by Roumeas [26] the Lattice Boltzmann numerical method is used to simulate flow over a three dimensional Ahmed model with continuous suction at the top of the rear window, perpendicular to the slant. A parametric analysis showed that reattachment was facilitated by the suction controls at only 0.6 times the free-stream. This reattachment prompted a reduction in wake cross section and an increase in base static pressure, leading to associated drag reductions of $17 \%$. Another approach by Brunn [19] involved both experimental and simulational work on the rear slant in both $35^{\circ}$ and $25^{\circ}$, however instead of suction jets, blowing actuators were used. For the $35^{\circ}$ case, these controls were placed in the same position as Gillieron and were aimed at amplifying the spanwise vortex structures in the separated shear layer to increase the growth of the vortices and intensify the entrainment process. On the other hand, for the $25^{\circ}$ case, steady blowing jets were integrated into the slant corners of the Ahmed body. For the higher angled window the controls 
achieved a reduction of the reverse flow region, however at the same time development of stream-wise vortices generated at the slant side edges was observed. Conversely, for the lower angle the side vortices were weakened by the blowing approach but an increase in separated flow over the slant was noted. A combined flow control approach was suggested for the future.

In the case of a square-back Ahmed body multiple studies have been done involving jets at the rear of the model. In particular Roumeas [26] performed 3D numerical simulations using the Lattice Boltzmann method and flow control utilizing continuous blowing jets distributed around the base periphery. A significant drag reduction of $29 \%$ is recorded with a blowing velocity 1.5 times that of free-stream, however when efficiency is studied the maximum efficiency is achieved at 0.5 times free-stream and an angle of $45^{\circ}$ corresponding at a drag reduction of $20 \%$. In a similar study by Eichinger [21] the same square-back Ahmed model was tested using LES simulations with similar active blowing controls. After an efficiency study an angle of $45^{\circ}$ was also considered the most efficient scheme at $18 \%$ drag reduction. Similar results were obtained in a study by Peres [27] as well.

Bruneau [20] also presented another study involving the coupling of the porous layers described earlier with active controlled blowing jets on the rear of the squareback 2D Ahmed model. Parametric analysis of different jet placement and power was analyzed against rear pressure coefficient plots and drag coefficient. The configuration chosen involved a porous layer on the roof which was seen to split the upper shedding vortex into smaller eddies, however the large bottom shedding vortex remained. The idea was to use a closed-loop blowing jet to push it down in the wake. The coupling of 
both controls allowed a $31 \%$ reduction in average drag coefficient and corresponding increases in pressure coefficient at the rear.

\subsection{Motivation}

The design of ground vehicles is a very complex task, and it is usually the case that what is desirable from an aerodynamic standpoint conflicts with the interests of other designers and manufacturers. In the end, aerodynamic design of a vehicle is a compromise and the aerodynamic engineer must be able to provide either an improved vehicle shape or an alternative way to reduce the aerodynamic drag. The latter solution is the case with which this study is based around. In order to evaluate the actuators used here, the typical model chosen is that of the Ahmed model with a square-back slant angle on the rear end. This is done because this kind of vehicle is most relevant to the commercial shipping industry, which employs many of these tractor-trailers throughout the world. In addition, the choice to utilize active control actuators will eliminate the need for external model changes, as well as be easily adjustable for different flow conditions the vehicle may experience. Investigations into how the flow structure can be changed, and how these flow structure changes can bring about drag reduction also play a major role in this thesis.

Many different studies have already been conducted, and only a fraction are presented here. However, much of the work done in this particular field is focused on a single new idea or one instance of an interesting flow phenomenon. Much of the interactions between various variables such as vehicle shape, Reynolds number, or control schemes are still unknown. One specific motivation for this work is to uncover some of these relationships via parametric experiments involving multiple different 
variables. These types of simulations should reveal how each new addition effects the flow field, including the interactions with previously defined characteristics.

\section{$1.3 \quad$ Objective}

For this thesis, the objectives are outlined as follows. An extensive parametric study is performed on the Ahmed model, involving changing control schemes and Reynolds numbers. The effect of the suction and blowing jets on the flow field around the Ahmed model is analyzed. This also allows for investigation into the Reynolds number effects on the drag coefficient, Strouhal number, and control schemes. The ultimate goal of this study is to reduce the overall drag coefficient on the model by means of developing effective actuators for flow control, however additional contributions are made by detailing the interactions of these various parameters with the model and with each other.

The ability to use available computational resources to solve practical engineering problems is addressed by developing a robust and accurate method of fluid flow simulation. Both RANS and LES are utilized, however it is important to verify the results from RANS against that from LES in order to show the simulation method is realistic. Success will be determined not only by the magnitude of which the drag is reduced, but also by the fluid interactions seen by the specific numerical methods used and the analysis of accompanying fluid dynamics.

\section{Zero-Slant Angle Model}

For the entirety of this thesis the only slant-angle focus will be at the angle of $0^{\circ}$ or $90^{\circ}$, also known as the square-back case (which it will be herein referred to). Previously, research on how the slanted form of the car model effects the drag is 
presented, however many issues regarding these flows are similar to the square-back case. In reference to Chapter 1 the top priority is to reduce overall drag on the vehicle, and the square-back geometry is the most relevant due to it being the most prevalent in vehicles such as large commercial trucks and buses.

The zero-slant angle model also has a secondary significance that involves comparisons between the two-dimensional and three-dimensional flow fields. As mentioned in Section 1.1.1, the flow around an Ahmed body takes different forms according to the angle of the rear slant. However for a zero-slant angle the flow separation at the rear is more similar to the two-dimensional base flow of the same slant-angle, as evidenced by multiple studies [17, 20, 28].

\subsection{Thesis Outline}

Chapter 1 has provided a brief introduction to the state of bluff body flows and the direction with which this study intends to proceed. A discussion of the background of these flow fields is shown, showing both vehicular shaped models as well as not, with particular interest in this study to the zero-slant angle Ahmed model. In addition, previous advancements in flow control (both active and passive) are highlighted as they relate to the bluff body discussions here.

Chapter 2 is a generic introduction into the underlying numerical methods behind Large Eddy Simulation and Reynolds-Averaged Navier-Stokes, the two different general forms of flow field simulations used throughout this thesis. Specifically in regards to the RANS analysis, both sets of two-equation models used in this thesis are presented $(k-\epsilon R N G$ and $k-\omega S S T)$. 
Chapter 3 focuses on the results of two-dimensional numerical simulations. In this section is the full computational setup including geometry, mesh, and numerical solver. A short numerical validation study is presented. Next the results are presented with specific detail in regards to flow pattern structure and rear pressure coefficient, in addition to overall drag coefficient and Strouhal number and their connection to specific Reynolds number effects. An additional section is included to illustrate the benefits and cons of simultaneous actuator operation.

Chapter 4's main focus is on the three-dimensional simulations. Initially, the RANS setup is presented and analysis against external results are shown in order to verify the reliability on certain aspects such as time-averaged streamlines and average drag coefficient. A second LES setup is analyzed against similar results and then used to evaluate the effectiveness of a suction control scheme. Not only are conclusions based on comparable drag coefficient and streamline data, but additional aspects of the flow field are considered such as Strouhal number.

Chapter 5 will end the thesis with concluding remarks. A brief outlook of future work that could improve upon the claims presented herein is also presented. 


\section{Chapter 2: Turbulence Modeling and Physical Background}

\subsection{Numerical Turbulence Simulation}

Turbulence modeling is a key issue in most computational fluid dynamics simulations. Turbulence consists of three-dimensional chaotic motion that spans a wide range of scales of motion, which increases with Reynolds number. Virtually all engineering applications are turbulent and hence require a turbulence model. The two types of models used in this thesis are described as follows.

\subsubsection{Large Eddy Simulation}

A numerical integration of the full NavierStokes equations that explicitly calculates all scales of turbulent motion is known as direct numerical simulation (DNS). However, DNS can only simulate low to medium Reynolds number turbulence with todays computing power. Large-eddy simulation (LES) uses Kolmogorov's theory of self similarity to explicitly solve for the large eddies in a calculation and implicitly account for the small eddies by using a subgrid-scale model, such as Smagorinski's [29]. In this way, a filtered set of equations can be used throughout the fluid that will cut off such small eddies. The Navier-Stokes equations for an incompressible fluid 
are:

$$
\frac{\partial u_{i}}{\partial t}=-\frac{\partial u_{i} u_{j}}{\partial x_{j}}+X_{i}-\frac{1}{\rho} \frac{\partial p}{\partial x_{i}}+\nu \frac{\partial^{2} u_{i}}{\partial x_{j}^{2}}
$$

where the velocity field $u_{i}$ satisfies the continuity equations

$$
\frac{\partial u_{i}}{\partial x_{i}}=0
$$

In the above equations, $u_{i}$ are flow velocities in the three spatial directions, $X_{i}$ are the components of body force, $\rho$ is the air density, $p$ is the pressure, $\nu$ is the kinematic viscosity of the fluid, $t$ is time, and $x_{i}$ are the spatial coordinates. As noted above, a numerical integration at this point would be known as a DNS calculation, however for LES these equations need to be spatially filtered. This derivation is done by decomposing all dependent variables, for example the velocity field $u_{i}$ into $u_{i}=\tilde{u}_{i}-u_{i}^{\prime \prime}$ where $\tilde{u}_{i}$ is the filter-scale component and $u_{i}^{\prime \prime}$ is the subgrid scale. Applying the filtering procedure to the above equations leads to the following equation, which will govern large eddies:

$$
\frac{\partial \tilde{u}_{i}}{\partial t}=-\frac{\partial \tilde{u}_{i} \tilde{u}_{j}}{\partial x_{j}}-\frac{\partial \tau_{i j}}{\partial x_{j}}+X_{i}-\frac{1}{\rho_{0}} \frac{\partial \tilde{p}}{\partial x_{i}}+\nu \frac{\partial^{2} \tilde{u}_{i}}{\partial x_{j}^{2}}
$$

where the subgrid-scale stress is defined by $\tau_{i j}[30]$.

\subsubsection{Reynolds-Averaged Simulation}

Reynolds-Averaged Navier-Stokes (RANS) equations are based on the knowledge that a time dependent signal can be decomposed based on the fluctuations of a given point. For example:

$$
\phi\left(x_{i}, t\right)=\bar{\phi}\left(x_{i}\right)+\phi^{\prime}\left(x_{i}, t\right)
$$


where $\bar{\phi}\left(x_{i}\right)$ is the mean part, and $\phi^{\prime}\left(x_{i}, t\right)$ is the fluctuating part of the turbulence field. Similar to the LES filtering, decomposition can be applied to the Navier-Stokes equations (i.e. $u_{i}$ into $u_{i}=\bar{u}_{i}-u_{i}^{\prime}$ ) resulting in the form known as RANS equations:

$$
\rho \bar{u}_{j} \frac{\partial \bar{u}_{i}}{\partial x_{j}}=-\frac{\partial \bar{p}}{\partial x_{i}}+\mu \frac{\partial \frac{\partial \bar{u}_{i}}{\partial x_{j}}}{\partial x_{j}}-\rho \frac{\partial u_{i}^{\prime} u_{j}^{\prime}}{\partial x_{j}}+\rho f
$$

The only new term expressed here is the $u_{i}^{\prime} u_{j}^{\prime}$ known as the Reynold's stress tensor, which accounts for the information lost due to averaging. This term is an unknown and presents a closure problem numerically, which algorithms known as turbulence models seek to close by using correlations of known quantities of the flow field to approximate it [21].

The two different turbulence models used in the thesis are categorized as linear eddy viscosity models; two-equation models. The turbulent properties of the flow are represented by two extra transport equations, which by definition make up the twoequation models. History effects such as convection and diffusion of turbulent energy can be accounted for using these two extra transport equations. One of the transported variables is the turbulent kinetic energy, $k$. The other transported variable is dependent on which two-equation model is in use.

For the $k-\epsilon$ Renormalization Group (RNG) model, the Navier-Stokes equations are renormalized to account for the effects of smaller scales of motion. The eddy viscosity is determined from a single turbulence length scale in the standard $k-\epsilon$ model, so the calculated turbulent diffusion is that which occurs only at that specific scale, whereas in reality all scales of motion contribute to turbulent diffusion. A turbulence model derived from the $k-\epsilon$ standard results in a modified form of the equations, shown below, which attempt to account for the different scales of motion through changes to the production term. 


$$
\begin{gathered}
\frac{\partial}{\partial t}(\rho k)+\frac{\partial}{\partial x_{i}}\left(\rho k u_{i}\right)=\frac{\partial}{\partial x_{j}}\left[\left(\mu+\frac{\mu_{t}}{\sigma_{k}}\right) \frac{\partial k}{\partial x_{j}}\right]+P_{k}-\rho \epsilon \\
\frac{\partial}{\partial t}(\rho \epsilon)+\frac{\partial}{\partial x_{i}}\left(\rho \epsilon u_{i}\right)=\frac{\partial}{\partial x_{j}}\left[\left(\mu+\frac{\mu_{t}}{\sigma_{\epsilon}}\right) \frac{\partial \epsilon}{\partial x_{j}}\right]+C_{1 \epsilon} \frac{\epsilon}{k} P_{k}-C_{2 \epsilon}^{*} \rho \frac{\epsilon^{2}}{k}
\end{gathered}
$$

where $C_{2 \epsilon}^{*}=C_{2 \epsilon}+\frac{C_{\mu} \eta^{3}\left(1-\eta / \eta_{0}\right)}{1+\beta \eta^{3}}$ and $\eta=S k / \epsilon$ and $S=\left(2 S_{i j} S_{i j}\right)^{1 / 2}$

with the turbulent viscosity being calculated in the same manner as with the standard k-epsilon model[31].

The $k-\omega$ SST turbulence model uses a $k-\omega$ formulation in the inner parts of the boundary layer, making the model directly usable down to the wall through the viscous sub-layer without any extra damping functions. The SST formulation also switches to a $k-\epsilon$ behaviour in the free-stream and thereby avoids the common $k-\omega$ problem that the model is too sensitive to the inlet free-stream turbulence properties. Equations for the turbulent kinetic energy and specific dissipation rate $(\omega)$ are shown below.

$$
\begin{gathered}
\frac{\partial k}{\partial t}+U_{j} \frac{\partial k}{\partial x_{j}}=P_{k}-\beta^{*} k \omega+\frac{\partial}{\partial x_{j}}\left[\left(\nu+\sigma_{k} \nu_{T}\right) \frac{\partial k}{\partial x_{j}}\right] \\
\frac{\partial \omega}{\partial t}+U_{j} \frac{\partial \omega}{\partial x_{j}}=\alpha S^{2}-\beta \omega^{2}+\frac{\partial}{\partial x_{j}}\left[\left(\nu+\sigma_{\omega} \nu_{T}\right) \frac{\partial \omega}{\partial x_{j}}\right]+2\left(1-F_{1}\right) \sigma_{\omega 2} \frac{1}{\omega} \frac{\partial k}{\partial x_{i}} \frac{\partial \omega}{\partial x_{i}}
\end{gathered}
$$




\section{Chapter 3: Two-Dimensional Experiments}

\subsection{Simulation Setup}

Throughout the two-dimensional simulations and analysis the ANSYS Workbench suite is utilized, including the in-software packages for geometry creation, mesh generation, computational solver, and post processing. The parametric study consists of Reynolds numbers between one and four million, in increments of approximately 300000 , and a range of equivalent actuator power from $0.5 \cdot U_{\infty} t o 2.0 \cdot U_{\infty}$.

\subsubsection{Geometry Creation \& Mesh Generation}

The size and shape of the bluff body studied in this thesis is almost identical to the original model introduced by Ahmed [11]. In particular, the supporting struts have been removed and the slanted rear end is replaced with a square-back. It has a length of $1.044 \mathrm{~m}$, a height of $0.288 \mathrm{~m}$, a distance above the ground of $0.05 \mathrm{~m}$ and edges rounded at the front with a radius of $0.1 \mathrm{~m}$, as shown in Figure 3.1. The computational domain extends one body length upstream of the model, five lengths downstream and three body lengths up. The projected area of the Ahmed body in the mainstream direction is $0.112 \mathrm{~m}^{2}$ corresponding to a blockage ratio of approximately $5 \%$. 


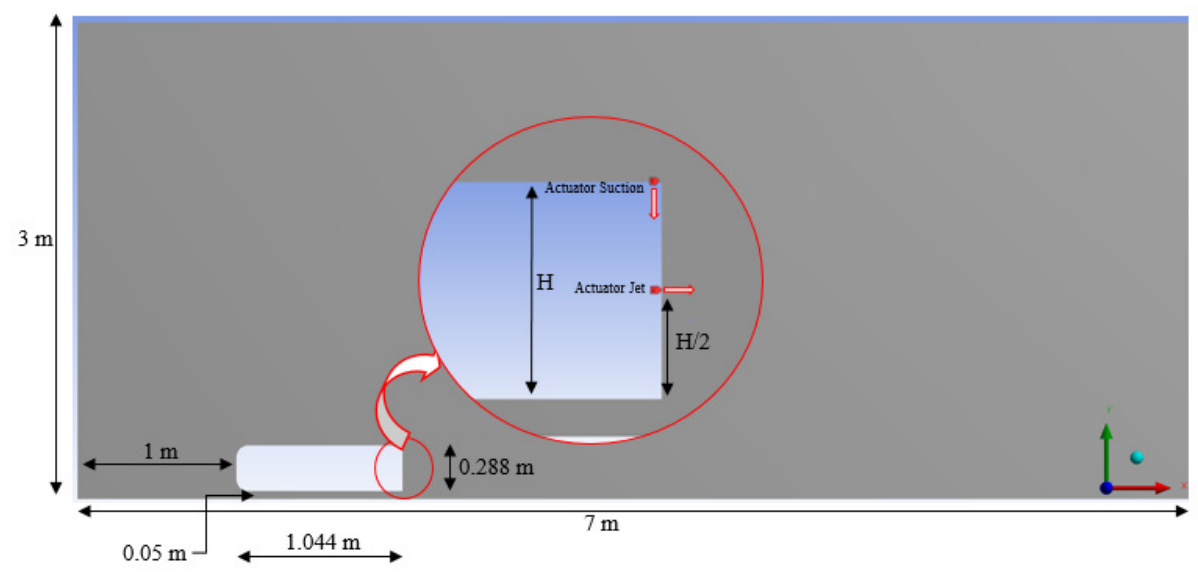

Figure 3.1: Numerical boundary conditions and general domain dimensions

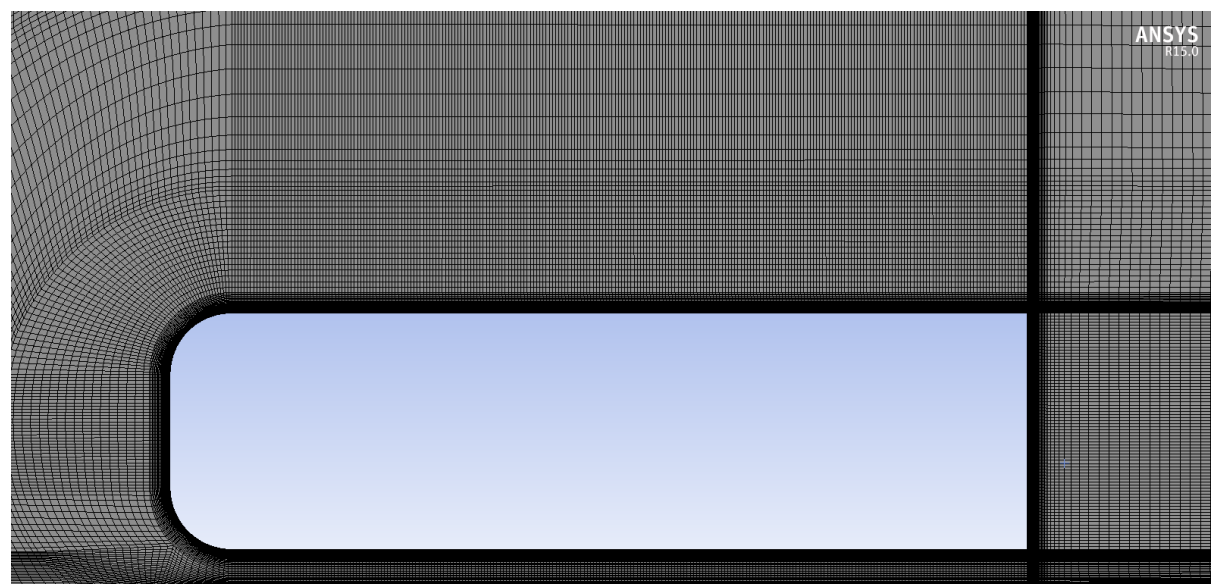

Figure 3.2: Near-body structured mesh of computational domain

Figure 3.2 shows a small snapshot of the near-body mesh configuration. The nonuniform structured grid is constructed, with the near wall region using smaller grid sizes to control the $\mathrm{y}+$ at 30 . The total cell count stands at approximately 153000 . 


\subsubsection{Physical Models}

FLUENT is a commercial flow solver package developed by ANSYS and included in the ANSYS Workbench Suite. The software contains the broad physical modeling capabilities needed to model flow, turbulence, heat transfer, and reactions for countless fluid mechanical applications.

\section{Turbulence Models and Discretization}

Of the numerous turbulence models contained in the FLUENT package, this two-dimensional thesis section utilizes the $\mathrm{k}-\epsilon$ turbulence model, specifically ReNormalization group. When using a turbulence model instead of a direct Navier Stokes solver such as DNS or LES, absolute errors are introduced into the solution in addition to limiting the overall flow characteristics accuracy. For this reason, higher emphasis is placed on flow characteristic trends as opposed to raw data values. In order to enhance the accuracy of the solution, an enhanced non-equilibrium wall treatment is utilized, which has been proven to perform well in adverse pressure gradient environments. Additionally, the ideal gas formulation for density is employed as required by the boundary conditions. The PISO scheme is used in regards to pressure-velocity coupling, however skewness-neighbor coupling is disabled. Momentum discretization along with both turbulence model variables (k and $\epsilon$ ) use the central scheme QUICK, but density and energy equations use second order upwind. Pressure discretization utilizes the PRESTO algorithm and the transient formulation is in first order implicit. In the case of the LES simulation, skewness-neighbor cou-

pling is re-enabled and pressure is discretized using the Standard algorithm rather than PRESTO. 


\section{Boundary Conditions}

In regards to boundary treatment in the context of FLUENT, each boundary is set to a specific condition. The front boundary is modeled as a velocity inlet with turbulence specification of 0.25 intensity and viscosity ratio of 10 . The bluff-body itself and ground plane are both considered as no-slip walls, with the ground plane moving at the same velocity as the inlet. For the rear boundary a pressure outlet at the same turbulence specification as the inlet is prescribed with zero gage pressure. Finally, the upper boundary is designated as a far-field pressure condition at the same Mach number and turbulence specifications as the incoming flow.

For active control, two different actuators have been utilized and are depicted in Figure 3.1. These actuators have non-dimensional thicknesses of $3 \%$ of body height. The first is the horizontal jet located at the center of the rear back model body, and the second is a suction jet placed at the top corner edge of the model. In terms of Fluent implementation, these two actuators are implemented numerically as velocity boundary conditions. Specifically velocity inlets placed directly on the body, with flow in the positive $\mathrm{x}$-direction and negative $\mathrm{y}$-direction for the respective jet and suction actuators.

\subsection{Validation Study}

In order to verify that the numerical model was going to accurately represent relevant flow field physics, a comparison to real world experiments had to be done. In this case, the $25^{\circ}$ slant angle model was used due to both data availability as well as the increased complexity of the flow field. Figure 3.3 is a direct comparison of the region of attachment / detachment and recirculation at the rear of the Ahmed 


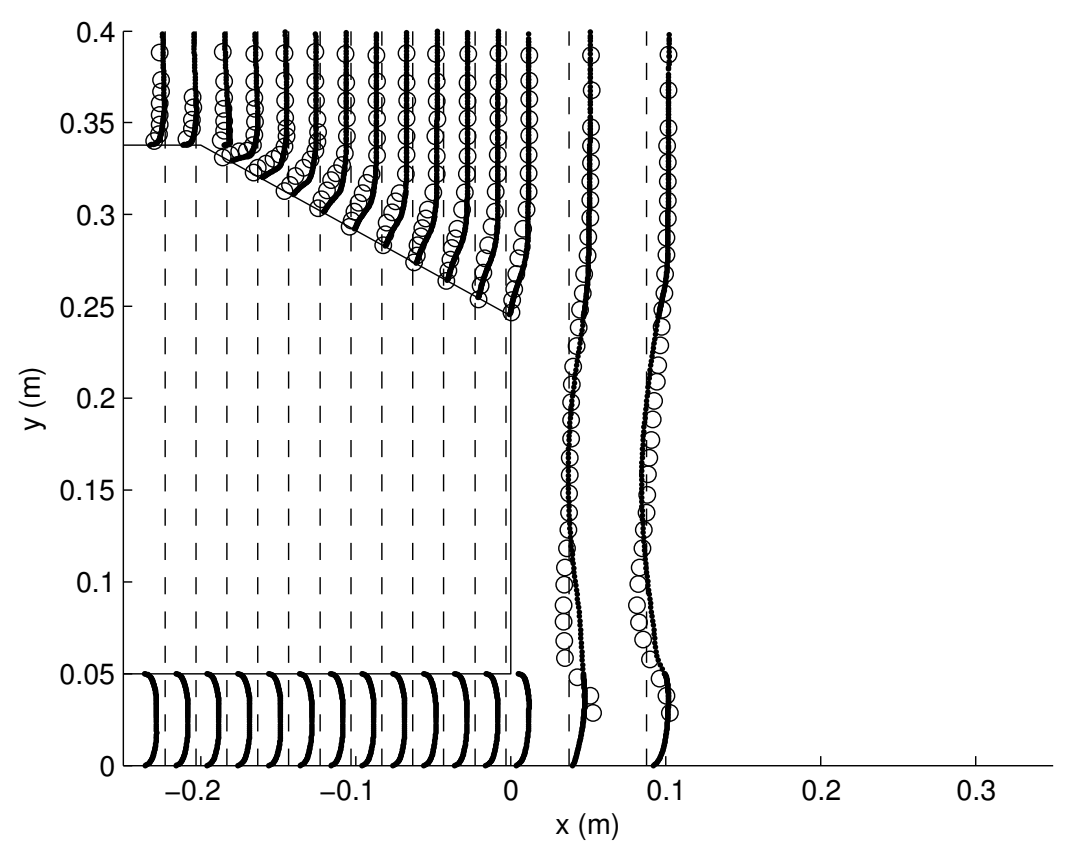

Figure 3.3: Verification of numerical model (o Experimental [15] , • k- $\epsilon$ RNG )

model. The comparison is done by showcasing relative axial velocity measurements at several vertical locations along the body, measured along the plane of symmetry in the case of the experiments. From figure 3.3 a very small embedded recirculation region on the upper part of the slant surface is captured by the numerical model, as well as the second, larger recirculation region beyond the body itself.

In addition to the comparison above, a second study was performed using the more accurate Large Eddy Simulation model. Typically the LES model is barred from use in cases of only two dimensions, because the energy-transfer mechanism cannot be reproduced by $2 \mathrm{D}$ computations since it is fundamentally due to the vortex-stretching mechanism, which is essentially a 3D phenomenon. However, following the guidelines referenced by L. Bruno [33] it is possible to generate a numerical result using LES that 
coincides with the 3D representation. The author takes the 2D LES approach and uses the case study of the bluff deck model to run an extensive parametric analysis. They discovered which schemes are the most accurate in reproducing known flow physics on their model specifically in addition to what value to use for Cs (the subgrid scale model constant). With this combination of parameters and schemes their model takes into account the effects of physical phenomena (vortex stretching, longitudinal vortices) that are generally observed in the case of 3D separated flows. Thereby showing that the overall accuracy of the optimized 2D model is comparable to the 3D simulations. Figure 3.4 shows the comparison of flow fields between this compensated 2D LES and the turbulence model used throughout the remainder of the $2 \mathrm{D}$ study. As can clearly be seen, the alternating shedding vortices appear in both simulations despite the difference in models. Figure 3.5 shows additional data from the model comparison, mainly drag coefficient, however a rough comparison of the two shedding frequencies can also be seen. Since the two numerical models utilize different methods for near-wall treatment, it is not surprising that their drag coefficients differ as well. However the main flow physics that the $\mathrm{k}-\epsilon$ turbulence model attempts to capture as well as LES are confirmed here by comparison of the respective Strouhal numbers (0.234 with k- $\epsilon$ vs 0.240 with LES).

\subsection{Numerical Results and Flow Analysis}

The research done here focuses on the flow pattern changes at the rear end of the bluff body in the near wake flow. In an attempt to discover how these actuators affect this set of flow patterns, a wide range of conditions were tested, including employing each actuator separately at varying fractions of incoming velocity and at 


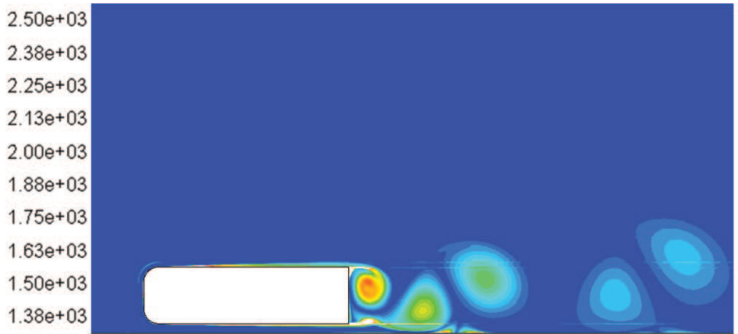

(a)

$1.13 e+03$

$1.00 e+03$

$8.75 e+02$

$7.50 \mathrm{e}+02$

$6.25 \mathrm{e}+02$

$5.00 \mathrm{e}+02$

$3.75 e+02$

$2.50 \mathrm{e}+02$

$1.25 \mathrm{e}+02$

$0.00 \mathrm{e}+00$

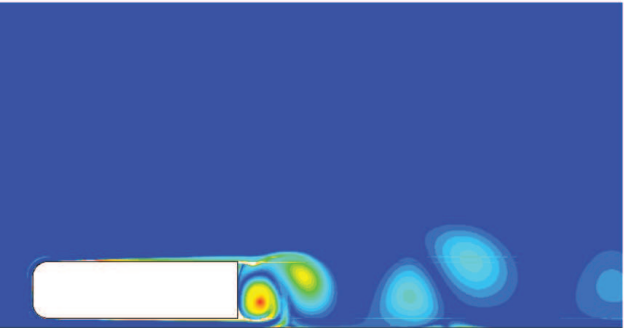

(b)

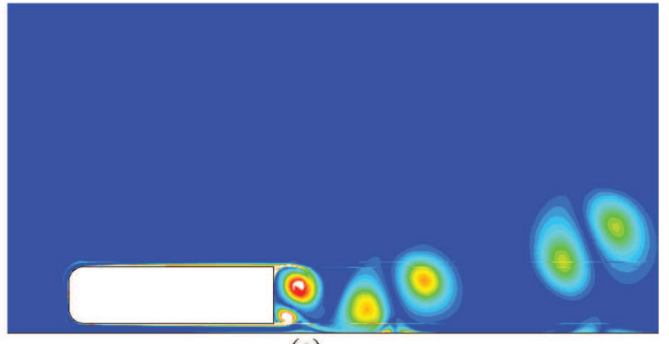

(c)

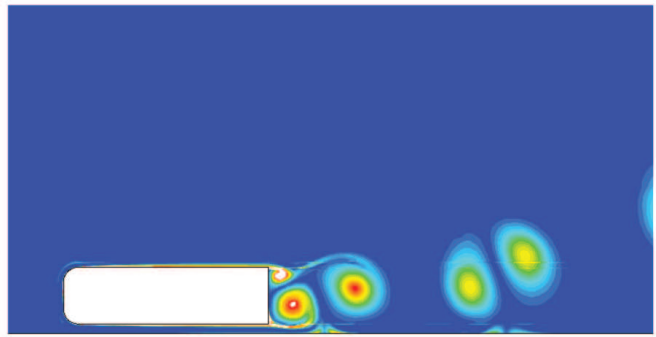

(d)

Figure 3.4: Instantaneous vorticity contours of base case in $60 \mathrm{~m} / \mathrm{s}$ flow, (a-b) $k-\epsilon$ RNG (c-d) LES

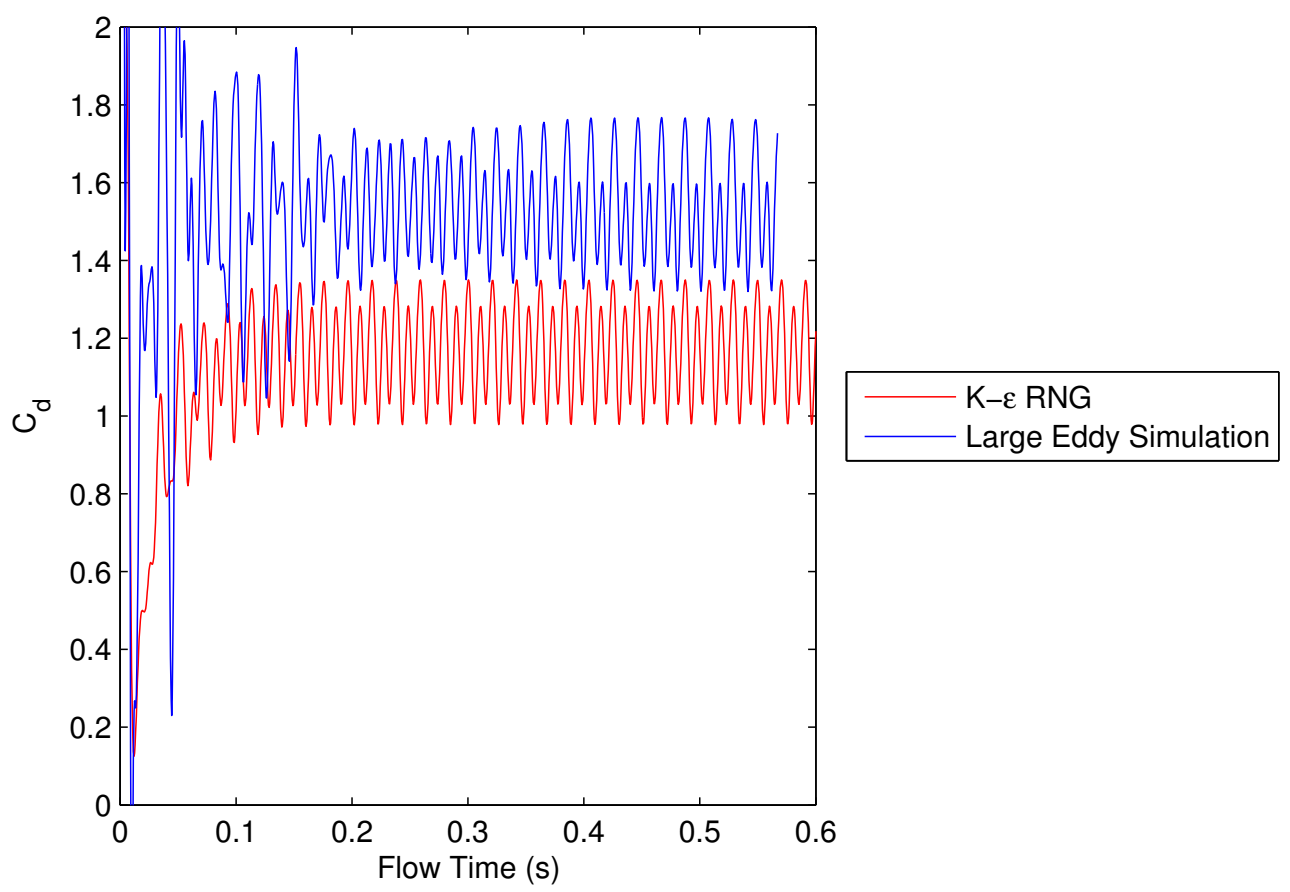

Figure 3.5: Transient drag comparison between the two different methods 
varying Reynolds numbers. Limitations here include a lack of analysis of the required power, as this study is solely focused on the pattern the actuators enforce on the flow. To keep the data consistent each actuator is only ever run on its own, never simultaneously, and velocity of the velocity inlet boundary conditions are used as power required guidelines throughout the experiment. These two actuators are powered by velocities ranging from half the free-stream to double the free-stream, and the body sees incoming Reynolds number flow from 1 to 4 million. All simulations were run in the transient state with a time-step of 0.0005 seconds, with typical residuals of the Navier Stokes equations at 10E-6 for confidence in the solution. When determining the two quantities of interest, drag coefficient and Strouhal number, data from several periods of shedding was analyzed. For $C_{d}$ a simple mean calculation was used, but for St the MATLAB FFT (Fast Fourier Transform) function was used to obtain the dominant shedding frequency. An instance of these calculations can be seen in Figure 3.6 .

\subsubsection{Flow Pattern Structure}

For the square-back bluff body without any fluid control, the flow separates at the back and is dominated by counter-rotating structures shown in Figure 3.7, which represents flow patterns by showing various vorticity contours. Experimental and numerical studies confirm this two-dimensional behavior of the detached near-wall flow $[6,10,20,23,34]$.

By utilizing the suction actuator at the top end of the model, this flow pattern is eventually altered into the completely different steady-state configuration shown in Figure 3.8. Since both figures are generated at the same contour level it is easy to see 

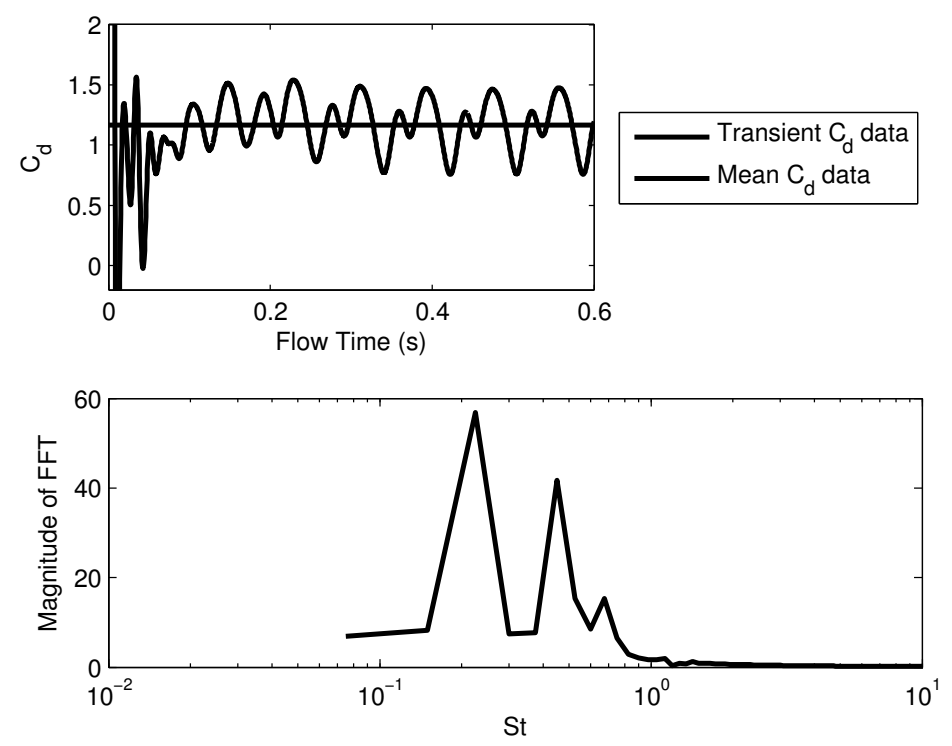

Figure 3.6: (Upper) Typical periodic drag coefficient (Lower) MATLAB fast Fourier transform of drag coefficient for Strouhal number (Suction actuator at $7.5 \mathrm{~m} / \mathrm{s}$ in 15 $\mathrm{m} / \mathrm{s}$ flow)

that, although a slight increase in vorticity near the suction actuator is perceived, the bulk flow pattern has ceased shedding altogether. In a similar fashion to the suction actuation, the blowing jet is effective at altering the flow pattern into a comparable but different steady-state configuration at a smaller Reynolds number than the suctioncontrolled flow. This is shown in the bottom half of Figure 3.8, again with the use of vorticity contours.

As the goal of this endeavor was in part to control the wake vortices, we will see in section 3.3.3 how the effects from each actuators' steady-state results effect the overall drag coefficient. 


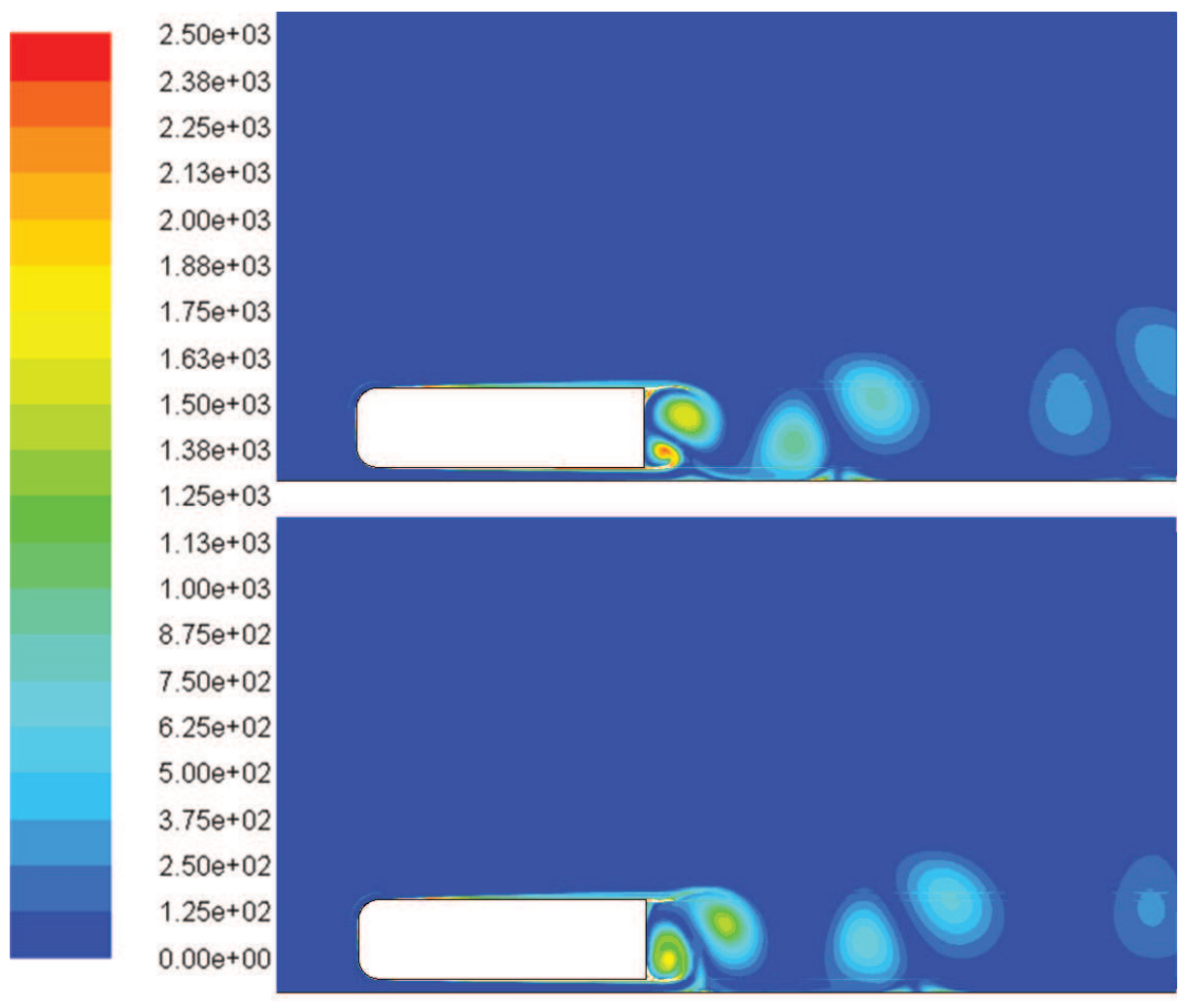

Figure 3.7: Shedding of base case at Reynolds number 2.67 million shown with vorticity contours, $\Delta \mathrm{t}=0.02 \mathrm{~s}$ 


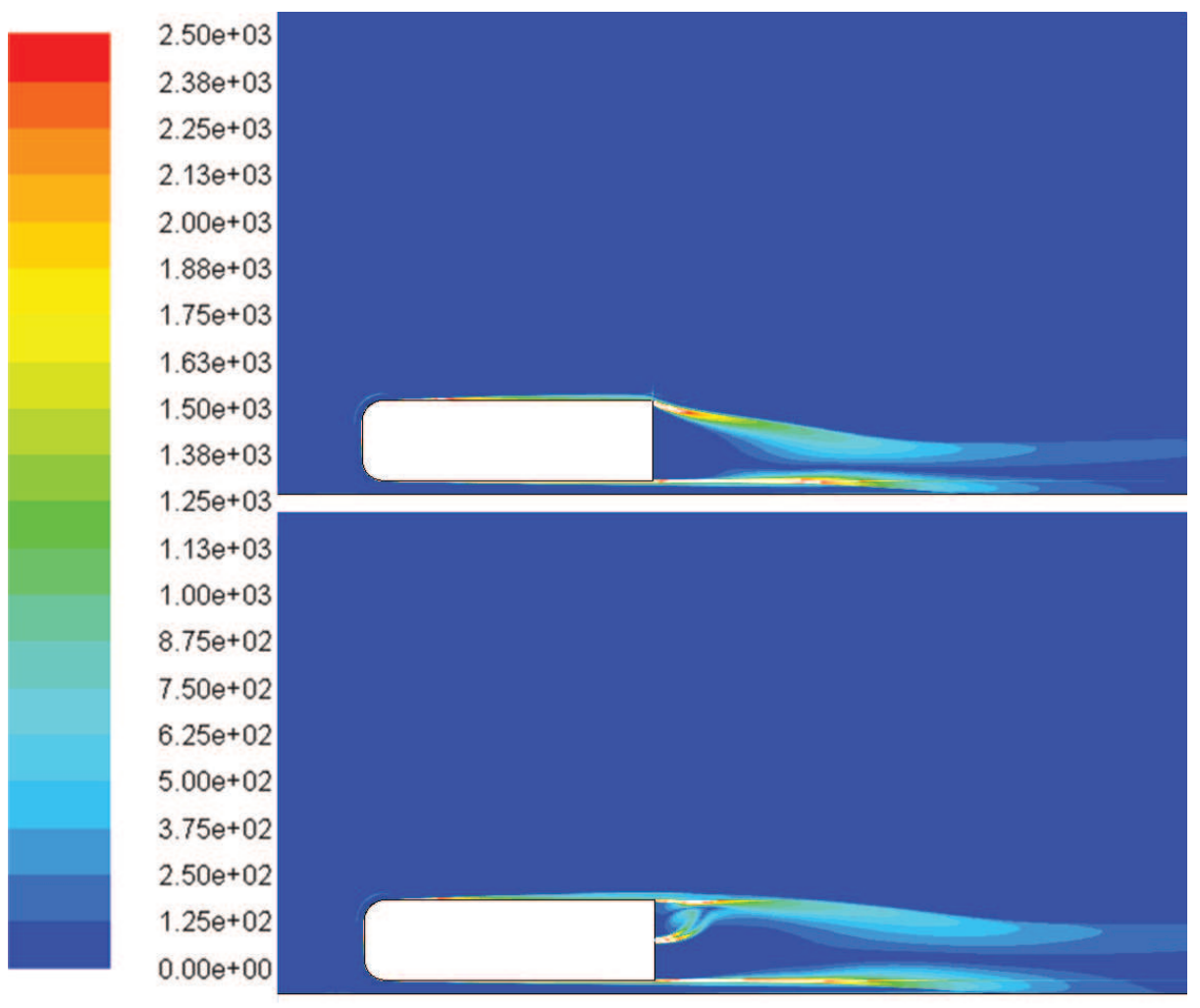

Figure 3.8: Vorticity contours of steady state (top) suction actuation at $82.5 \mathrm{~m} / \mathrm{s}$ in $55 \mathrm{~m} / \mathrm{s}$ flow (bottom) blowing actuation at $45 \mathrm{~m} / \mathrm{s}$ in $45 \mathrm{~m} / \mathrm{s}$ flow 


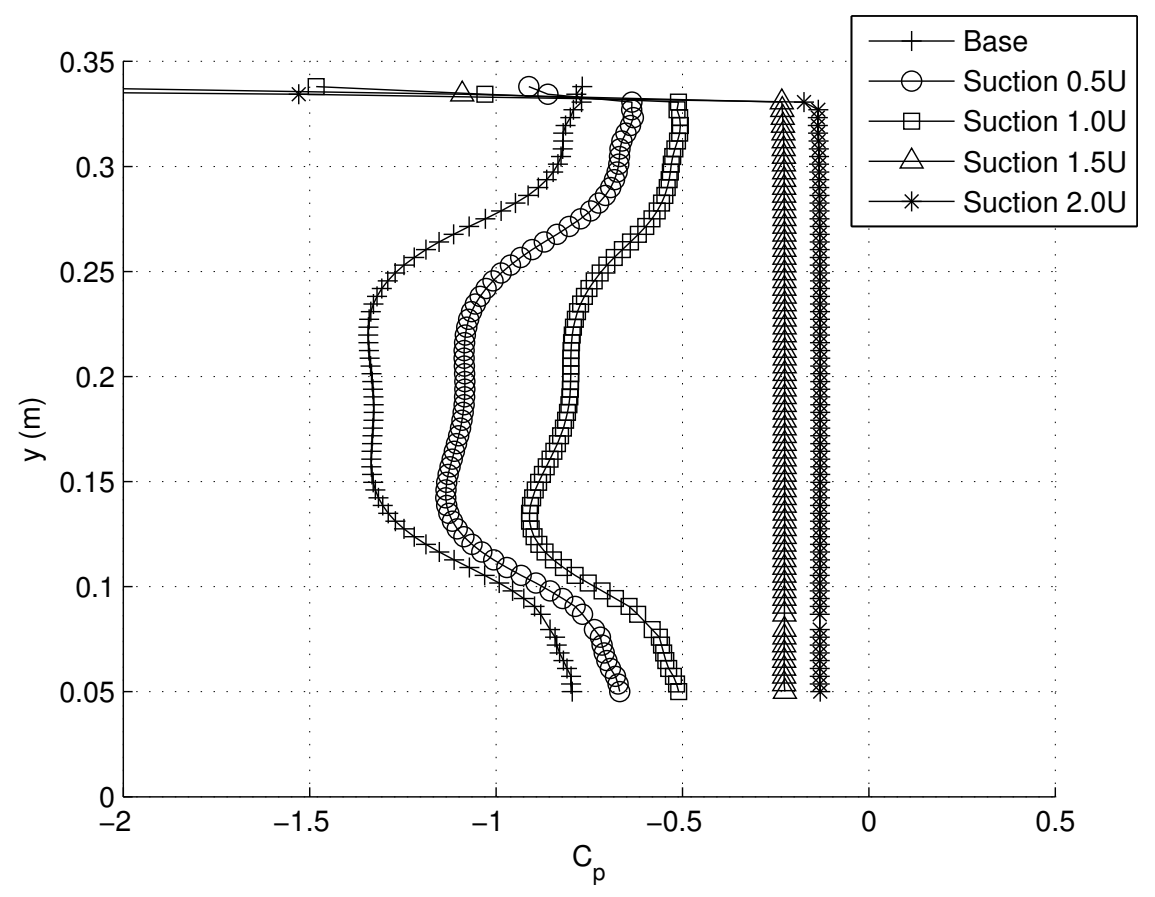

Figure 3.9: Example plot of pressure coefficient with suction actuator at $\operatorname{Re}=3.66$ million

\subsubsection{Rear-Model Pressure Coefficient}

In addition to flow topology it is also important to look at how these controls effect the back-pressure of the model, specifically the pressure coefficient. Figure 3.9 depicts the average pressure coefficient up the rear of the model of a few notable suction-actuated cases. It is clear from the trends shown in the figure that the suction actuator facilitates a relatively uniform increase in back-pressure, which is a known cause of drops in pressure-based wake drag.

However, when discussing jet-actuated cases instead of a uniform increase in backpressure the jet has a stronger more localized effect in the area of the jet that drops off as the air reaches farther from it. This relationship is shown in Figure 3.10 for a few specific cases (additional cases located in Appendix A). The sudden change 


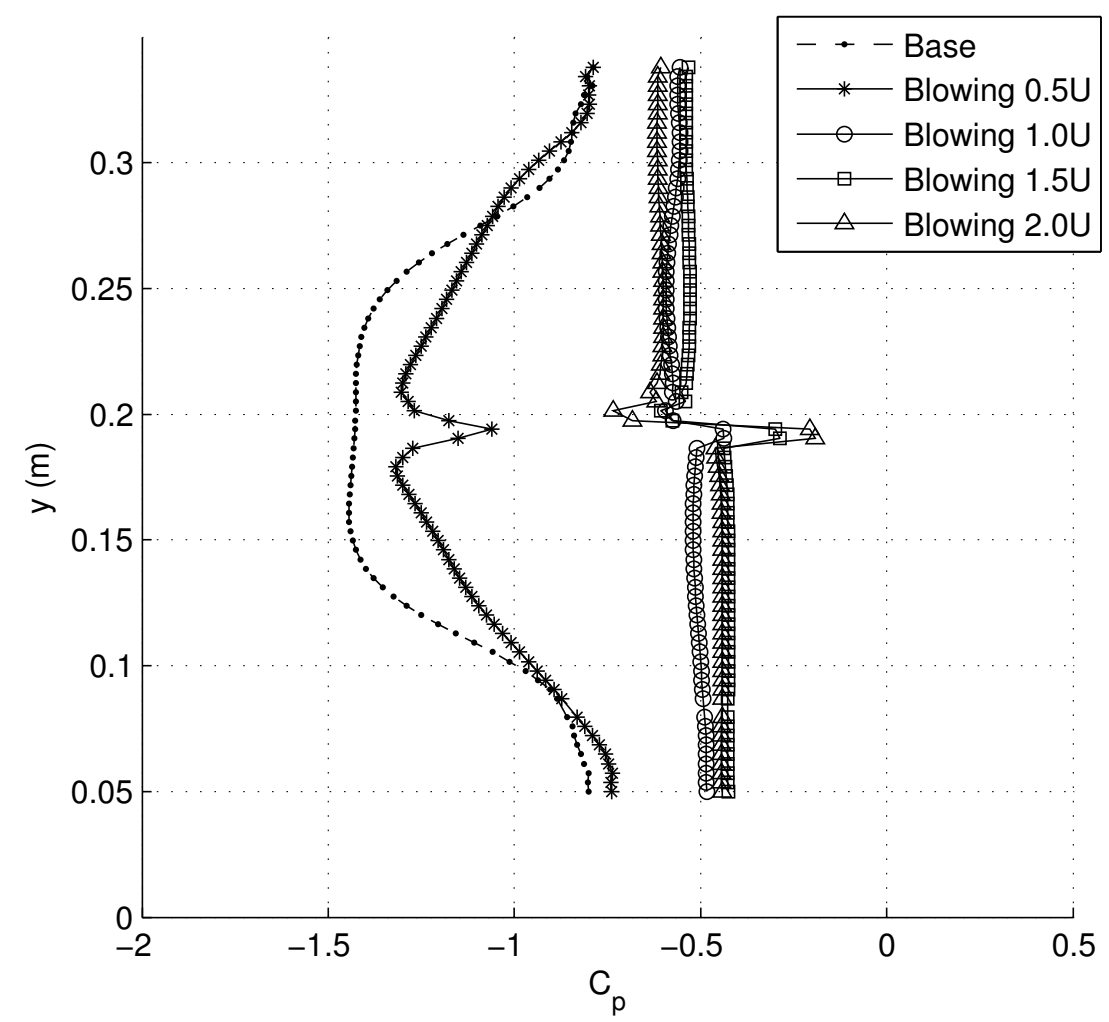

Figure 3.10: Example plot of pressure coefficient with blowing actuator at $\operatorname{Re}=3$ million

in shape of the pressure coefficient trend coincides precisely with when the actuator ceases the shedding.

\section{Reynolds Number Effect}

Before the introduction of Strouhal number and drag coefficient data, a short discussion on the effect of Reynolds Number on the pressure coefficient and ultimately drag coefficient is presented. Figure 3.11 shows the pressure coefficient at the rear of the non-actuated base model for various Reynolds numbers. A trend much similar to that of the suction controlled cases emerges, as discussed later in Section 3.3.3. As 


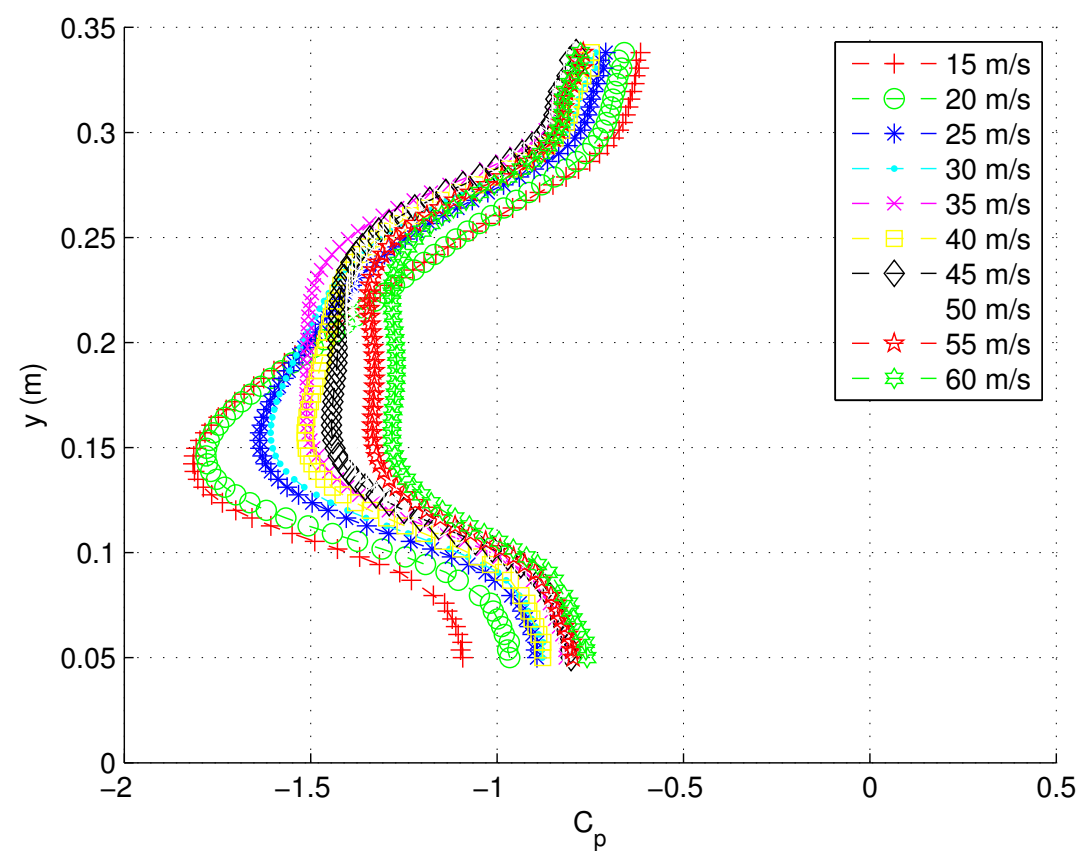

Figure 3.11: Pressure coefficient of base case at various Reynolds numbers

Reynolds number is increased the pressure coefficient increases as well, in a relatively uniform way much like that of the suction actuated cases albeit at a lesser scale, as is expected.

\subsubsection{Effects of Control Schemes on Strouhal Number and Drag Coefficient}

Both the flow pattern data from section 3.3.1 and the pressure coefficient data from 3.3.2 indicate that these respective controls will in fact reduce the drag coefficient of the model, but the interesting discoveries lie in which actuator is more effective. The complete datasets are shown in Table 3.1 for the suction actuator and Table 3.2 for the jet actuator. 


\begin{tabular}{ccccccccccc} 
& \multicolumn{1}{c}{ Base (no control) } & \multicolumn{2}{c}{$0.5 \cdot U_{\infty}$} & \multicolumn{2}{c}{$1.0 \cdot U_{\infty}$} & \multicolumn{2}{c}{$1.5 \cdot U_{\infty}$} & \multicolumn{2}{c}{$2.0 \cdot U_{\infty}$} \\
\hline Reynolds Number & $C_{d}$ & $\mathrm{St}$ & $C_{d}$ & $\mathrm{St}$ & $C_{d}$ & $\mathrm{St}$ & $C_{d}$ & $\mathrm{St}$ & $C_{d}$ & St \\
\hline $1.00 \mathrm{E}+06$ & 1.409 & 0.221 & 1.167 & 0.234 & 0.966 & 0.246 & 0.772 & 0.254 & 0.559 & 0.261 \\
$1.33 \mathrm{E}+06$ & 1.365 & 0.225 & 1.154 & 0.236 & 0.956 & 0.246 & 0.760 & 0.255 & 0.550 & 0.262 \\
$1.66 \mathrm{E}+06$ & 1.343 & 0.226 & 1.144 & 0.235 & 0.948 & 0.245 & 0.743 & 0.253 & 0.517 & 0.265 \\
$2.00 \mathrm{E}+06$ & 1.345 & 0.229 & 1.137 & 0.237 & 0.931 & 0.246 & 0.723 & 0.256 & 0.480 & 0.263 \\
$2.33 \mathrm{E}+06$ & 1.318 & 0.229 & 1.114 & 0.239 & 0.910 & 0.249 & 0.698 & 0.253 & 0.471 & 0.265 \\
$2.66 \mathrm{E}+06$ & 1.306 & 0.229 & 1.089 & 0.236 & 0.882 & 0.248 & 0.669 & 0.257 & 0.450 & 0.267 \\
$3.00 \mathrm{E}+06$ & 1.276 & 0.229 & 1.061 & 0.242 & 0.848 & 0.251 & 0.631 & 0.261 & 0.404 & 0.272 \\
$3.33 \mathrm{E}+06$ & 1.246 & 0.230 & 1.028 & 0.240 & 0.807 & 0.250 & 0.585 & 0.262 & 0.125 & \\
$3.66 \mathrm{E}+06$ & 1.212 & 0.233 & 0.988 & 0.244 & 0.757 & 0.255 & 0.222 & & 0.128 & \\
$4.00 \mathrm{E}+06$ & 1.172 & 0.234 & 0.937 & 0.240 & 0.685 & 0.259 & 0.221 & & 0.132 & \\
\hline
\end{tabular}

Table 3.1: Data from suction simulations on square-back Ahmed body (absence of St entry indicates shedding stoppage)

\begin{tabular}{ccccccccccc} 
& \multicolumn{2}{c}{ Base (no control) } & \multicolumn{2}{c}{$0.5 \cdot U_{\infty}$} & \multicolumn{2}{c}{$1.0 \cdot U_{\infty}$} & \multicolumn{2}{c}{$1.5 \cdot U_{\infty}$} & \multicolumn{2}{c}{$2.0 \cdot U_{\infty}$} \\
\hline Reynolds Number & $C_{d}$ & $\mathrm{St}$ & $C_{d}$ & $\mathrm{St}$ & $C_{d}$ & $\mathrm{St}$ & $C_{d}$ & $\mathrm{St}$ & $C_{d}$ & $\mathrm{St}$ \\
\hline $1.00 \mathrm{E}+06$ & 1.409 & 0.221 & 1.474 & 0.236 & 1.395 & 0.251 & 1.153 & 0.253 & 0.591 & \\
$1.33 \mathrm{E}+06$ & 1.365 & 0.225 & 1.410 & 0.240 & 1.323 & 0.253 & 1.196 & 0.255 & 0.578 & \\
$1.66 \mathrm{E}+06$ & 1.343 & 0.226 & 1.330 & 0.240 & 1.235 & 0.253 & 1.174 & 0.253 & 0.571 & \\
$2.00 \mathrm{E}+06$ & 1.345 & 0.229 & 1.274 & 0.240 & 1.159 & 0.253 & 0.522 & & 0.560 \\
$2.33 \mathrm{E}+06$ & 1.318 & 0.229 & 1.225 & 0.242 & 1.099 & 0.249 & 0.514 & & 0.556 \\
$2.66 \mathrm{E}+06$ & 1.306 & 0.229 & 1.194 & 0.240 & 1.001 & 0.253 & 0.509 & & 0.734 \\
$3.00 \mathrm{E}+06$ & 1.276 & 0.229 & 1.139 & 0.242 & 0.487 & & 0.507 & & 0.552 \\
$3.33 \mathrm{E}+06$ & 1.246 & 0.230 & 1.089 & 0.245 & 0.477 & & 0.546 & & 0.551 \\
$3.66 \mathrm{E}+06$ & 1.212 & 0.233 & 1.059 & 0.244 & 0.476 & & 0.505 & & 0.551 \\
$4.00 \mathrm{E}+06$ & 1.172 & 0.234 & 1.009 & 0.246 & 0.476 & & 0.505 & & 0.551 \\
\hline
\end{tabular}

Table 3.2: Data from blowing simulations on square-back Ahmed body (absence of St entry indicates shedding stoppage) 


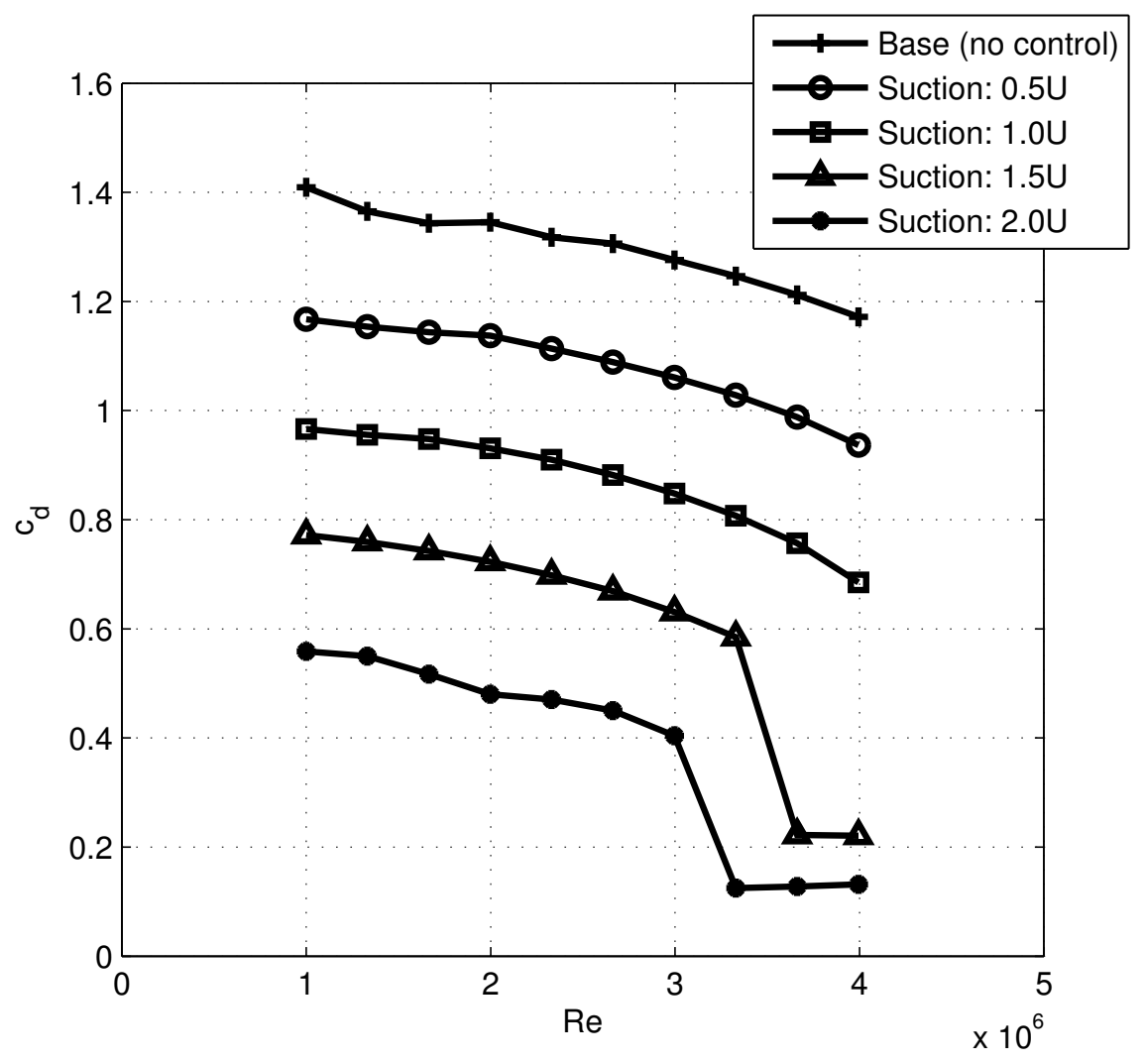

Figure 3.12: Graphical depiction of drag coefficient data from Table 3.1

The same data is represented again in graphical form with Figures 3.12 and 3.13 showing suction-actuated drag coefficient and Strouhal number respectively, in addition to Figures 3.14 and 3.15 with their respective blowing jet counterparts.

As can be easily seen from Figures 3.12 and $3.1498 \%$ of all cases tested (both suction and blowing) saw a reduction in drag. The Reynolds number effect discussed in section 3.3.2 is present again in these figures. From section 3.3.2 it is noted that the alterations to pressure coefficient due to increases in Reynolds number resemble those caused by the suction actuator, albeit at a lesser magnitude. This leads to the assumption that a decrease in drag similar to that of the suction actuators will accompany the corresponding changes in pressure coefficient, which is exactly what 


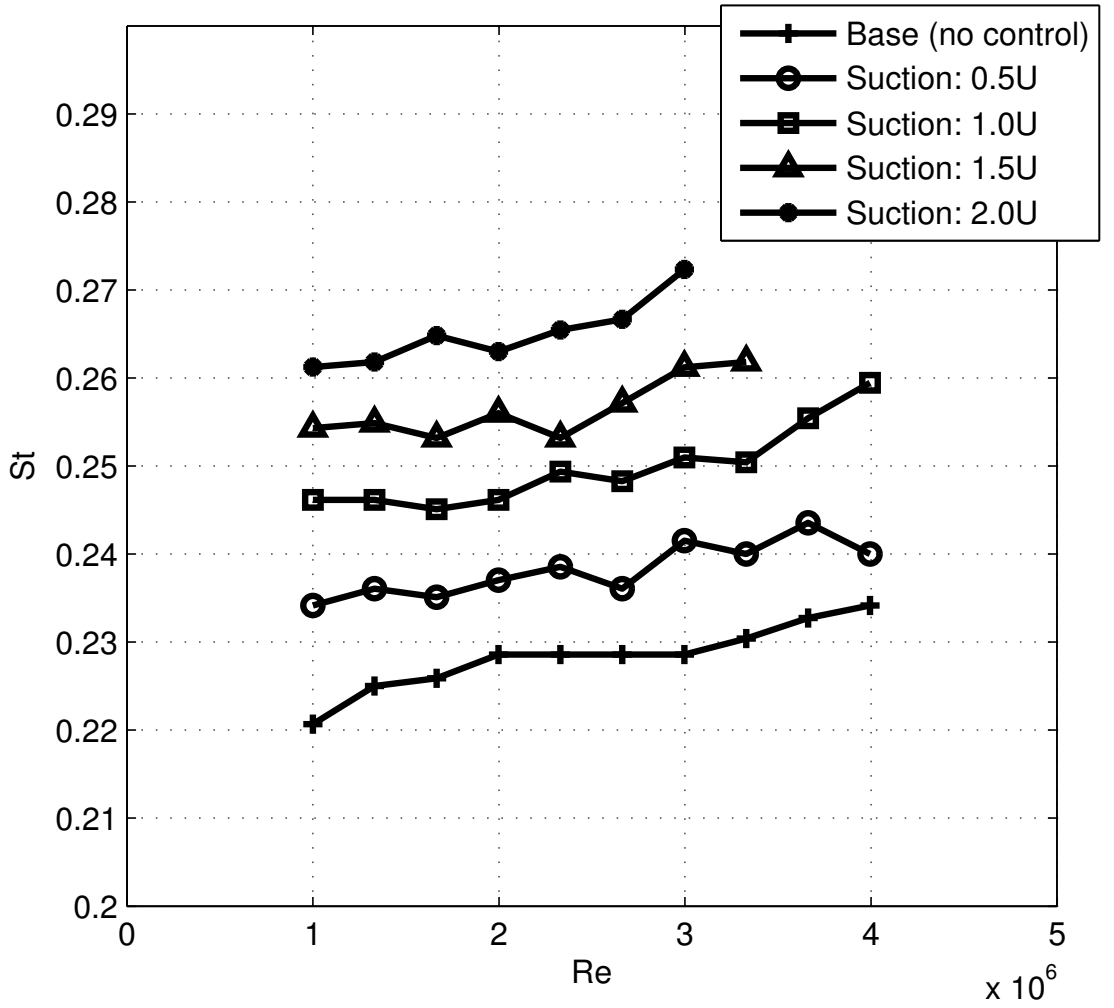

Figure 3.13: Graphical depiction of Strouhal number data from Table 3.1 


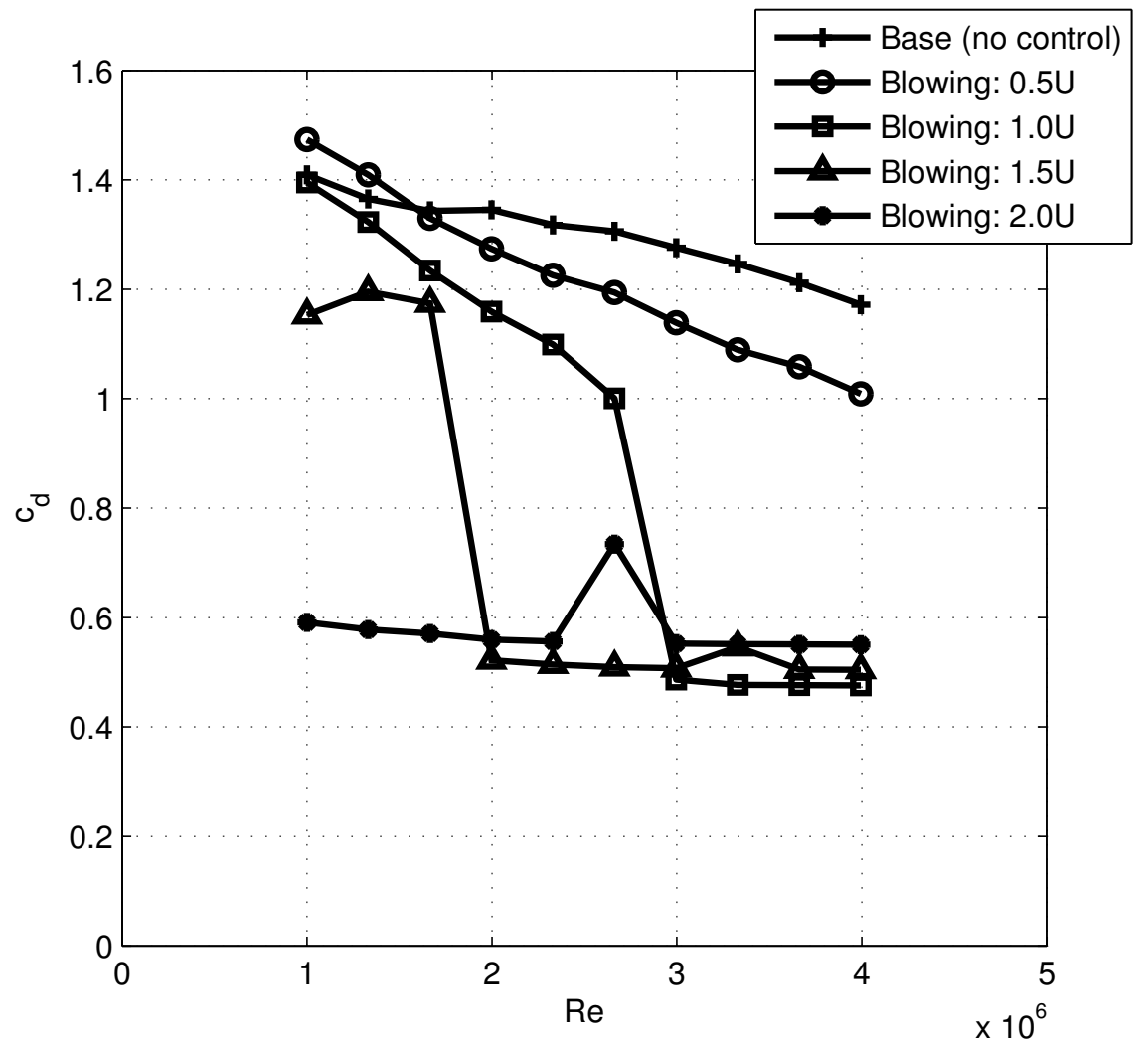

Figure 3.14: Graphical depiction of drag coefficient data from Table 3.2 


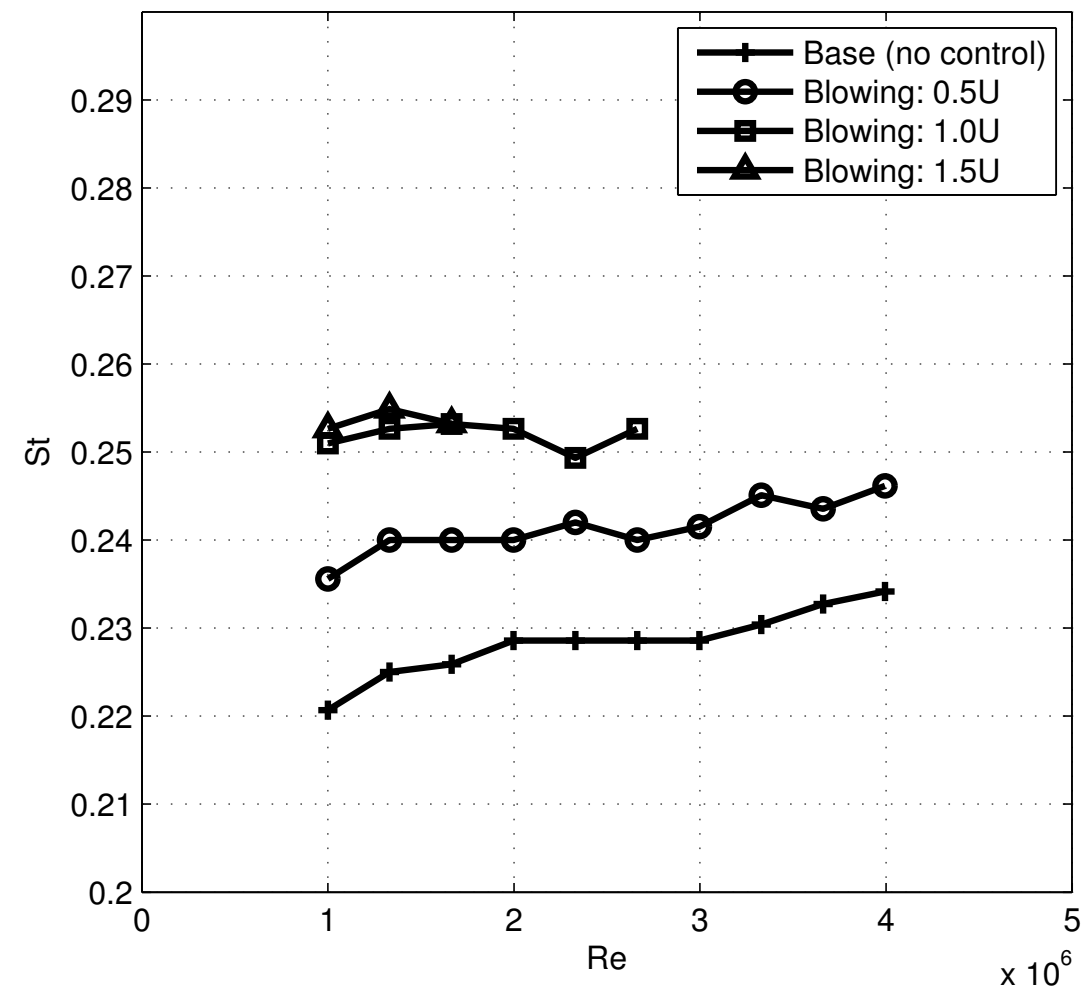

Figure 3.15: Graphical depiction of Strouhal number data from Table 3.2 
causes the downward trend in each data set as Reynolds number increases. For example, the base case has an average drag coefficient of 1.409 at the lowest Reynolds number tested, but this value decreases steadily to 1.172 at the highest Reynolds number tested. Looking back at Figure 3.11 the pressure coefficient is slowly altered by increases in Reynolds number, but by the time the Reynolds number reaches four million the pressure coefficient distribution looks very similar to that of the suction actuated case at the lowest Reynolds number, depicted in Figure 3.12. As is expected from the similarities of the pressure coefficient plots, these two data points have very similar drag coefficient values (1.172 vs. 1.167$)$.

The much bigger observation comes when the large drops in $C_{d}$ are facilitated by each successive increase in suction or blowing velocity, relative to the free-stream. In addition, another phenomenon developed as the suction velocity or Reynolds number were increased. At a certain combination of these two parameters the shedding was stopped, represented in Tables 3.1 and 3.2 as well as Figures 3.13 and 3.15 by a lack of Strouhal number value at that specific data point. At the point in which the actuator caused the wake shedding to cease, a significant decrease in drag was noted that was much more profound than even the previously mentioned drops at each new power level.

Taking both data sets and analyzing them concurrently, a notable pattern arises for the optimum drag reduction techniques. Although admittedly more pronounced in the blowing jet plots, both sets of data have sharp drops in drag coefficient at a certain point in their testing cases. As it turns out, these efficient decreases in drag correspond to a cease in shedding in all capacities. The data indicates that the suction actuator cannot stop the shedding until later Reynolds numbers when compared to 
the jet, however once shedding has ceased, the drag reduction capabilities of both actuators stagnate. Despite this, an increase in actuator power has little effect on final drag coefficient after shedding has ceased.

Additionally, the Strouhal number sees fluctuations as the actuator power and Reynolds number are changed. At a constant level of actuator power and an increase of Reynolds number, the Strouhal number sees a slight overall increasing trend. Alternatively when actuator power is increased the entire set of Strouhal numbers for the range of Reynolds numbers is increased as a whole much more so than the local changes from Reynolds number. Analysis of this trend shows an ever-increasing Strouhal number up until the point where shedding ceases, and therefore Strouhal number no longer exists.

Although a few notable pressure coefficient plots of the rear model have been shown already, a brief comparison of these two actuators effects is seen in Figure 3.16. It is evident that the two actuators have very different methods of backpressure increase on the model and effective drag reduction. However, despite the pressure coefficient profiles looking remarkably different in both shedding and non-shedding modes, the overall effect on drag remains the same.

The data indicates that there is a certain relationship between actuator power and Reynolds number, albeit different for the jet vs suction. Once this hypothetical relationship has been satisfied, the flow field drastically changes from one of alternating shedding vortices to that of the steady state shown in Figures 8 and 10, and also correlates to the sharp decrease in drag coefficient. Since these critical combinations occur at different Reynolds numbers, it is safe to assume that in terms of efficiency, one would need to consider both jet and suction actuators operating in tandem (yet 


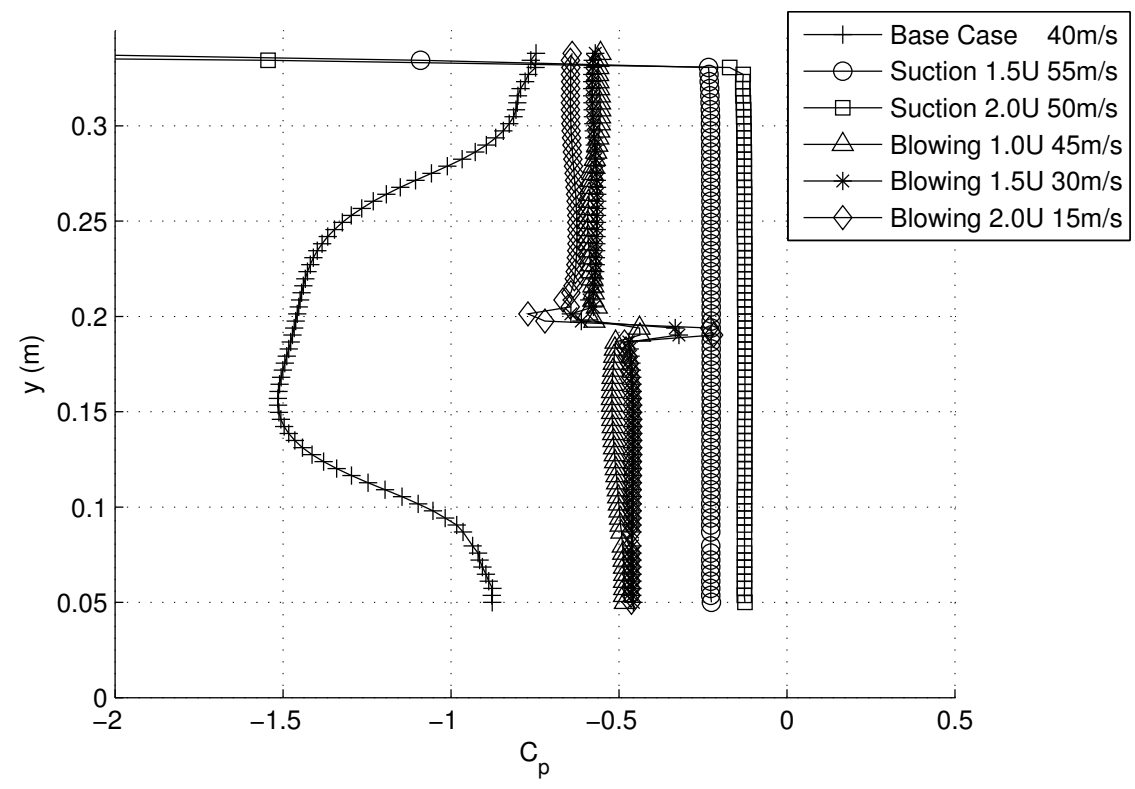

Figure 3.16: Non-shedding pressure coefficient for both actuators

not at the same time). For example, at the lower end of the Reynolds number spectrum (relatively), the suction actuator produces a larger decrease in drag coefficient than the equivalent jet actuator, assuming equal velocities equates to equal power. But as the Reynolds number is increased the jet actuator reaches this critical combination and shedding ceases in these cases, drastically reducing the drag coefficient and making the jet more effective than the suction at an equal power level. Finally looking at the larger end of the Reynolds number spectrum it is clear that once the actuator has ceased the vortex shedding of the flow, the body does not see any more decrease in drag despite the increase in Reynolds number, unlike the case of shedding suction actuated flow. If a moderate power level is required the jet is again a better 
choice, however if more power is available, then the suction will produce a drop in drag coefficient from the prevention of shedding that even exceeds the jet actuator.

Following on these aforementioned conclusions and using the vorticity generation plots from the steady state cases, it is apparent that given enough power and a high enough Reynolds number both actuators will be effective at stopping the vortex shedding off the rear end of the bluff body. In the future this could lead to experiments done using feedback control loops in which the Reynolds number is an input and the control scheme is used to determine the optimum actuator and power to cease shedding to achieve maximum drag reduction. Going one step further could also include a combination of both actuators at once. Although both seek to increase the back pressure by ultimately stopping vortex shedding, the way they approach this goal is different, as shown in Figure 11. Therefore, the hypothesis on whether the combination would be more or less effective rests on whether the two actuators would interfere with each other constructively or conversely. Effectively, the merit of such an experiment would be heavily based on if the combined power efficiency was determined to be at an advantage. The hypothesis that can be made here is that this effect of stopping vortex formation to reduce drag can be extended to other bluff bodies and even to other control methods. Any such testing on these characteristics would only further validate the underlying theory.

\subsection{Multiple Simultaneous Actuators}

Up until this point the relative efficiencies of the control schemes have not been an integral part of this investigation. The different cases are simply evaluated against each other via keeping the input velocity constant, thereby equating to the same 
power requirements. Following on the heels of Chapter 3.3.3, a brief investigation into utilizing both the suction and blowing actuators simultaneously is now presented. The ultimate goal of this section will be to couple these control schemes in order to utilize the strengths of both at either end of the Reynolds number spectrum. The energy used for the control (with isentropic jet velocity $u_{j e t}$ ) is:

$$
P_{\text {jet }}=\frac{1}{2} \dot{m}_{j e t} v_{\text {jet }}^{2}
$$

When considering only two jets to be active at one time (Suction jet at the top corner and blowing jet at the center rear) the equation for equivalent power becomes:

$$
P_{\text {total }}=P_{\text {jet }}+P_{\text {suction }}=\frac{1}{2} \dot{m}_{\text {jet }} v_{\text {jet }}^{2}+\frac{1}{2} \dot{m}_{\text {suction }} v_{\text {suction }}^{2}=\frac{1}{2} \dot{m}_{\text {total }} v_{\text {total }}^{2}
$$

which, when equal mass is assumed, relates the relative velocities in the following way:

$$
v_{\text {jet }}^{3}+v_{\text {suction }}^{3}=v_{\text {total }}^{3}
$$

Table 3.3 shows this new data in tabular form for the four different velocity specifications relative to the freestream velocity. This same data is also shown in Figures $3.17,3.18,3.19$, and 3.20 which all represent equivalent required power for each set of control schemes.

From Table 3.3 it is clear that the same mechanism influencing the sudden drop in drag coefficient in section 3.3.3 (stoppage of shedding) is still the main contributor when both controls are active at once. For the case of controls powered by $0.5 \cdot U_{\infty}$ the drag coefficient still sees a decrease in overall magnitude however the shedding stoppage phenomenon does not develop, as was also the case for each actuator alone. However, since the Reynolds number effect is the cause for the downward trend inside 


\begin{tabular}{ccccccccc} 
& \multicolumn{2}{c}{ Both at $0.397 \cdot U_{\infty}$} & \multicolumn{2}{c}{ Both at $0.794 \cdot U_{\infty}$} & \multicolumn{2}{c}{ Both at $1.19 \cdot U_{\infty}$} & \multicolumn{2}{c}{ Both at $1.587 \cdot U_{\infty}$} \\
\hline Reynolds Number & $C_{d}$ & St & $C_{d}$ & St & $C_{d}$ & St & $C_{d}$ & St \\
\hline $1.00 \mathrm{E}+06$ & 1.216 & 0.243 & 1.020 & 0.267 & 0.427 & 0.410 & \\
$1.33 \mathrm{E}+06$ & 1.204 & 0.244 & 0.978 & 0.267 & 0.334 & 0.330 & \\
$1.66 \mathrm{E}+06$ & 1.176 & 0.245 & 0.926 & 0.268 & 0.297 & 0.308 & \\
$2.00 \mathrm{E}+06$ & 1.142 & 0.246 & 0.876 & 0.267 & 0.292 & 0.245 & \\
$2.33 \mathrm{E}+06$ & 1.098 & 0.246 & 0.828 & 0.265 & 0.289 & 0.240 & \\
$2.66 \mathrm{E}+06$ & 1.053 & 0.248 & 0.345 & & 0.287 & 0.240 & \\
$3.00 \mathrm{E}+06$ & 1.006 & 0.246 & 0.343 & & 0.285 & 0.239 \\
$3.33 \mathrm{E}+06$ & 0.957 & 0.250 & 0.341 & & 0.285 & 0.240 & \\
$3.66 \mathrm{E}+06$ & 0.907 & 0.249 & 0.341 & & 0.285 & 0.240 & \\
$4.00 \mathrm{E}+06$ & 0.845 & 0.253 & 0.340 & & 0.285 & 0.242 & \\
\hline
\end{tabular}

Table 3.3: Data from simultaneous control schemes (absence of St entry indicates shedding stoppage)

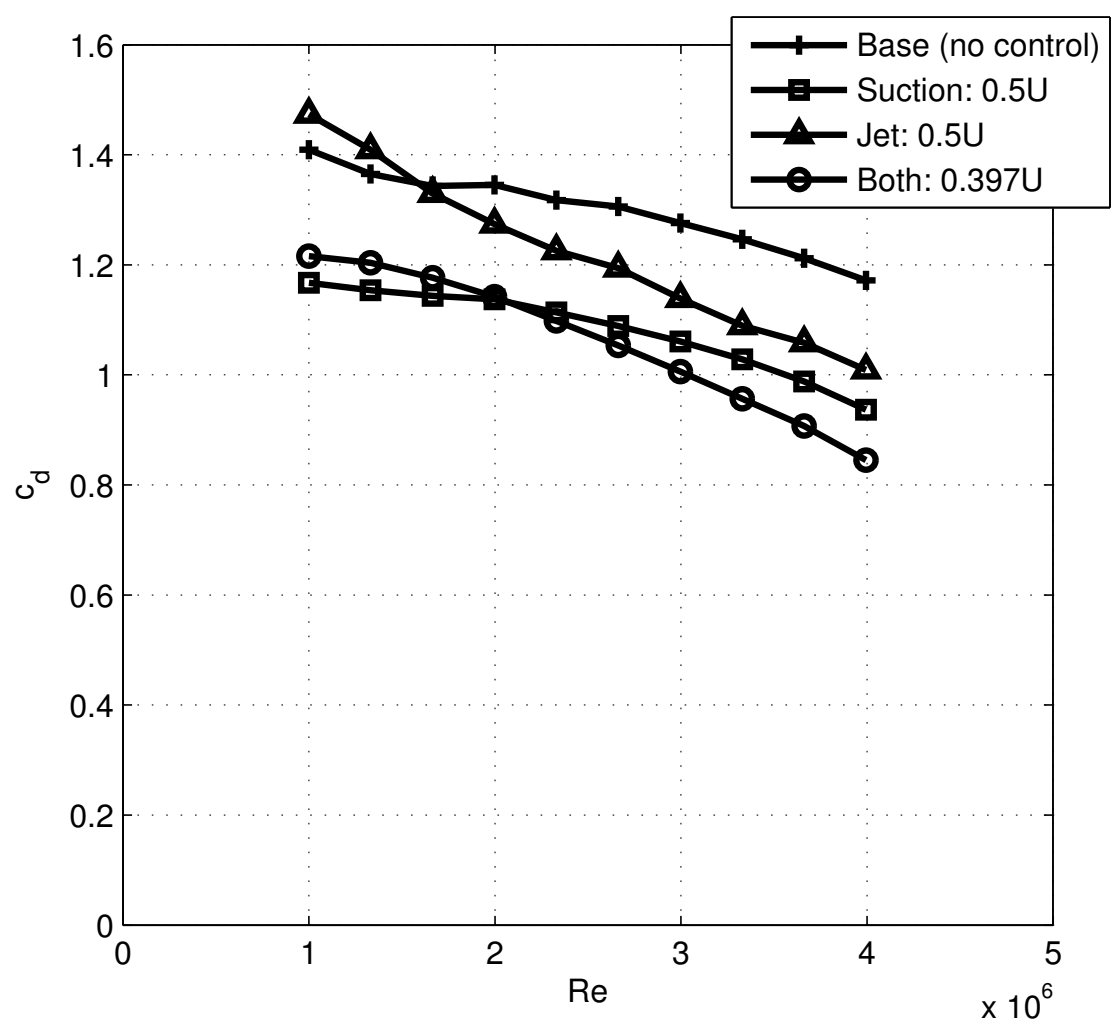

Figure 3.17: Drag coefficient data for equivalent power of $0.5 \cdot U_{\infty}$ 


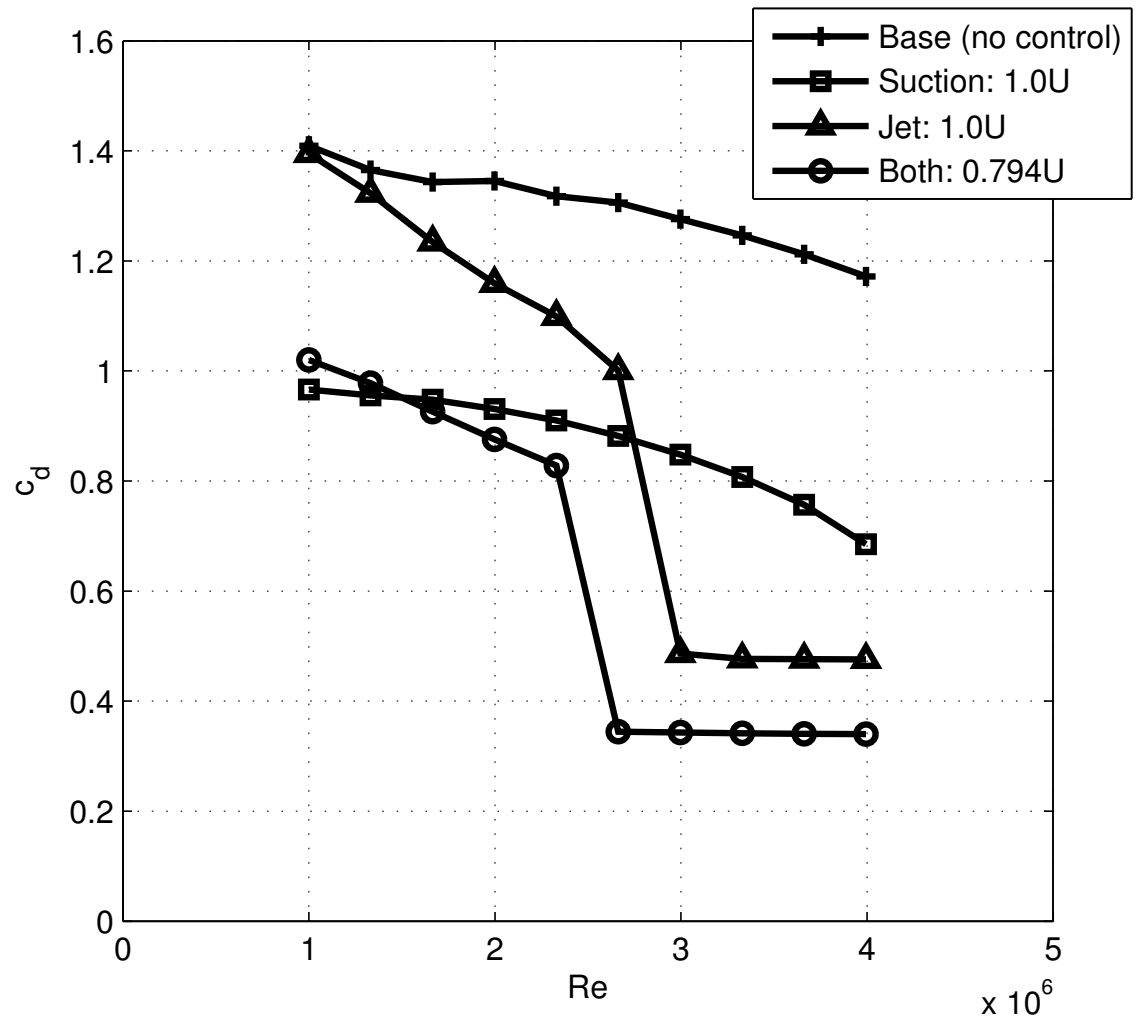

Figure 3.18: Drag coefficient data for equivalent power of $1.0 \cdot U_{\infty}$ 


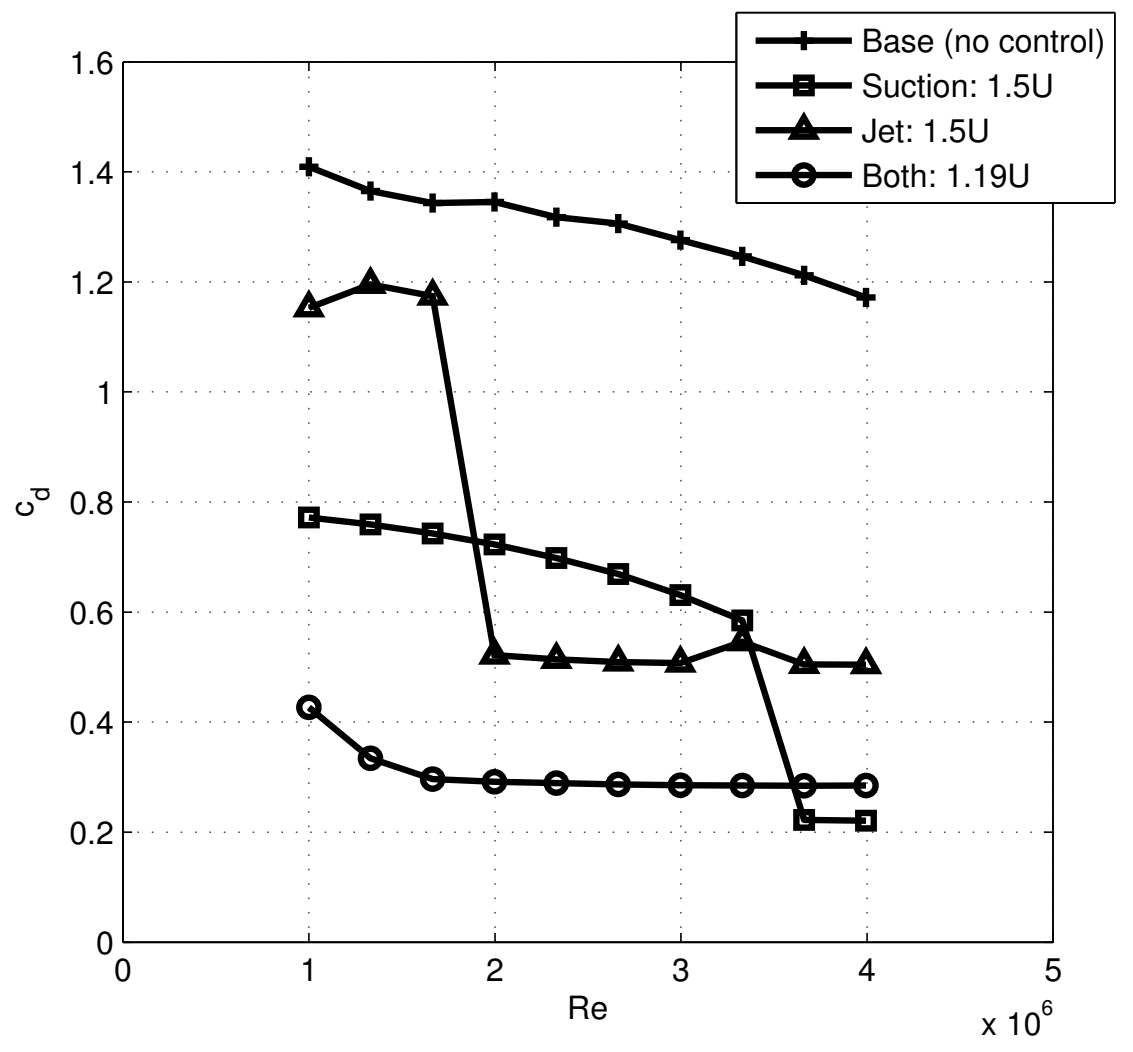

Figure 3.19: Drag coefficient data for equivalent power of $1.5 \cdot U_{\infty}$ 


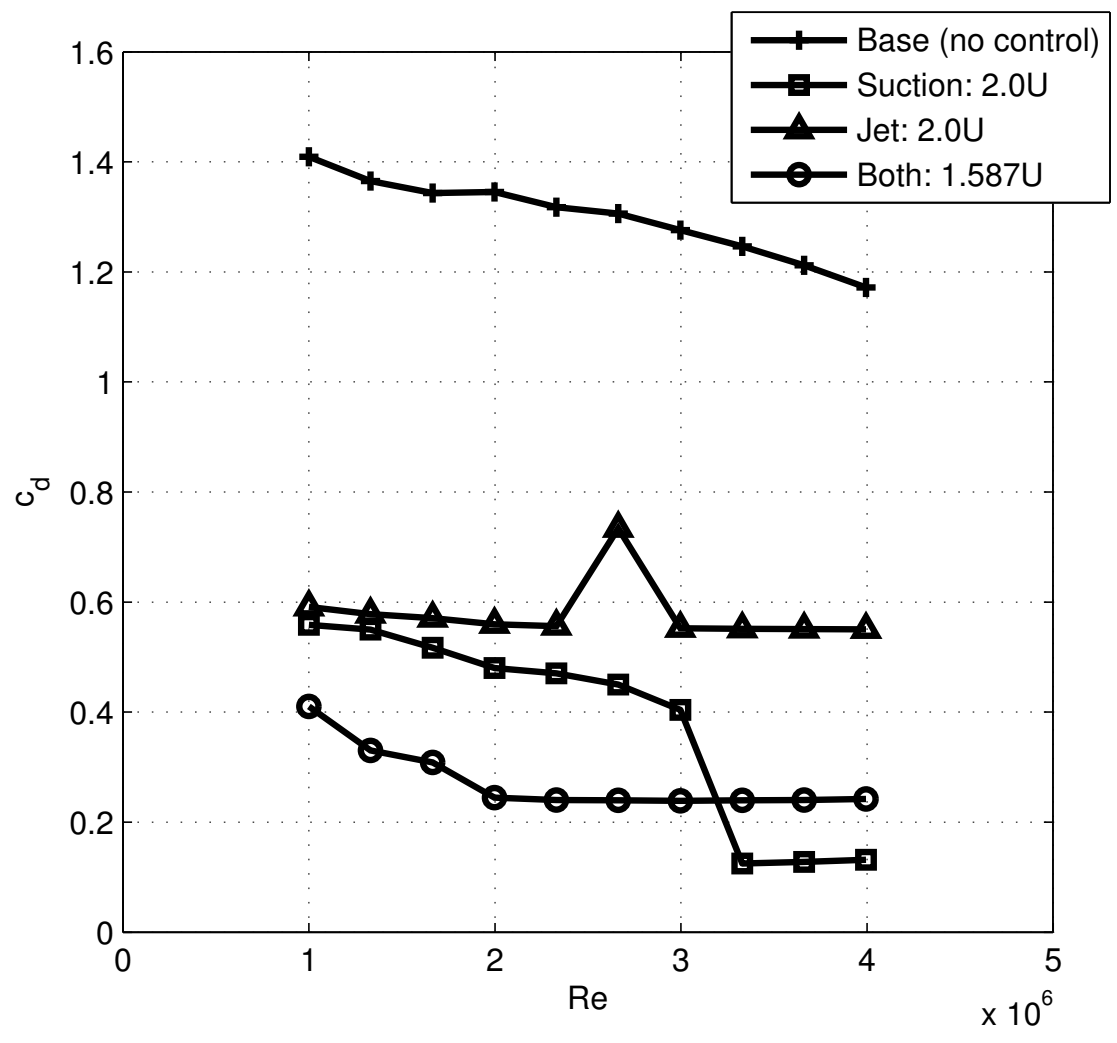

Figure 3.20: Drag coefficient data for equivalent power of $2.0 \cdot U_{\infty}$ 
each data set, and the effect mimics the effects caused by the suction actuators, it can be assumed that at a certain higher Reynolds number even these data sets would experience a stoppage of shedding, which has been shown experimentally by Roshko [2]. At relative powers of 1.0 to 2.0 the effects are magnified by employing simultaneous actuation. In the other three remaining cases the expected drop in drag coefficient from shedding stoppage occurs at lower Reynolds numbers when the controls are combined than with either of them separately. This correlates directly to a lower drag coefficient over a wider range of Reynolds numbers by utilizing both controls at once versus each on their own, keeping in mind all scenarios are evaluated with equivalent power in mind.

Recalling from section 3.3.3 it was stated that at the lower end of the Reynolds number spectrum (relatively), the suction actuator produced a larger decrease in drag coefficient than the equivalent jet actuator. But as the Reynolds number was increased the jet actuator reached this critical combination before the suction and shedding ceased in these cases, drastically reducing the drag coefficient and making the jet more effective than the suction at those higher velocities. However, at the extreme Reynolds numbers when both actuators are effective in stopping the shedding, the suction control will reduce the drag coefficient more than the jet. By coupling these two actuators the strengths of each were indeed combined as was hoped at the start. The ability of the jet actuator to force a non-shedding state combined with the suction controls ability to reduce the drag coefficient even lower once in this state has allowed for a blanket improvement of the singular control scheme in almost all cases tested. 


\section{Chapter 4: Three-Dimensional Simulations}

\subsection{Simulation Setup}

Following Chapter 3 a new set of investigations has been performed involving the addition of another dimension to the Ahmed model, making the model now truly an accurate representation of the real world models tested in experiments by Ahmed and others. Throughout this chapter ANSYS FLUENT remains as the computational solver, however ANSYS ICEM CFD is used to generate the three-dimensional computational domain instead of the built-in mesher of ANSYS Workbench. Two sets of experiments are performed, one at a Reynolds number of 500,000 and another at 4 million. This distinction was chosen a priori to coincide with available research for validation and comparison, and also to create flows relevant to the typical highway driving environment. At each Reynolds number a RANS simulation of the base case is run as well as another using LES. Additionally, another LES simulation is performed at the higher Reynolds number with a suction control scheme implemented.

\subsubsection{Discretization and Boundary Conditions}

Many of the geometric aspects of this new model are simply an extension of the two-dimensional model discussed previously. For example, the length and height of 
the model are still $1.044 \mathrm{~m}$ and $0.288 \mathrm{~m}$ respectively, however the added dimension width is $0.389 \mathrm{~m}$. A schematic of the original Ahmed model that this simulation is based on is shown in Figure 4.1. Without the struts, the model sits at a distance above the ground of $0.05 \mathrm{~m}$ with edges rounded at the front with a radius of $0.1 \mathrm{~m}$ and the back squared off to a zero degree slant.

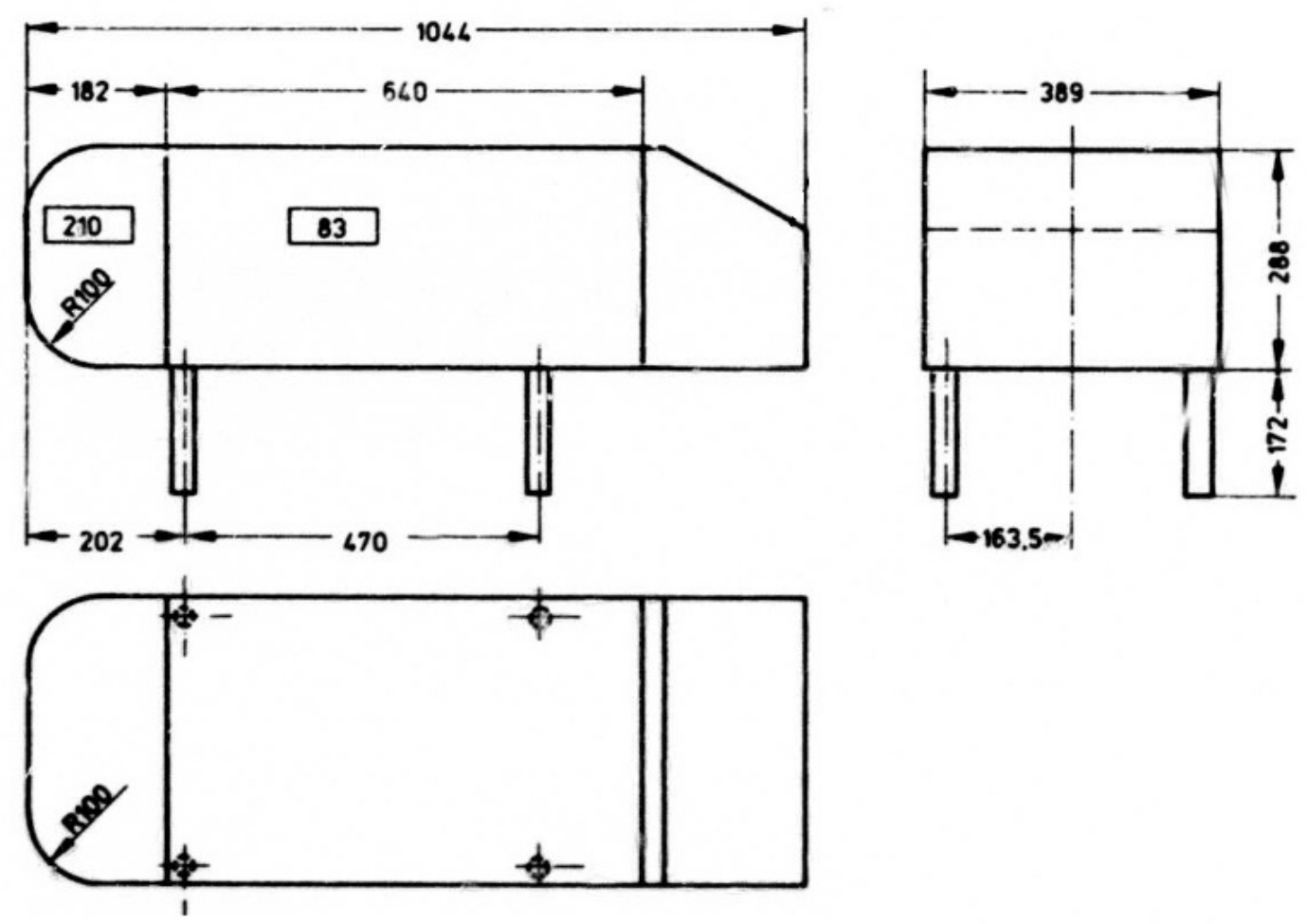

Figure 4.1: Original Ahmed model geometry

The computational domain is meant to represent a typical highway scenario and is based on a similar setup by Eichinger [21], shown as a schematic in Figure 4.2 which 
also depicts the numerical boundary conditions. The side walls sit at a distance of $2.8 \mathrm{H}$ from the sides of the model, the upper boundary is located $3.69 \mathrm{H}$ from the top surface of the model, the upstream distance is $6.38 \mathrm{H}$ and it is $17.26 \mathrm{H}$ downstream from the rear. The model itself as well as underneath the model are treated as no-slip wall conditions, with the lower wall given a velocity equal to that of the inlet in the stream-wise direction. At the upstream boundary a constant inlet velocity condition is prescribed. Downstream the pressure outlet with zero gage pressure is used. With the amount of distance between the model and these boundaries, it is safe to assume no disturbances from the inlet or outlet will effect the domain. On the remaining two side walls and upper surface the symmetry condition is used. A more typical 3/4 open wind tunnel model is usually used in such cases by prescribing the outer boundaries as far-field pressure conditions as they are more akin to real world flow physics, however because of the requirements of such a condition the mesh cell count would increase drastically and the turn-around-time would be too long for the limited resources available.

\subsubsection{Transient Large Eddy Simulations}

A multi-view schematic of the LES mesh is presented in Figure 4.3. This schematic shows the unstructured tetrahedral domain used for the solver during the LES computations. Several additional refinement zones are employed in order to completely capture the flow field physics in the general vicinity of the Ahmed body in addition

to the wall $y^{+}$value of approximately 1.0 on both the ground plane and the model, the standard for LES computations. The total cell count is approximately 22 million. 


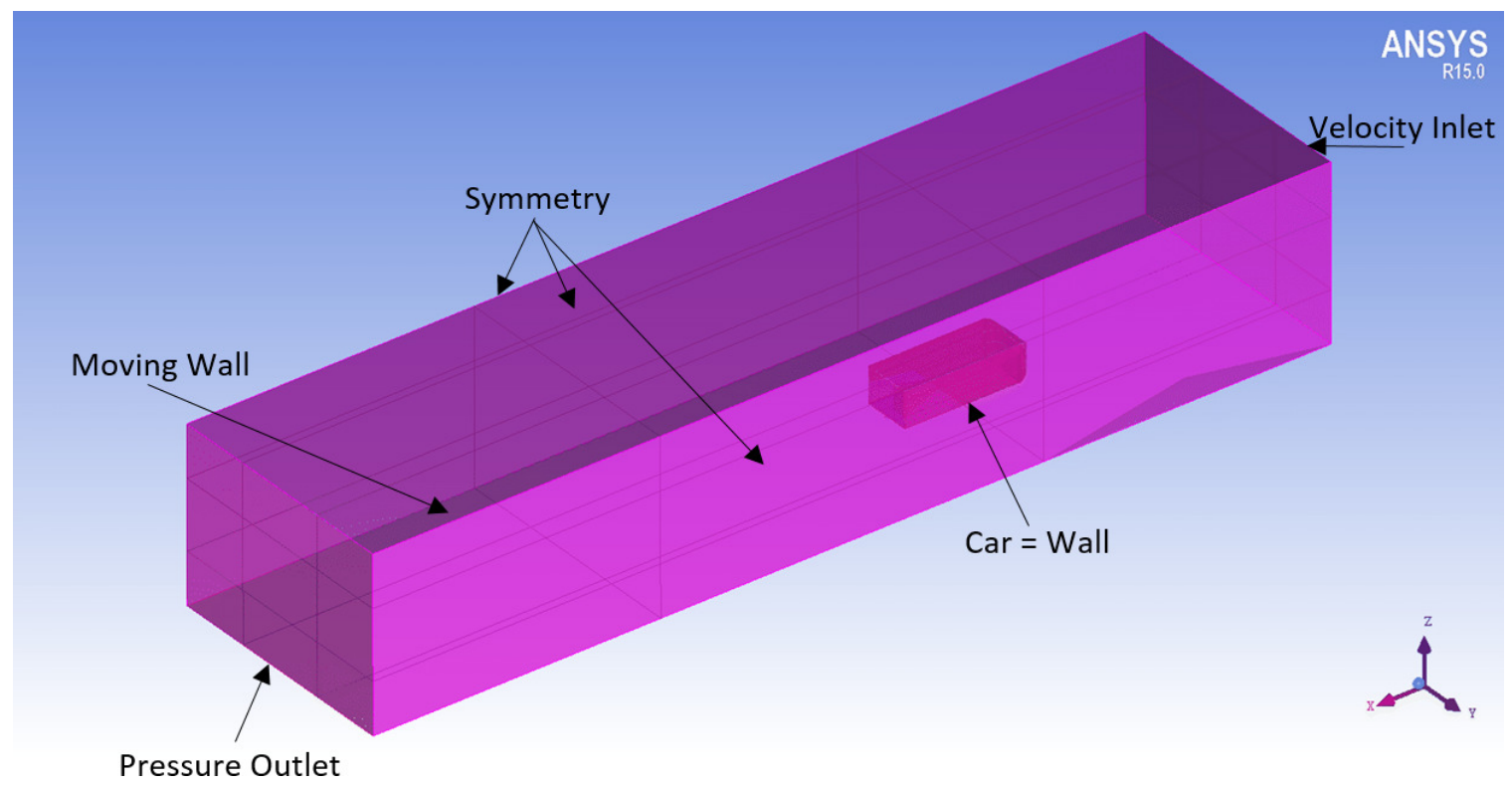

Figure 4.2: Numerical boundary conditions for 3D simulations

\subsubsection{Unsteady RANS Simulations}

Although the RANS mesh is undoubtedly different from the LES mesh, the changes are not especially visible to the naked eye and therefor a second multi-view schematic will not be shown. As was stated, the RANS mesh is modeled closely after the LES mesh with the same refinement zones and general shape. However, due to the different limitations on the $k-\omega$ SST versus a LES model the $y^{+}$value of 1.0 was altered to an average of 30 and the refinement zones relaxed. With these alterations the RANS mesh cell count was only 15 million.

An impulsively started inlet is used to initialize the flow simulation in the case of the $k-\omega$ SST models. A second order implicit scheme is used along with the PISO pressure-velocity coupling and central differencing. A time step of 0.001 seconds carries the solution with residuals on the order of $10^{-6}$ until a steady state has been reasonably achieved. A plot showing drag coefficient vs time is shown in Figure 4.4, 

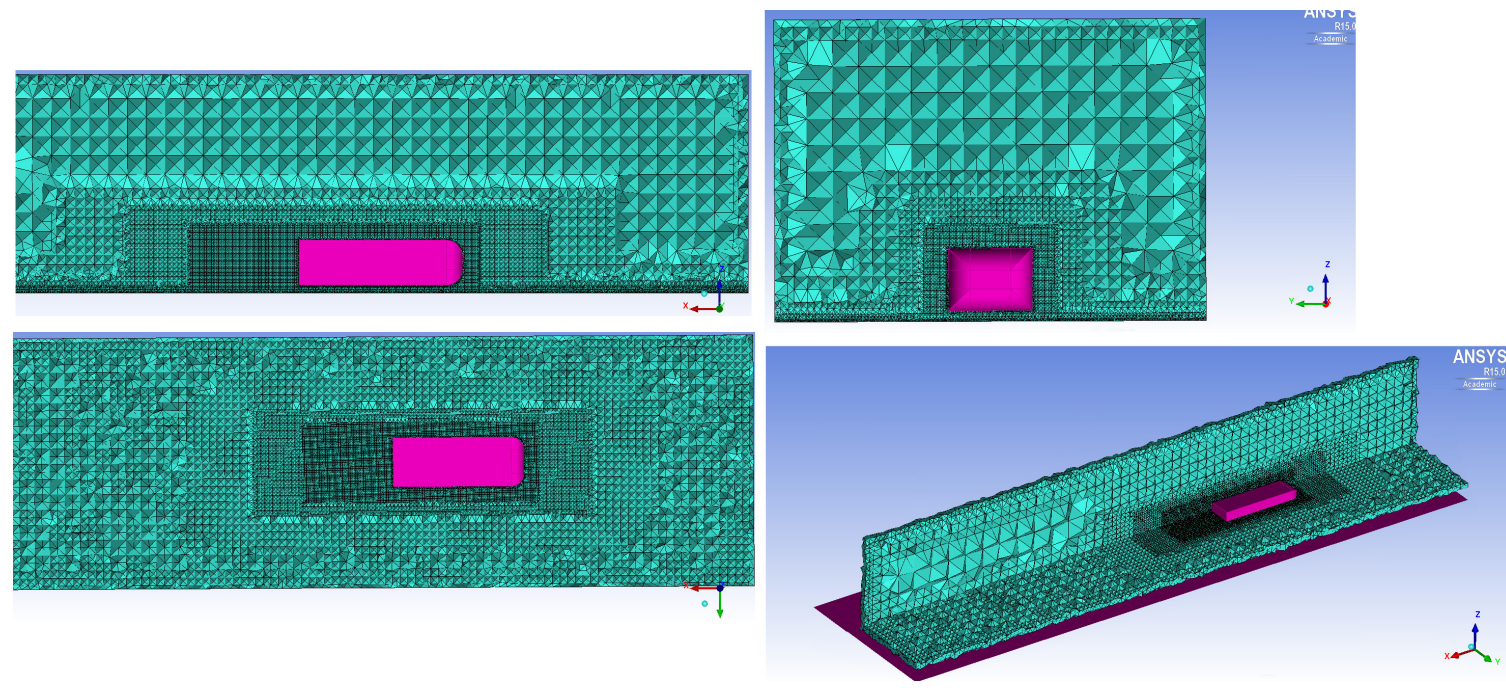

Figure 4.3: Multi-view ICEM mesh for numerical simulation

further supporting the accuracy of the simulation as the drag coefficients coincide with many external experiments performed [11, 14, 16, 20, 35]. Small inconsistencies in overall drag coefficient can be attributed to slightly differing model conditions, including open vs closed test sections and moving vs non-moving ground.

At this point, the solution iterates further to reveal time-averaged data for components of interest such as velocity and pressure. Figure 4.5 shows a comparison of plane-projected streamlines from the lateral and horizontal planes at the near wake of the vehicle, between the simulation run here and that of Eichinger [21]. These projected streamlines give rise to two vortex foci in the near wake flow. The upper vortex tends to extend further into the mean flow area, with a core located at approximately $\mathrm{z} / \mathrm{H}=0.97$ and $\mathrm{x} / \mathrm{H}=0.82$, while the lower seems to be much more flattened with an approximate location of $\mathrm{z} / \mathrm{H}=0.20$ and $\mathrm{x} / \mathrm{H}=0.97$. These two sets of vortices combine together with those shown in the lower half of the figure (located at approximately $x / H=0.66$ and $y / H= \pm 0.46)$ to form a single ring-shaped vortex. A visualization 


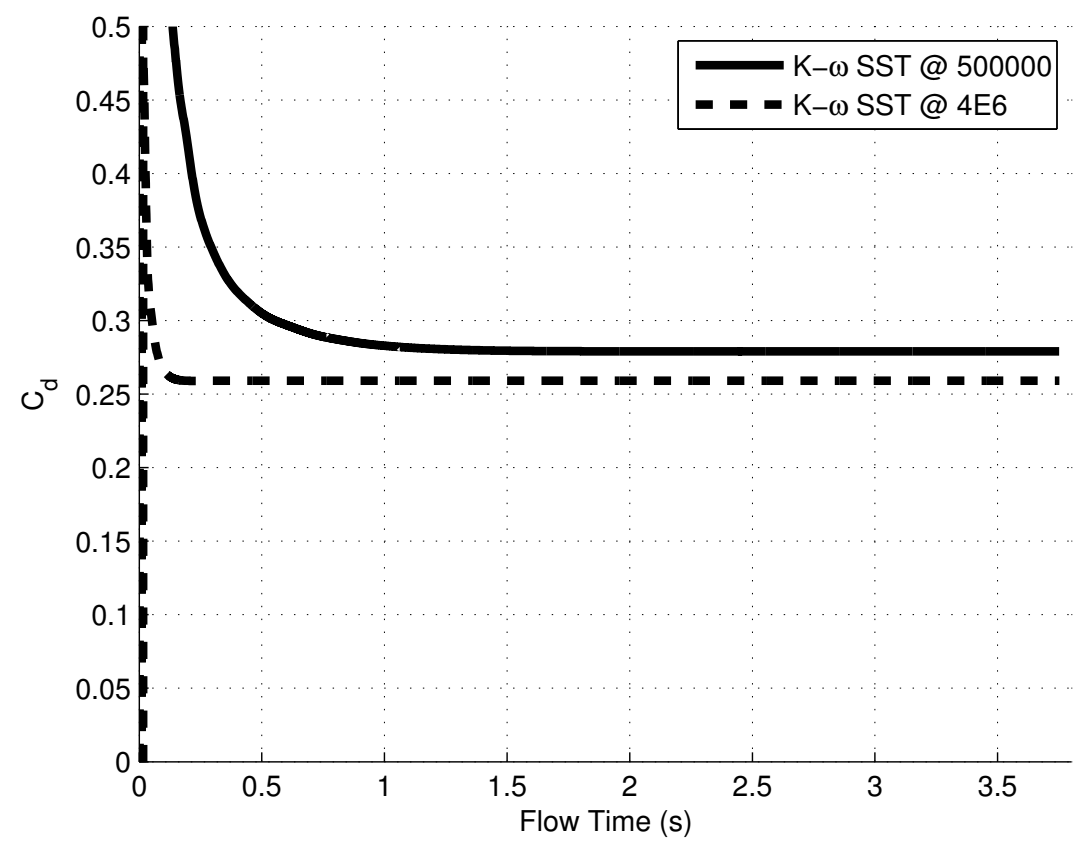

Figure 4.4: Transient drag coefficient for RANS simulations

of this low pressure vortex is shown in Figure 4.6 represented by an isosurface of negative pressure colored by velocity magnitude. A free stream stagnation point can be observed at $\mathrm{z} / \mathrm{H}=0.20$ and $\mathrm{x} / \mathrm{H}=1.61$, along with another stagnation point on the rear surface at $\mathrm{z} / \mathrm{H}=0.45$. When compared, the location of these specific identifying critical points coincide closely with those of reference [21].

In Figure 4.7 the surface-projected streamlines based on average values are shown on the rear surface, in addition to the average pressure distribution (warmer colors indicate the areas of higher pressure). The streamlines all emanate from the stagnation point colored in red near the center of the rear wall. This stagnation point is located in the exact location predicted by the two lateral and horizontal plane streamline plots in Figure 4.5 and center of the low pressure ring in Figure 4.6. Discrepancies in 

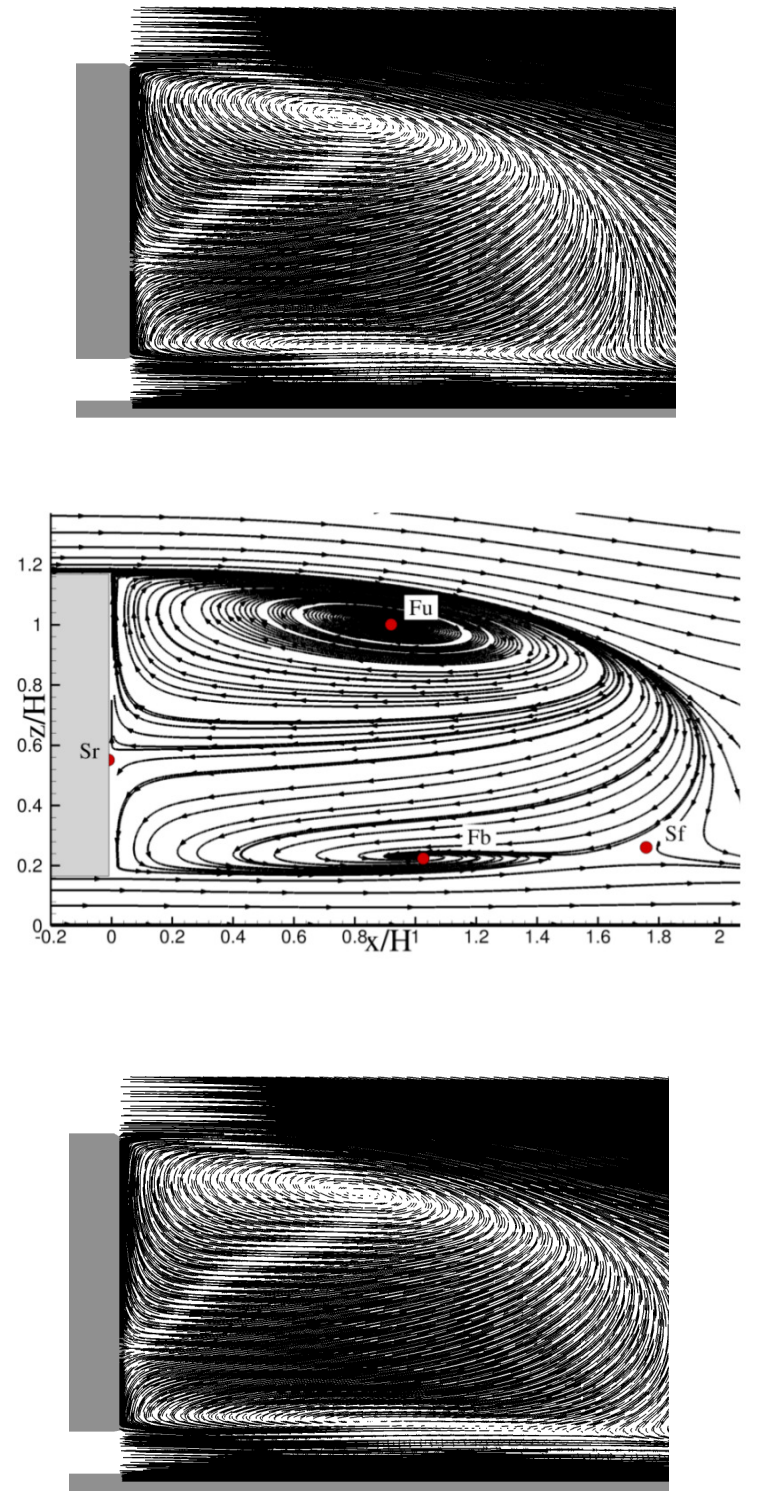
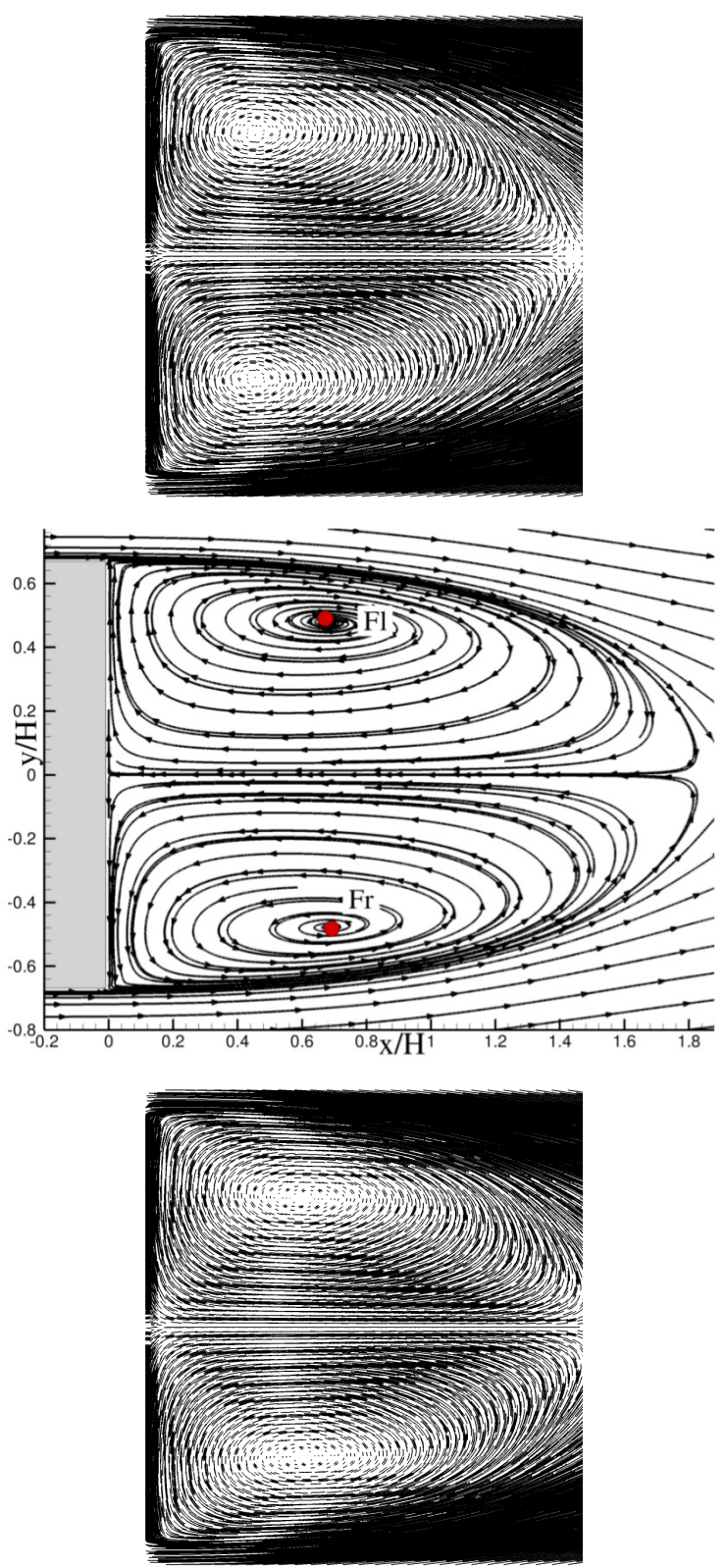

Figure 4.5: RANS, Time-averaged, plane-projected, streamlines: ( $1^{\text {st }}$ Row) Re = 0.5E6, (2 ${ }^{\text {nd }}$ Row) Re $=2.78 \mathrm{E} 6[21],\left(3^{\text {rd }}\right.$ Row $)$ Re $=4 \mathrm{E} 6$

the stagnation point location uncovered by Eichinger can be attributed to differences in moving-ground floor and Reynolds number. 


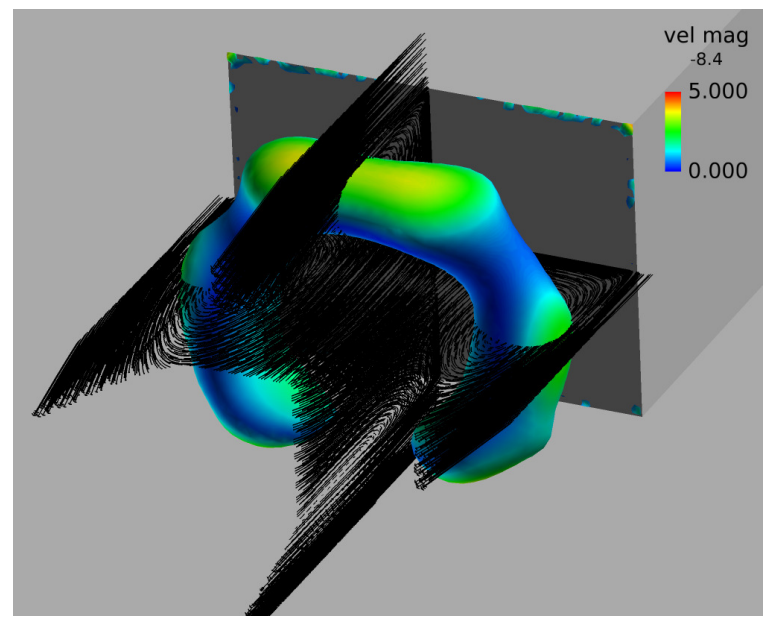

Figure 4.6: Isosurface of pressure in rear wake colored by velocity magnitude

Surface projected streamlines are shown in Figure 4.8 from stream-wise perpendicular planes. From the bottom edges longitudinal vortices are rolling up and convecting downstream. Near to this bigger vortex under the bottom side is a smaller set of secondary structures. Far downstream the whole system forms a pair of counter rotating vortices. Additional reference materials can be seen on the right [21] to support the conclusions made.

The data presented above provides sufficient support that RANS can be used to determine the relative characteristics of the time-averaged flow field. This type of analysis is sufficient for some basic aerodynamic concerns, such as average pressure on the vehicle rear or average drag coefficient to determine efficiency. However, supplementary research involves analyzing the transient data as well, for use in Strouhal number analysis or any kind of pulsed active control scheme. To obtain these kinds of transient results, an LES model must be used. 

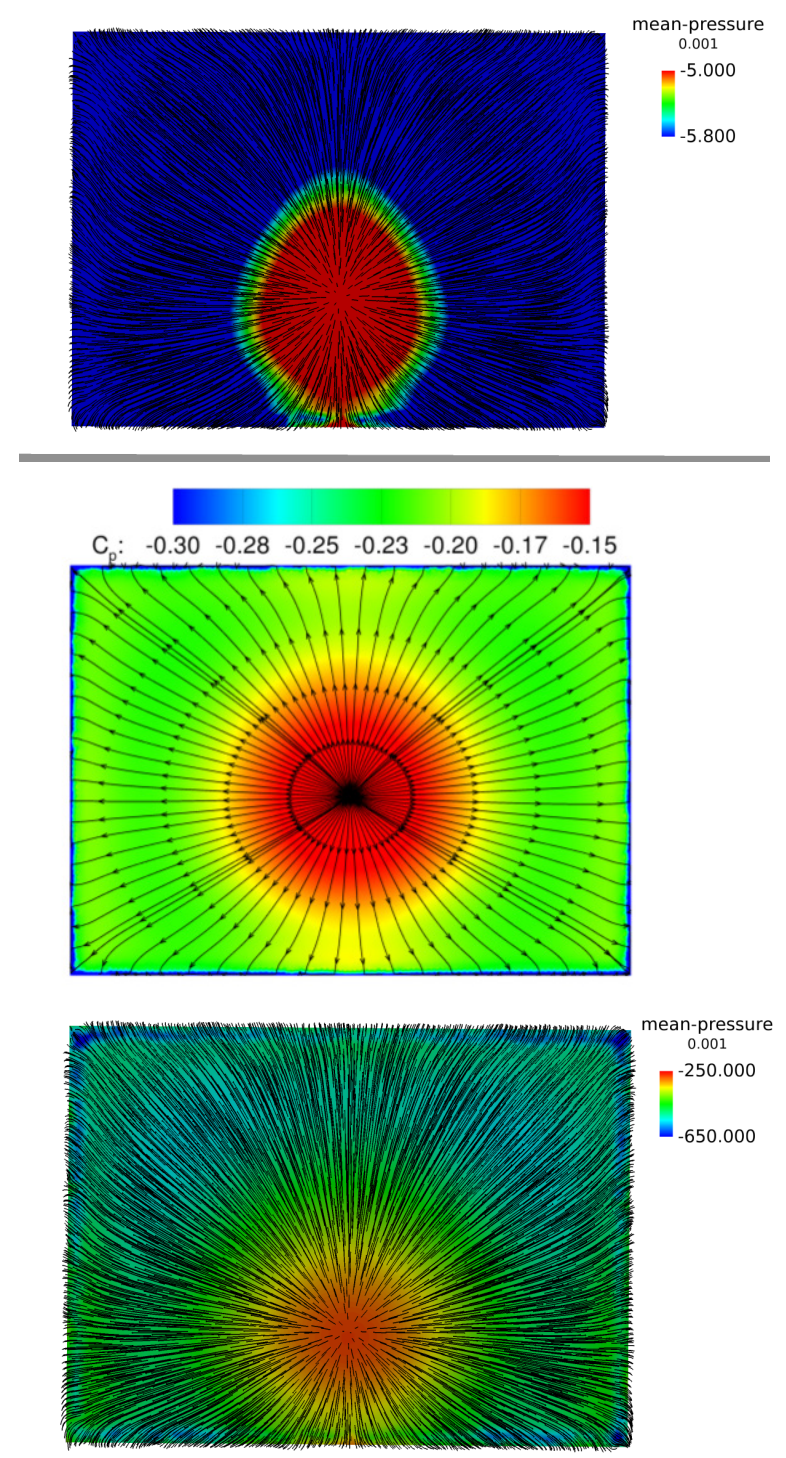

Figure 4.7: RANS, Pressure distribution and streamlines projected to the vehicle rear-end surface: (Top) $\mathrm{Re}=0.5 \mathrm{E} 6$, (Middle) $\mathrm{Re}=2.78 \mathrm{E} 6$ (stationary floor) [21], (Bottom) Re $=4 \mathrm{E} 6$

\subsection{Base Case (no control)}

Due to the different nature of the LES model as opposed to a RANS calculation, some specific numerical details have to be altered to produce an accurate result. For example, a smaller time-step of $0.0001 \mathrm{~s}$ has to be used in the simulations. Coupled 

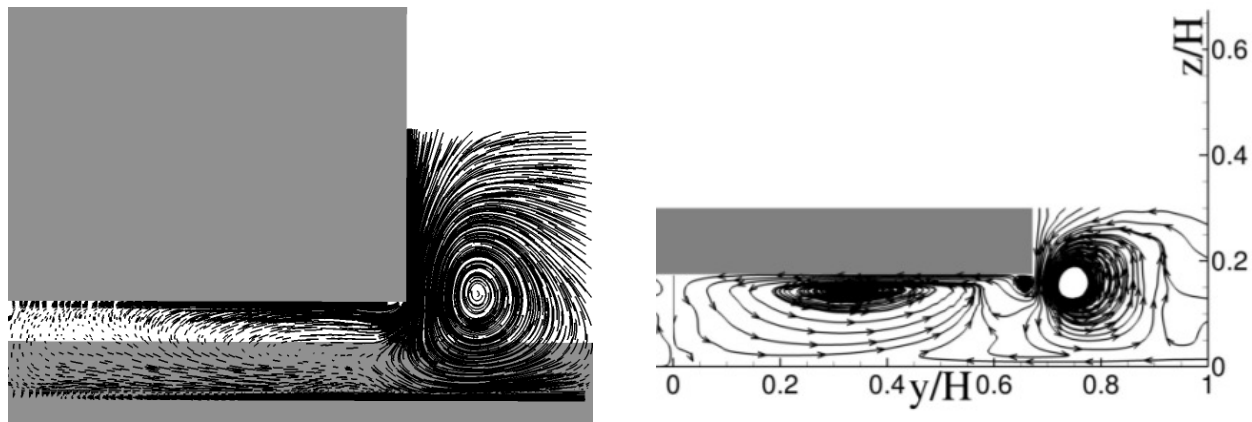

(a) $\mathrm{x} / \mathrm{H}=-0.2$
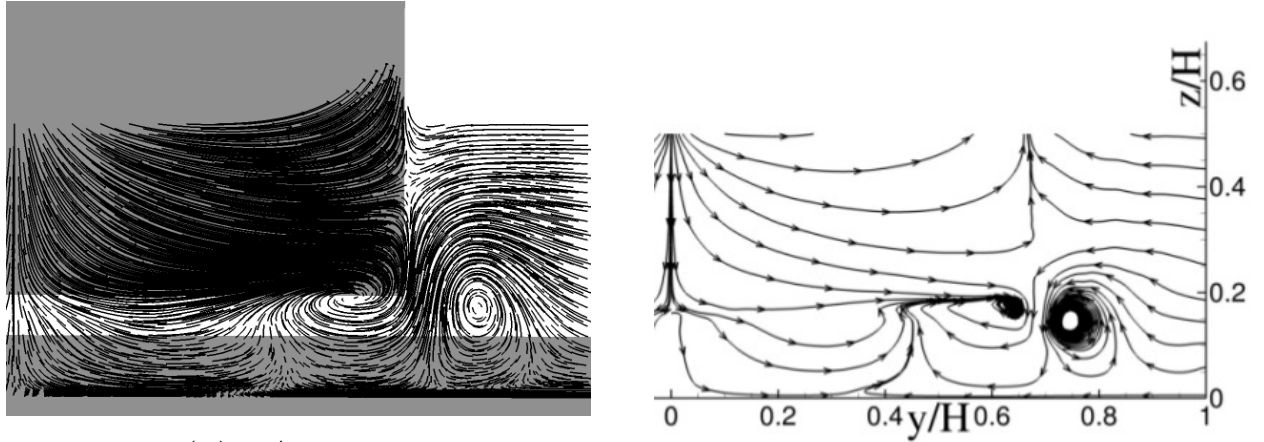

(b) $\mathrm{x} / \mathrm{H}=0.2$

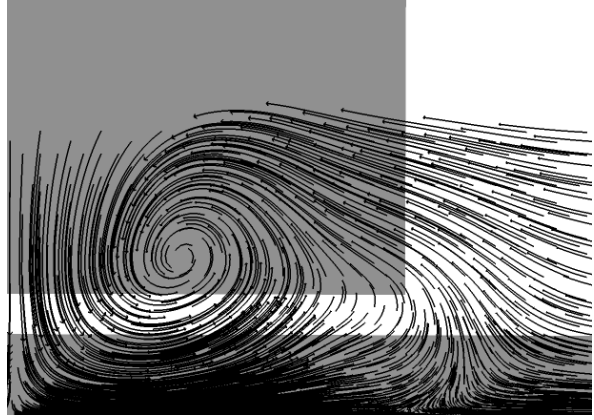

(c) $\mathrm{x} / \mathrm{H}=3$

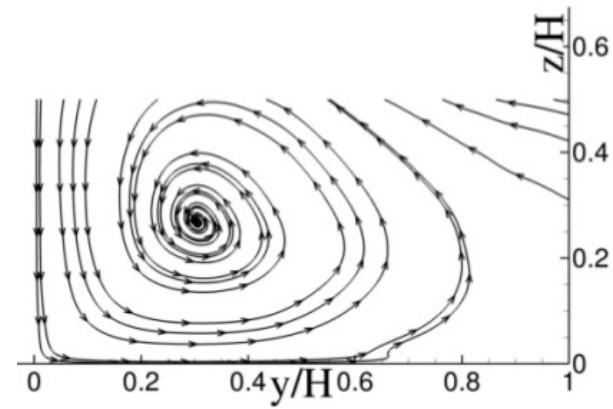

Figure 4.8: Surface projected streamlines from different $\mathrm{x} / \mathrm{H}$ locations, left from RANS simulation at $\mathrm{Re}=0.5 \mathrm{E} 6$, right from $\mathrm{LES}$ at $\mathrm{Re}=0.5 \mathrm{E} 6$ [21]

with the same second order implicit scheme along with the PISO pressure-velocity coupling and central differencing, the LES model is able to reach residuals on the order of $10^{-6}$ similar to the previous work. However, due to the larger cell count and smaller time step it is not practical to wait for the solution to develop into the quasi-steady 
state as it was in the previous case. Instead, the time-averaged results from the $k-\omega$ SST model are used for the initial conditions instead of the impulsively started inlet. Figure 4.9 shows plane-projected time-averaged streamlines similar to those in Figure 4.5 compared with Eichinger [21]. It is critical to see that the dominant ring-shaped vortex structure still remains, however the critical points that create it are in slightly different positions. The upper vortex foci is now at $\mathrm{z} / \mathrm{H}=1.01$ and $\mathrm{x} / \mathrm{H}=0.82$ and the lower at $\mathrm{z} / \mathrm{H}=0.22$ and $\mathrm{x} / \mathrm{H}=1.10$, indicating that the low pressure ring vortex has shifted in the stream-wise direction. Similarly, the vertical-plane free stream stagnation point has moved down the rear side by approximately $10 \%$. These results coincide with findings from Eichinger and serve to validate the model for further use at the higher Reynolds numbers of interest to this Thesis. Due to instability effects the required interval for time averaging is relatively long [21]. In particular attention to the top-down views, insufficient time averaging has given the impression of uneven vortices, due to the transient shedding of the vortices still developing.

In Figure 4.10 the surface-projected streamlines based on average values are shown on the rear surface, in addition to the average pressure distribution (warmer colors indicate the areas of higher pressure). The streamlines all emanate from the stagnation point colored in red near the bottom center of the rear wall. Although the color scale is different between data presented here and that of Eichinger, the relative size and shape of distinguishing high or low pressure fields are very similar.

Surface projected streamlines are shown in Figure 4.11 from stream-wise perpendicular planes. Similar to the RANS simulations, from the bottom edges longitudinal vortices are rolling up and convecting downstream, however LES has managed to also capture additional circulation on the underside of the model. Near to this bigger 

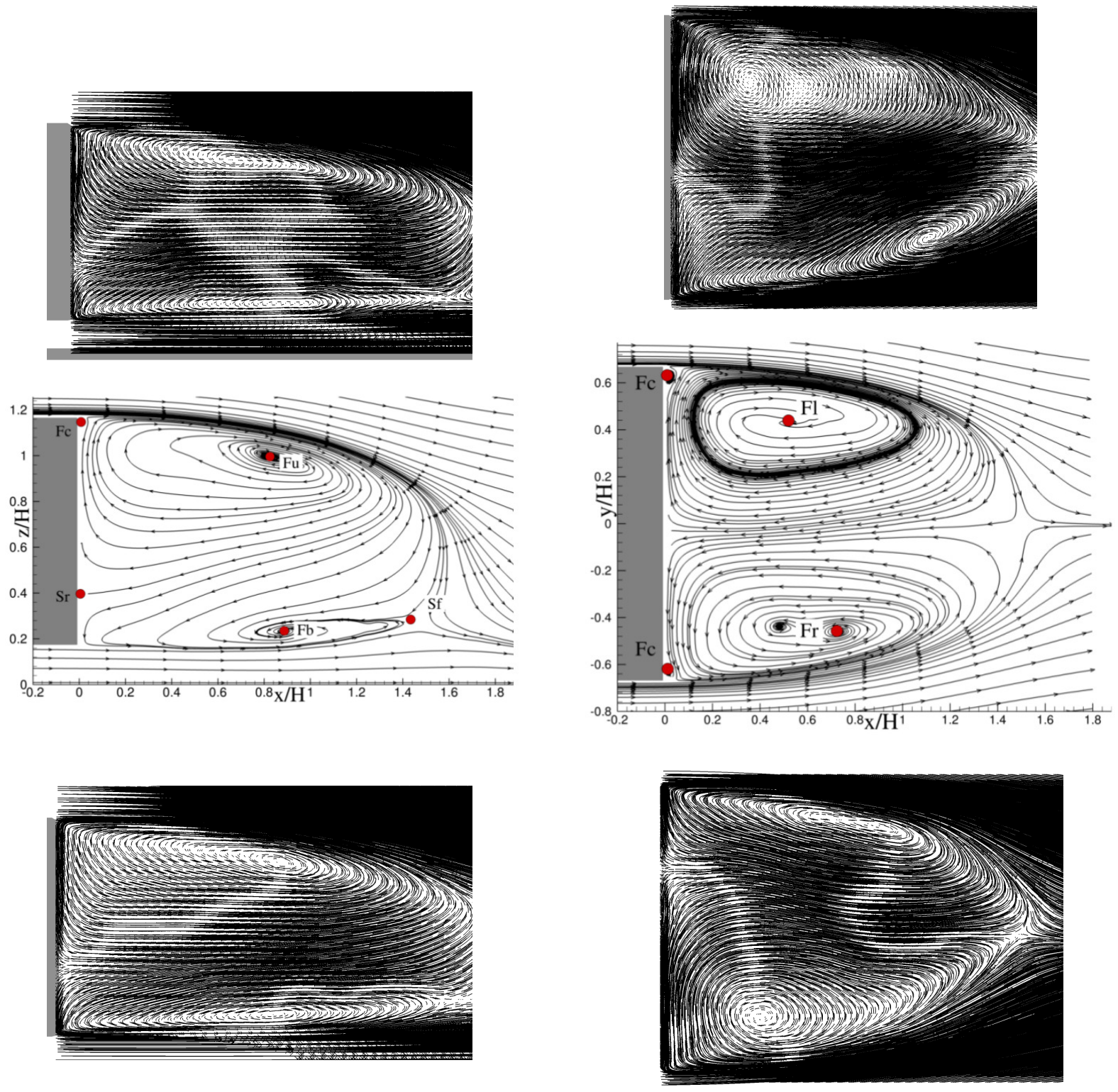

Figure 4.9: LES, Time-averaged, plane-projected, streamlines: ( $1^{\text {st }}$ Row $)$ Re $=0.5 \mathrm{E} 6$, $\left(2^{\text {nd }}\right.$ Row $) \mathrm{Re}=0.5 \mathrm{E} 6[21],\left(3^{\text {rd }}\right.$ Row $) \mathrm{Re}=4 \mathrm{E} 6$

vortex under the bottom side is a smaller set of secondary structures. Far downstream the whole system forms a pair of counter rotating vortices. Again, additional reference materials can be seen on the right [21] to support the conclusions made. 


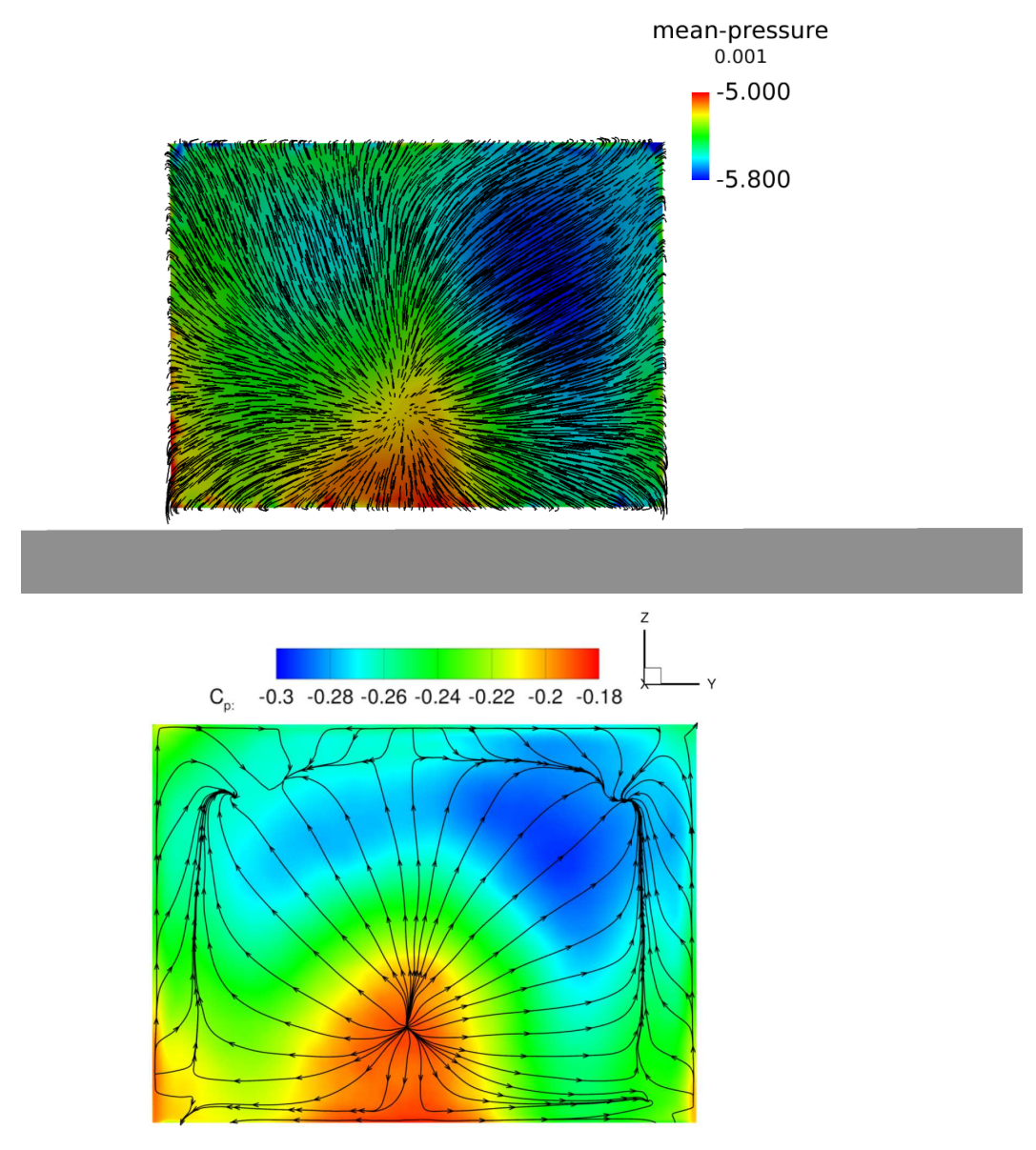

Figure 4.10: Pressure distribution and streamlines projected to the vehicle rear-end surface: (Top) Re $=0.5 \mathrm{E} 6,($ Bottom) $\mathrm{Re}=0.5 \mathrm{E} 6[21]$

\subsubsection{Time-dependent flow features}

Large Eddy analysis also allows the collection of time-dependent data for use in transient shedding and drag analysis or instantaneous variables for a better understanding of the flow field characteristics. Total pressure probes were placed into the wake of the LES base case, located at $\mathrm{x} / \mathrm{H}=0.01 \mathrm{y} / \mathrm{H}=-0.67 \mathrm{z} / \mathrm{H}=0.05$ and $\mathrm{x} / \mathrm{H}=0.85$ $\mathrm{y} / \mathrm{H}=0 \mathrm{z} / \mathrm{H}=0.225$. In Figure 4.12 the unsteady total pressure data from both monitor points is presented. Using the MATLAB Fast Fourier Transform (FFT) function, 


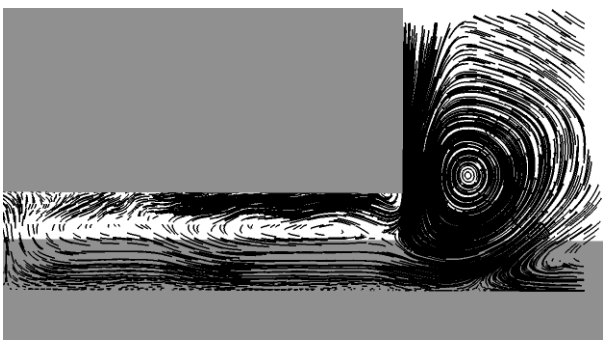

(a) $\mathrm{x} / \mathrm{H}=-0.2$

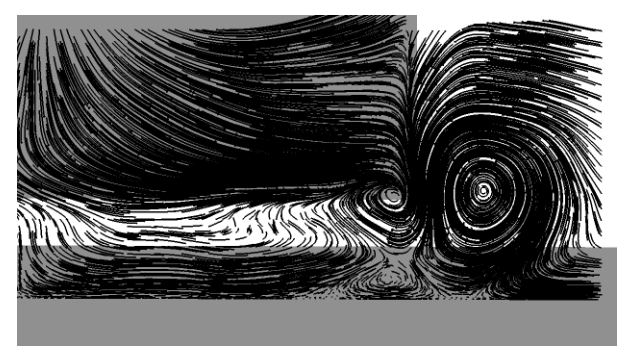

(b) $\mathrm{x} / \mathrm{H}=0.2$

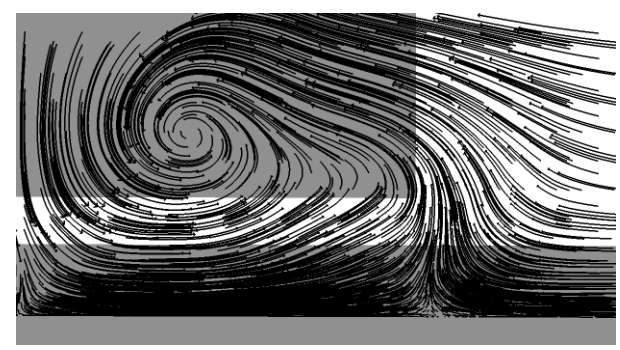

(c) $\mathrm{x} / \mathrm{H}=3$
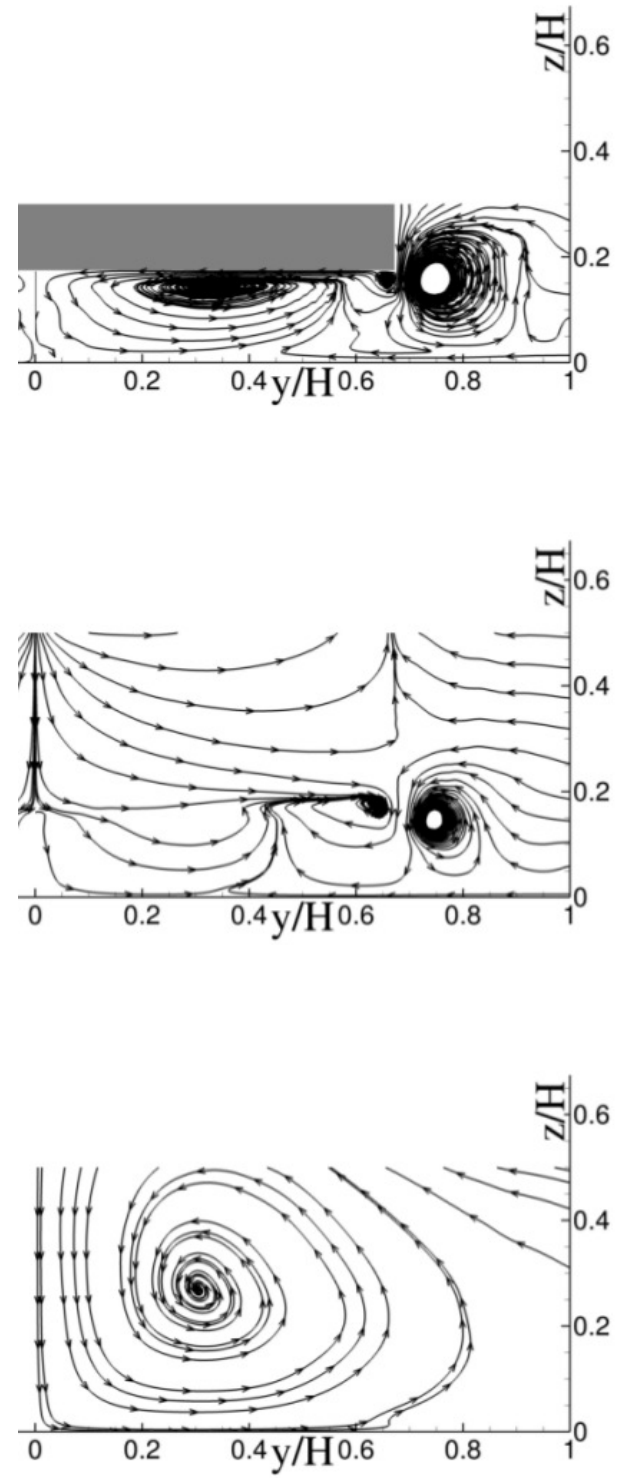

Figure 4.11: Surface projected streamlines from different $\mathrm{x} / \mathrm{H}$ locations, left from LES simulation at $\mathrm{Re}=0.5 \mathrm{E} 6$, right from $\mathrm{LES}$ at $\mathrm{Re}=0.5 \mathrm{E} 6[21]$

the power spectrum of these points is shown in Figure 4.13 versus the Strouhal number, which is defined in Section 1.1.1. Both data sets have distinct peaks around a Strouhal number of $S t=0.127$. This low frequency shedding of the wake ring vortices 
has also been found via Eichinger and Duell, at Strouhal numbers of $S t=0.128$ and $S t=0.11$ respectively $[21,36]$.

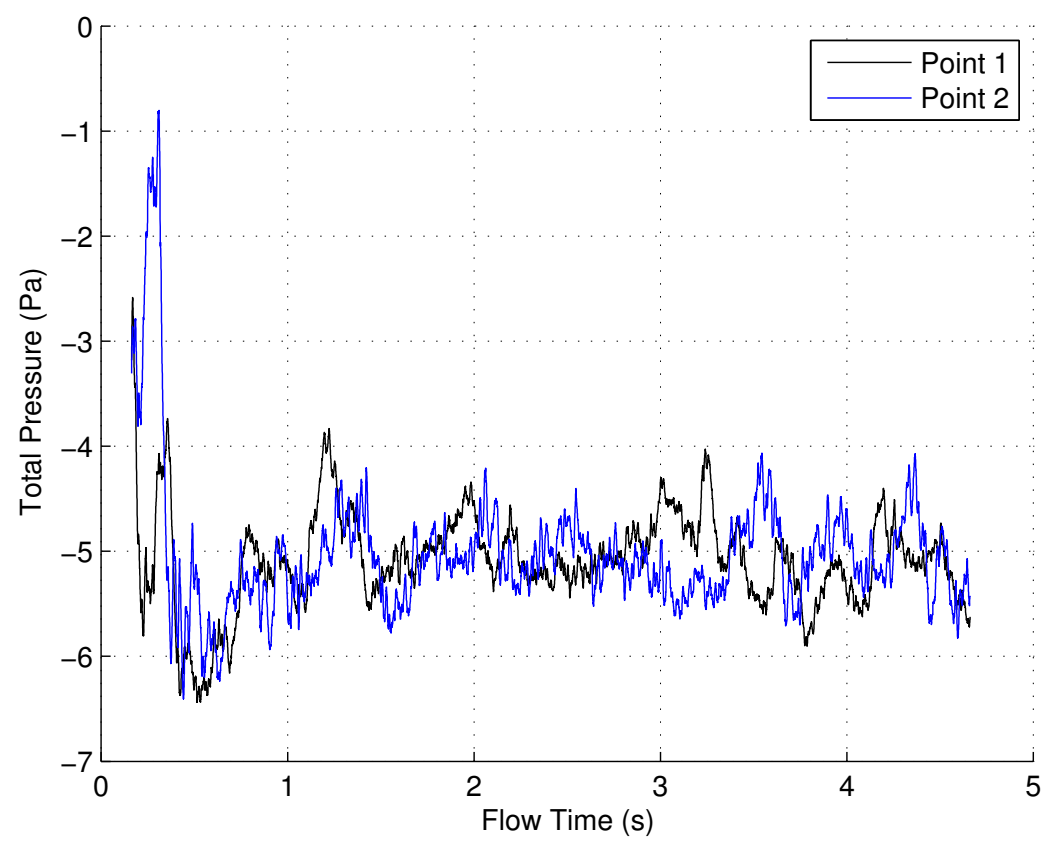

Figure 4.12: Unsteady total pressure signal from monitor points 1 and 2

It can be seen from Figure 4.9 that a relatively long sampling time is required of the averaged field to see the expected lateral symmetry of the flow. This is related to the lateral movement of the wake, as it can be seen from the instantaneous recirculation areas shown in Figure 4.14 at $t=2.22$ and $t=3.74$ respectively.

A visualization of the vortical structures is given in Figure 4.15 with the Qcriterion. One can see that the generation of the vortices starts immediately downstream of the rear edges, and that these vortices are parallel to the edges. The tubular shape in the lower corner shows similar vortex formation to that mentioned in Figure 4.11. Near the model rear the vortices are small and velocity is low, however as they 


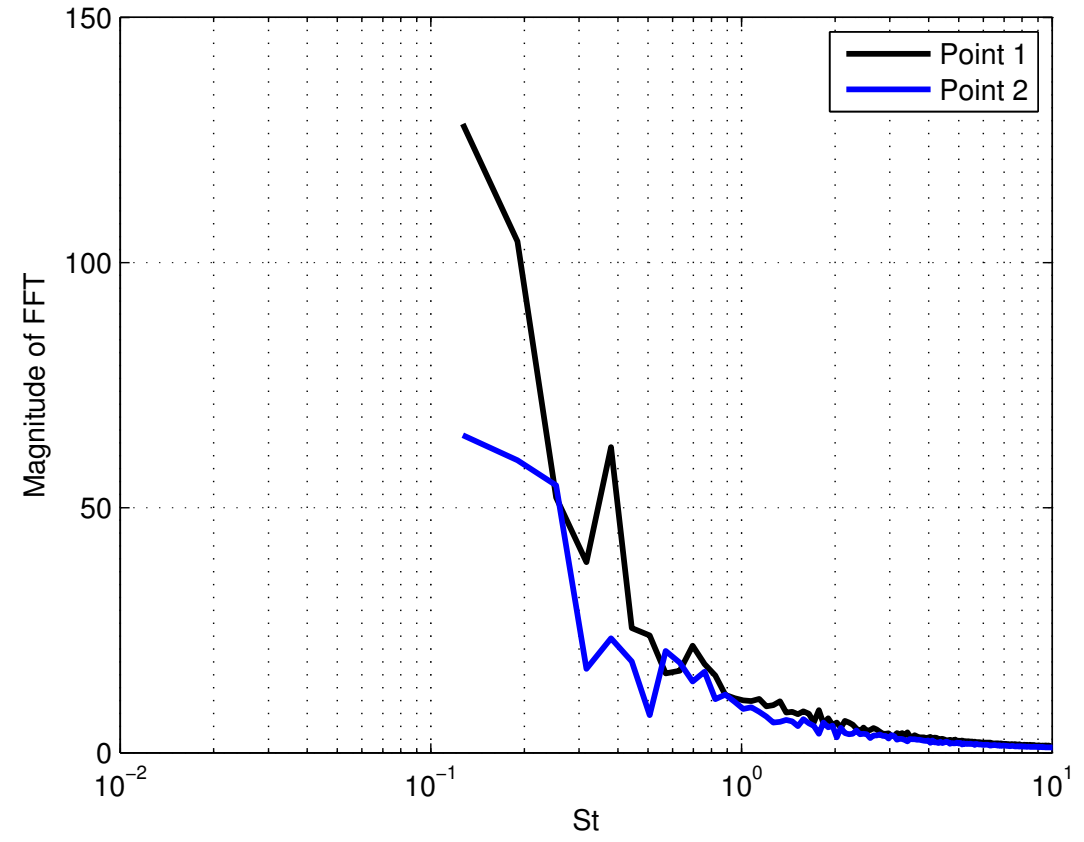

Figure 4.13: Fast Fourier Transform of monitor points 1 and 2

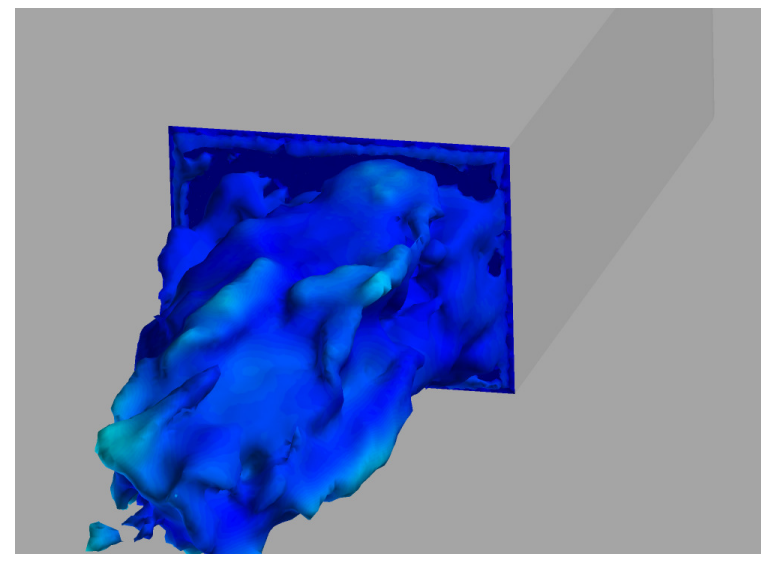

(a) $\mathrm{t}=2.22$

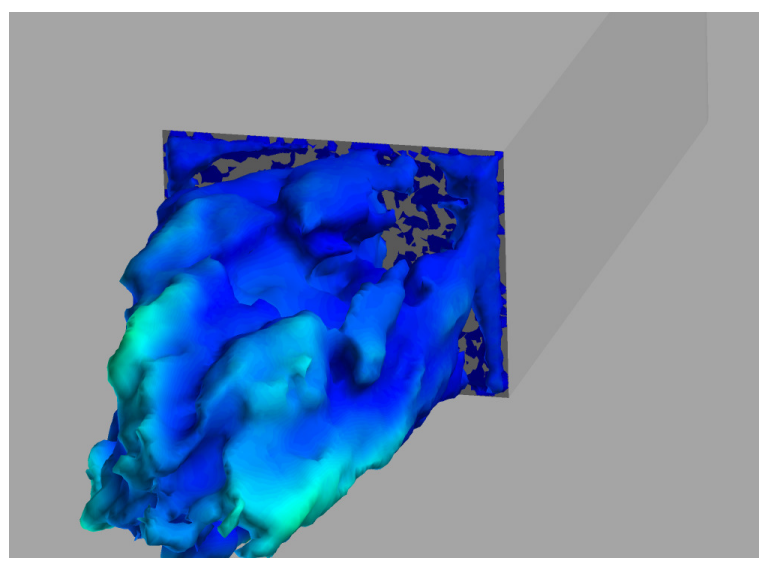

(b) $\mathrm{t}=3.74$

Figure 4.14: Instantaneous reverse flow plot isosurfaces in the vehicle wake colored by pressure (airflow inside shaded region has negative $\mathrm{x}$-velocity) 
convect downstream the velocity increases, increasing both the magnitude and size of the vortices correspondingly.

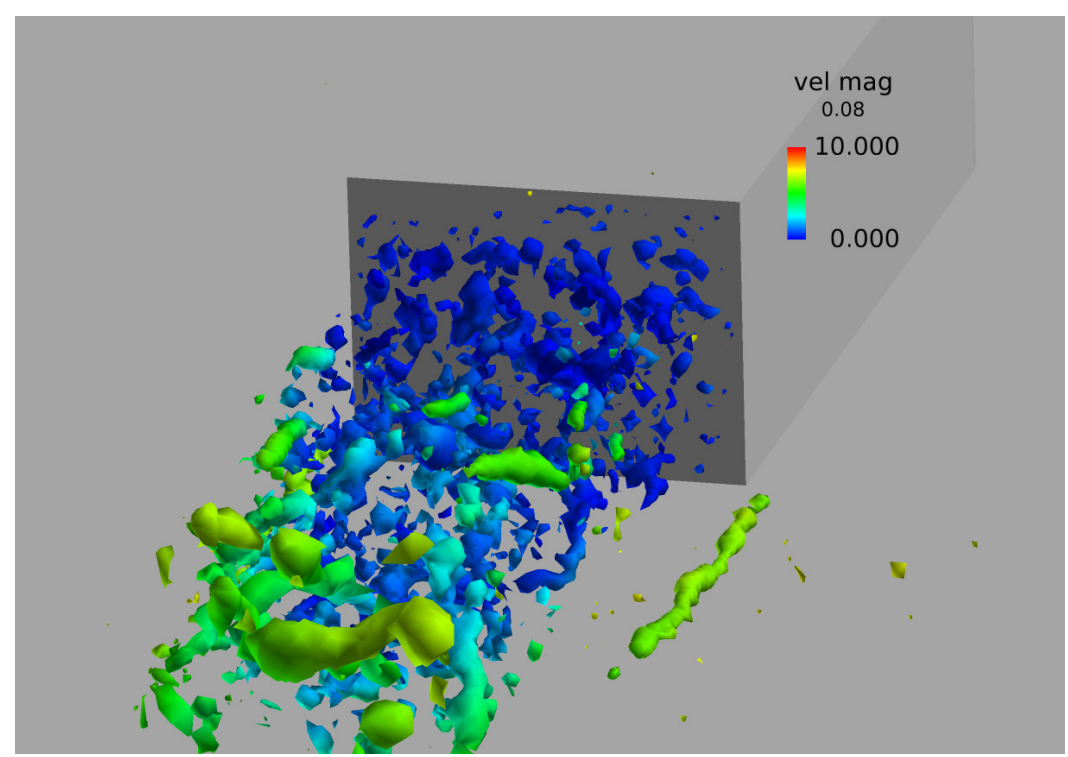

Figure 4.15: Q-Criterion iso-surfaces in the wake of the vehicle model, colored by local velocity magnitude

\subsection{Suction Control Case}

For this control scheme a set of suction jets are utilized. The controls are distributed along each of the four sides of the body with normals facing the outer boundaries, as shown in Figure 4.16. The suction slits are $0.017 \mathrm{H}$ wide and the same distance from all edges. A constant suction jet of $1.0 U_{\infty}$ is applied uniformly across the entire slit. Besides a slight local variation in the mesh around the slits, everything about this controlled case is identical to the previous base case including time step, numerical schemes, and initial conditions. 


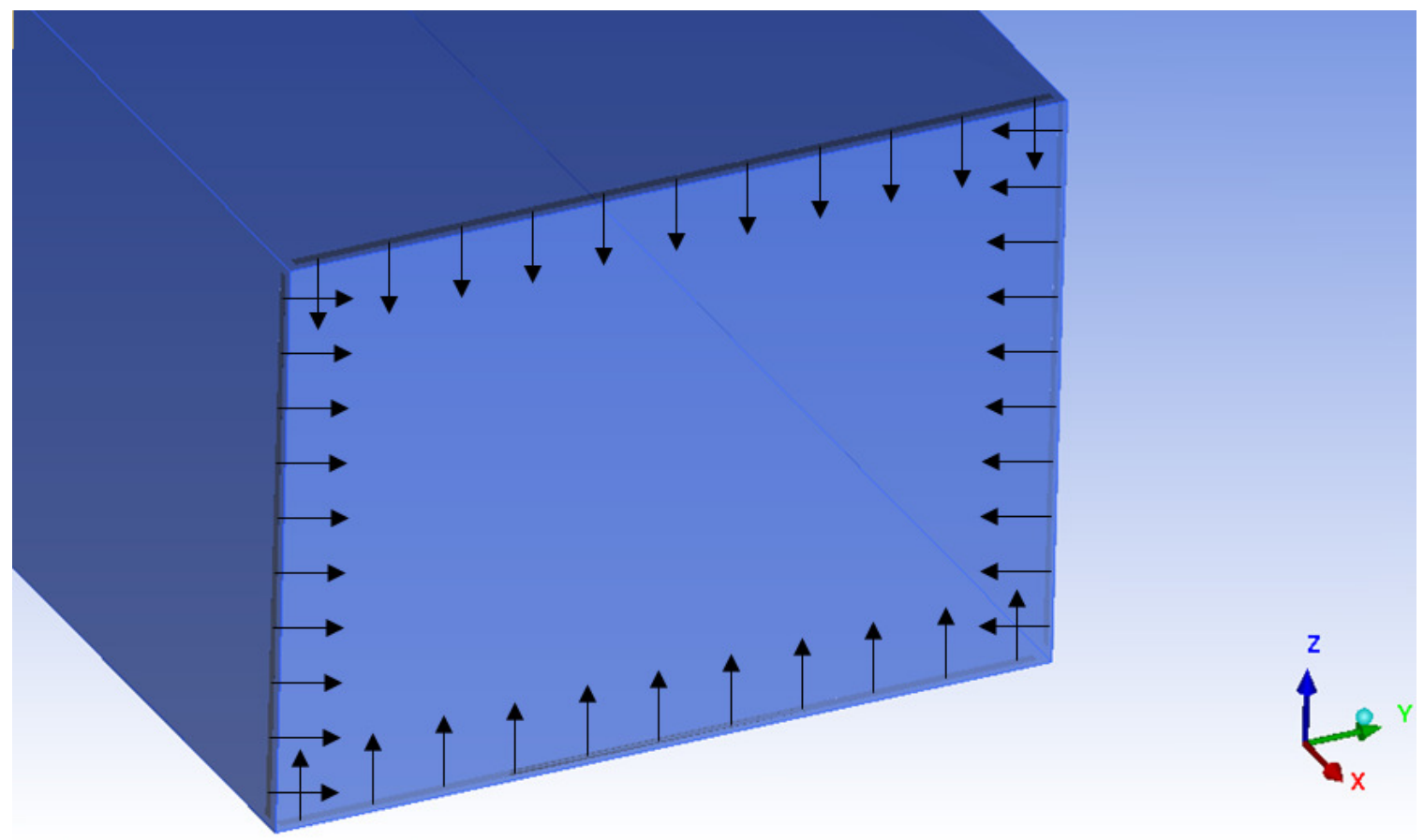

Figure 4.16: Suction Control Scheme Implementation

Figure 4.17 shows plane-projected time-averaged streamlines of this suction case compared with the previous case without control. It is critical to see that the dominant ring-shaped vortex structure still remains, however the overall wake shedding size has been reduced. Since Figure 4.17 shows both sets of streamlines at equal resolutions and scales, it is clear to see that the general wake area is reduced by approximately $10 \%$.

This conclusion is also supported by Figure 4.18, which represents an instantaneous screen shot of the general wake area by showing isosurfaces of reverse flow colored by velocity magnitude. Equivalent scales are used here to make it easy to notice a similar reduction in general wake area as is postulated from Figure 4.17.

In Figure 4.19 the surface-projected streamlines based on average values are shown on the rear surface, in addition to the average pressure distribution (warmer colors 

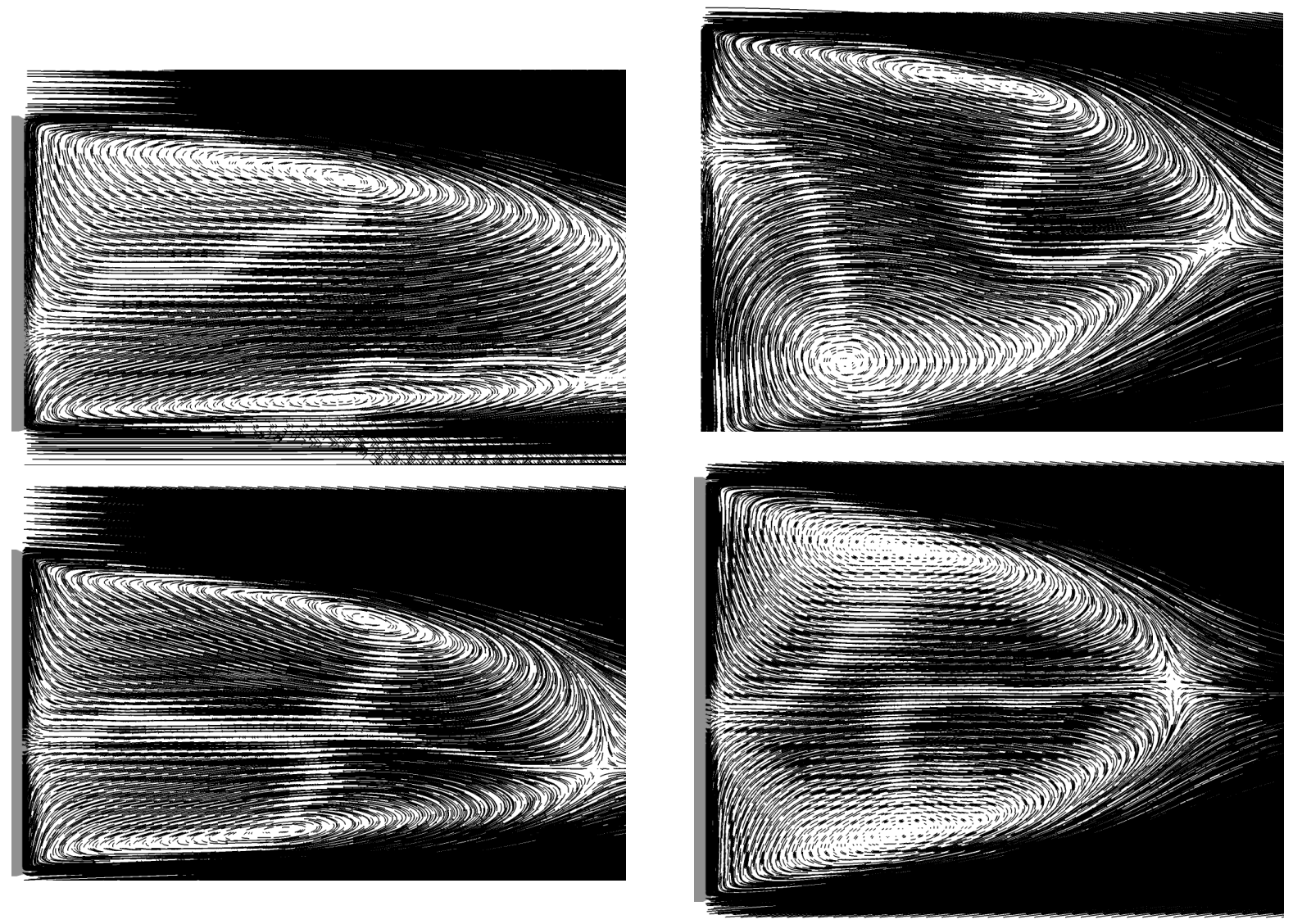

Figure 4.17: LES, Time-averaged, plane-projected, streamlines: (Top) Re $=4$ E6 (no control), (Bottom) Re $=4 \mathrm{E} 6$ (suction)

indicate the areas of higher pressure). Both sets of streamlines emanate from the stagnation point indicated by the streamline plot of Figure 4.17, although the slight variation in absolute location can again be attributed to averaging instabilities. The main takeaway from this analysis is to note the increase in back-pressure from the controlled case.

Approximate wake area and back-pressure are directly related to Chapter 1 and the discussions on how to reduce the aerodynamic drag of a bluff body in motion. The suction control has accomplished two of the three goals mentioned, reducing wake flow 


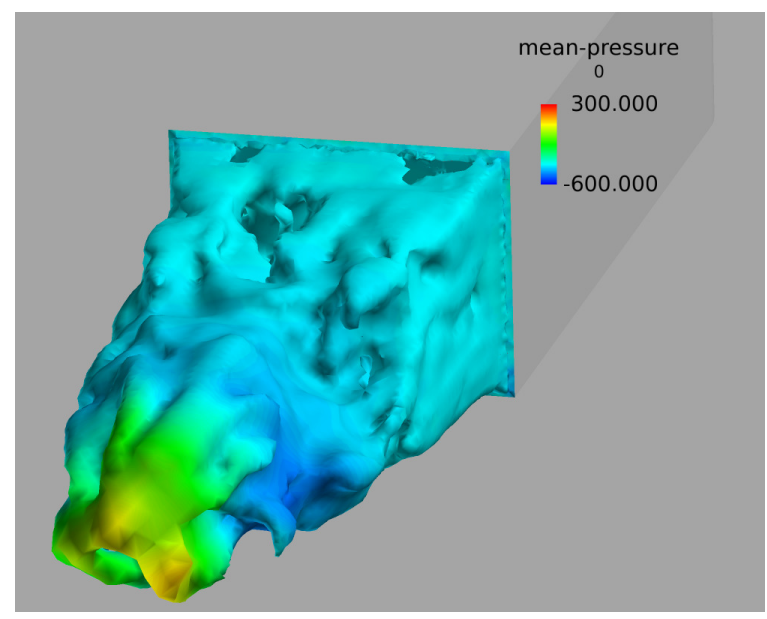

(a) $\operatorname{Re}=4 \mathrm{E} 6$ (no control)

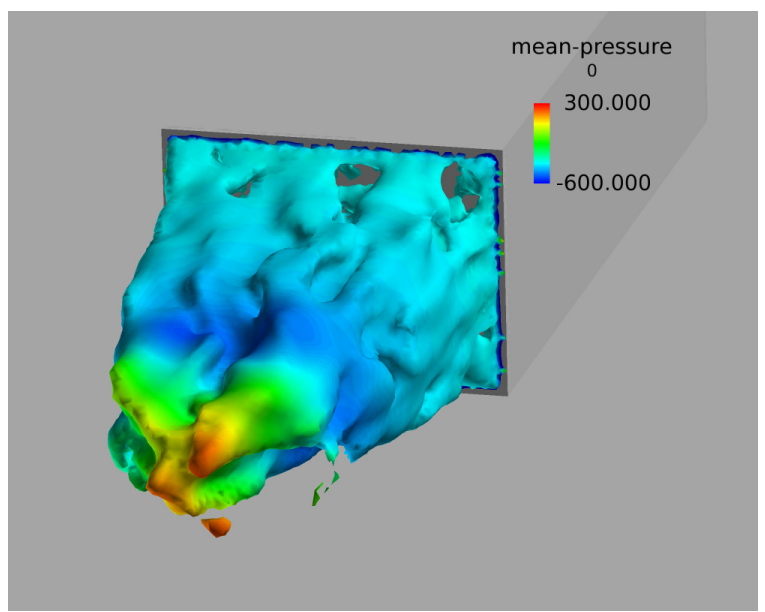

(b) $\operatorname{Re}=4 \mathrm{E} 6$ (suction)

Figure 4.18: Instantaneous reverse flow plot isosurfaces in the vehicle wake colored by pressure (airflow inside shaded region has negative $\mathrm{x}$-velocity)

size and increasing back-pressure. Coupled together, these effects are strong enough to reduce the aerodynamic drag of the bluff body model as is shown in Figure 4.20, by an average of $10 \%$. 


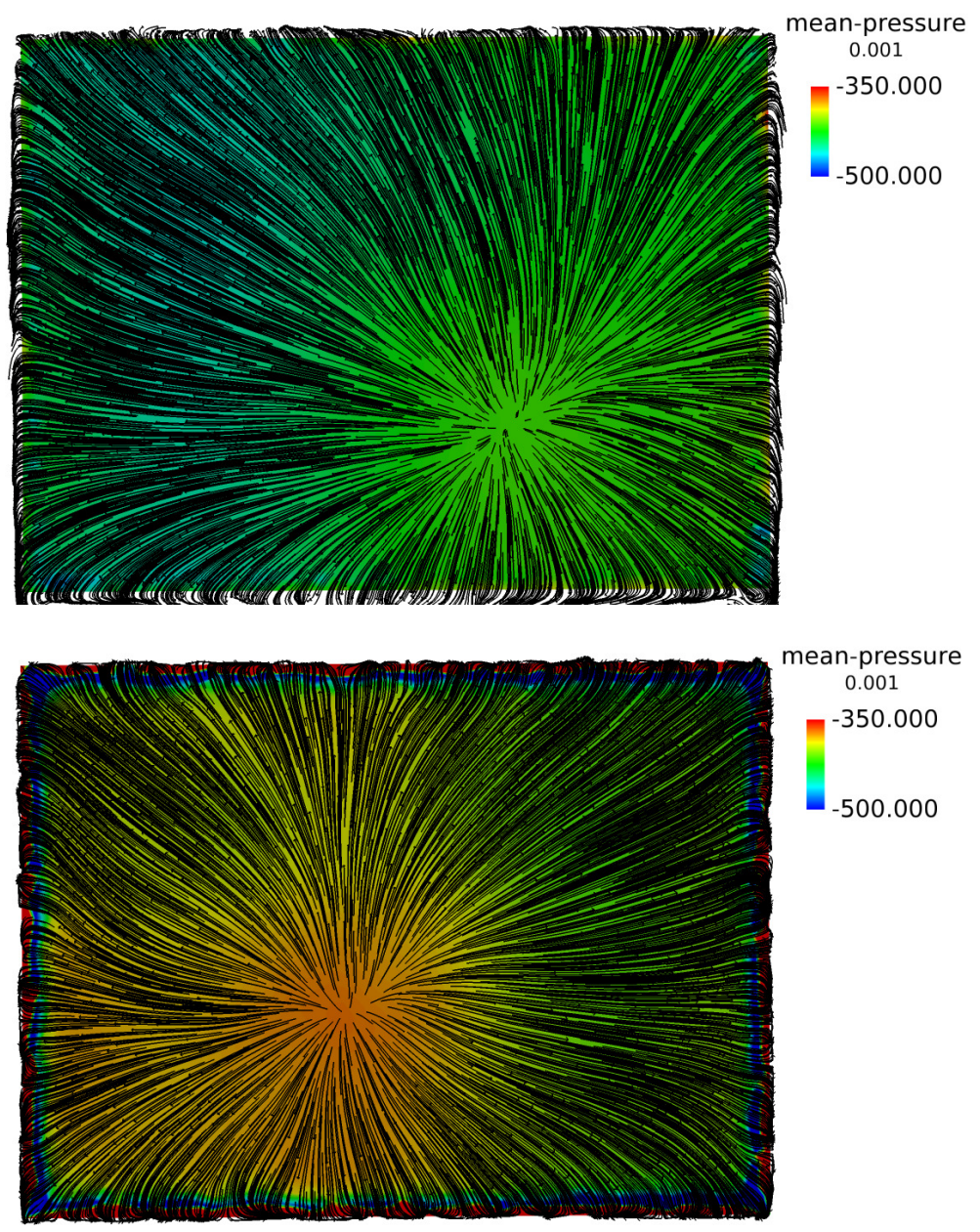

Figure 4.19: Pressure distribution and streamlines projected to the vehicle rear-end surface: $(\mathrm{Top}) \mathrm{Re}=4 \mathrm{E} 6$ (no control), (Bottom) Re $=4 \mathrm{E} 6$ (suction control) 


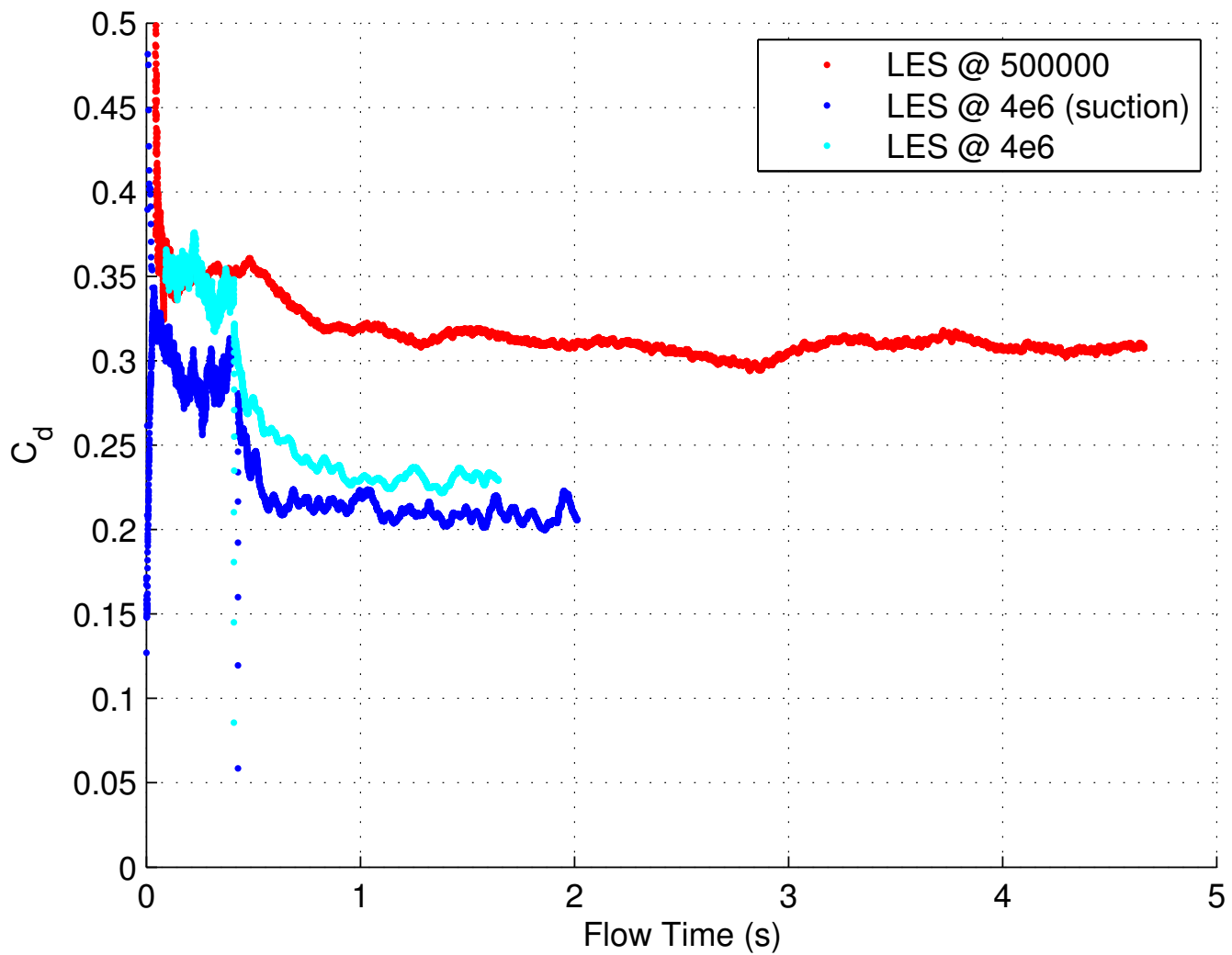

Figure 4.20: Transient drag coefficient for LES simulations 


\section{Chapter 5: Conclusions and Outlook}

\subsection{Concluding Remarks}

Active drag reduction on the zero-slant angle Ahmed body has been investigated on both the two-dimensional and three-dimensional scale. In the case of $2 \mathrm{D}$ the turbulence model $k-\epsilon \mathrm{RNG}$ was used to solve for the flow field and both suction and blowing control schemes were utilized. In regards to 3D, both a RANS model $(k-\omega$ SST) and the LES model were employed and only the suction control was tested.

Conclusions coming from the 2D simulations indicate that given a typical Reynolds number of one to four million, the suction and blowing jets will be effective at reducing the drag coefficient by increasing the Strouhal number until the point at which shedding completely stops. Though the methods by which each actuator achieves this shedding stoppage have different effects on the flow field, once this phenomenon occurs the drag coefficient sees a sharp decrease. The blowing jet creates a strong local increase in back-pressure that is able to more strongly affect the shedding and lower the drag at lower Reynolds numbers, however the suction control provides a more uniform increase of back-pressure and creates a higher magnitude drag decrease, albeit at a higher Reynolds number. In an attempt to couple these controls and gain 
the benefits of both at once, a secondary set of simulations was run in which both controls were active simultaneously. After comparison to the original single-use controls (at equivalent power) it was concluded that the coupling of both actuators actually produced a magnified drag reduction effect by employing the methods of both controls at once. A drag reduction of up to $75 \%$ from the base case has been recorded.

Simulations in regards to the $3 \mathrm{D}$ cases are not as extensive as $2 \mathrm{D}$ due to computational constraints, however important insights were still noted. By utilizing an extension of the suction jets into a slit along each side wall of the rear, the drag coefficient of the base case can be reduced. The drag coefficient reduction in the 3D case is caused by an altered flow field from the suction control. However, the added dimension causes additional 3D effects that limit the effectiveness of this suction control. Effectively, the controls reduce the size and magnitude of the near-body vortices and create a more streamlined flow field in the wake of the Ahmed model. This new altered flow field gives rise to an average drag reduction of $10 \%$.

\subsection{Outlook}

The research presented here is by no means a completely exhaustive endeavor into active controlled drag reduction of bluff bodies. Additional studies into utilizing this type of suction control at different incoming velocity fractions and Reynolds numbers will need to be performed, similar to that of the $2 \mathrm{D}$ simulations, in order to discover

how the actuator will react to these kinds of changes and the resulting flow field with the added dimension. Similar to the 2D discussion on multiple simultaneous actuators presented here, a study employing both suction and blowing jets, or even passive control devices such as boat tails or porous mediums, would yield valuable 
information as to how these kinds of actuators would interact with each other. In addition, physical implementation of such controls could lead to improved efficiency due to innate improvements in the actuator construction or redirection of suction flow for blowing use to couple the systems. A power efficiency study would produce valuable insights into at exactly what point the addition of more powerful controls is no longer beneficial to the overall power saving features of the control scheme itself. Specifically, usage of the open source Greenhouse Gas Emissions Model (GEM) from the EPA would allow for extensive research into fuel savings for an array of altered drag coefficients. 
Appendix A: Additional Data from 2-D Cases 


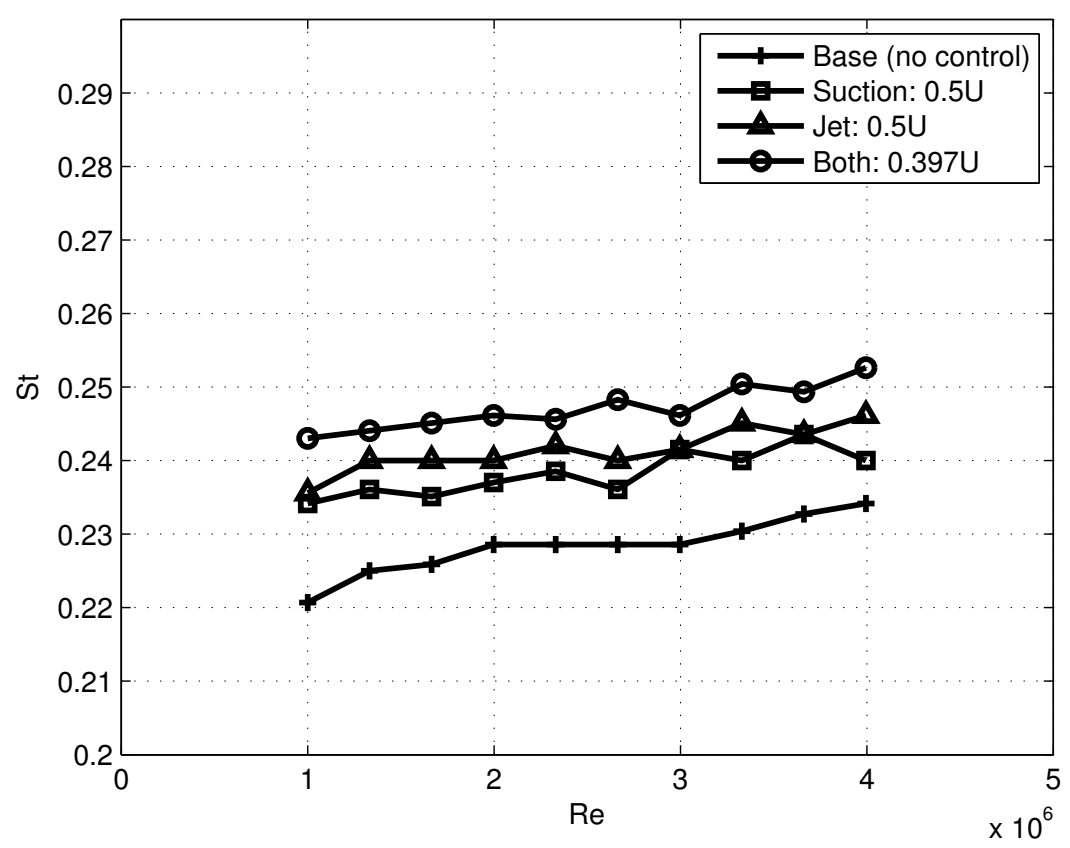

Figure A.1: Strouhal number data for equivalent power of $0.5 \cdot U_{\infty}$

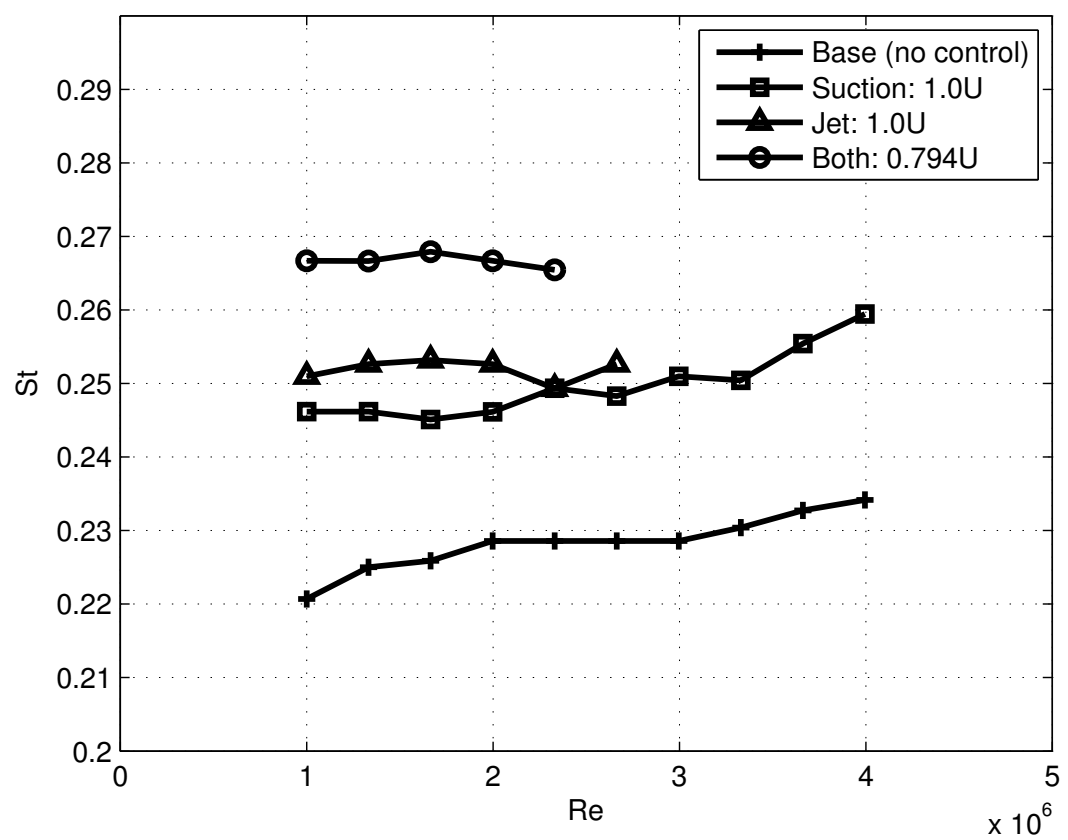

Figure A.2: Strouhal number data for equivalent power of $1.0 \cdot U_{\infty}$ 


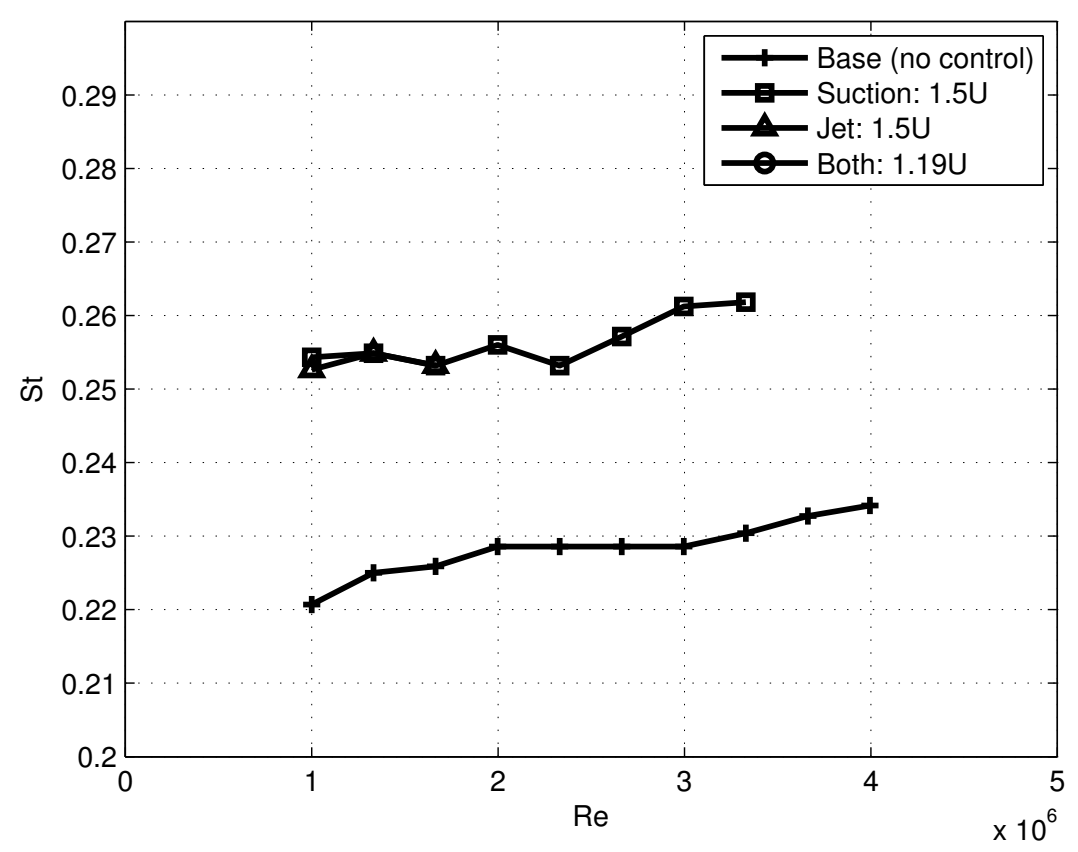

Figure A.3: Strouhal number data for equivalent power of $1.5 \cdot U_{\infty}$

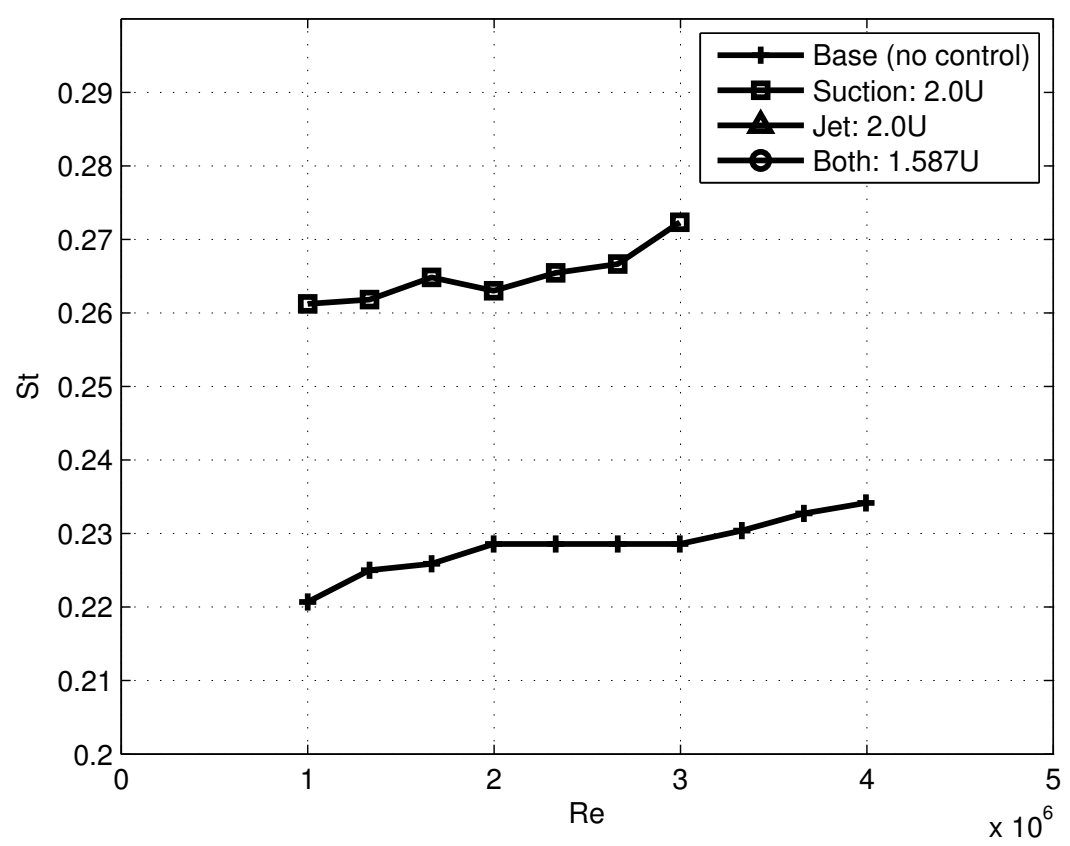

Figure A.4: Strouhal number data for equivalent power of $2.0 \cdot U_{\infty}$ 


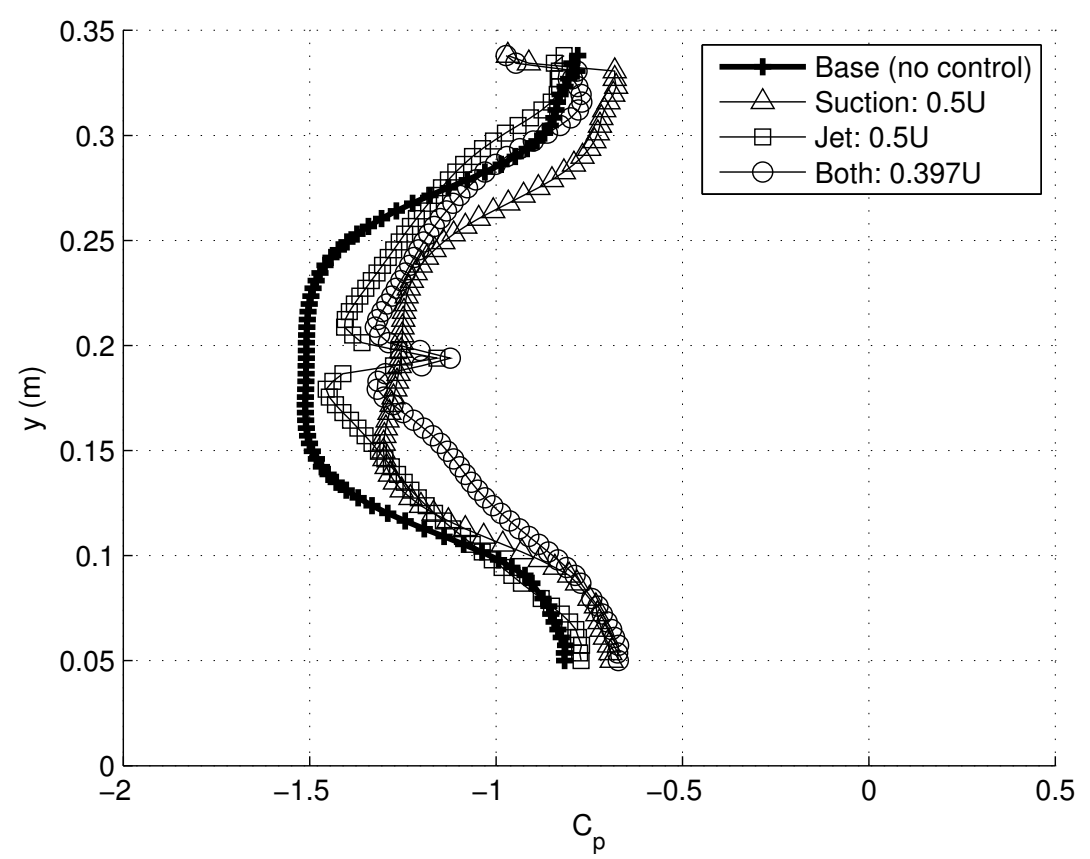

Figure A.5: Strouhal number data for equivalent power of $0.5 \cdot U_{\infty}$ at $\operatorname{Re}=2.33 \mathrm{E} 06$

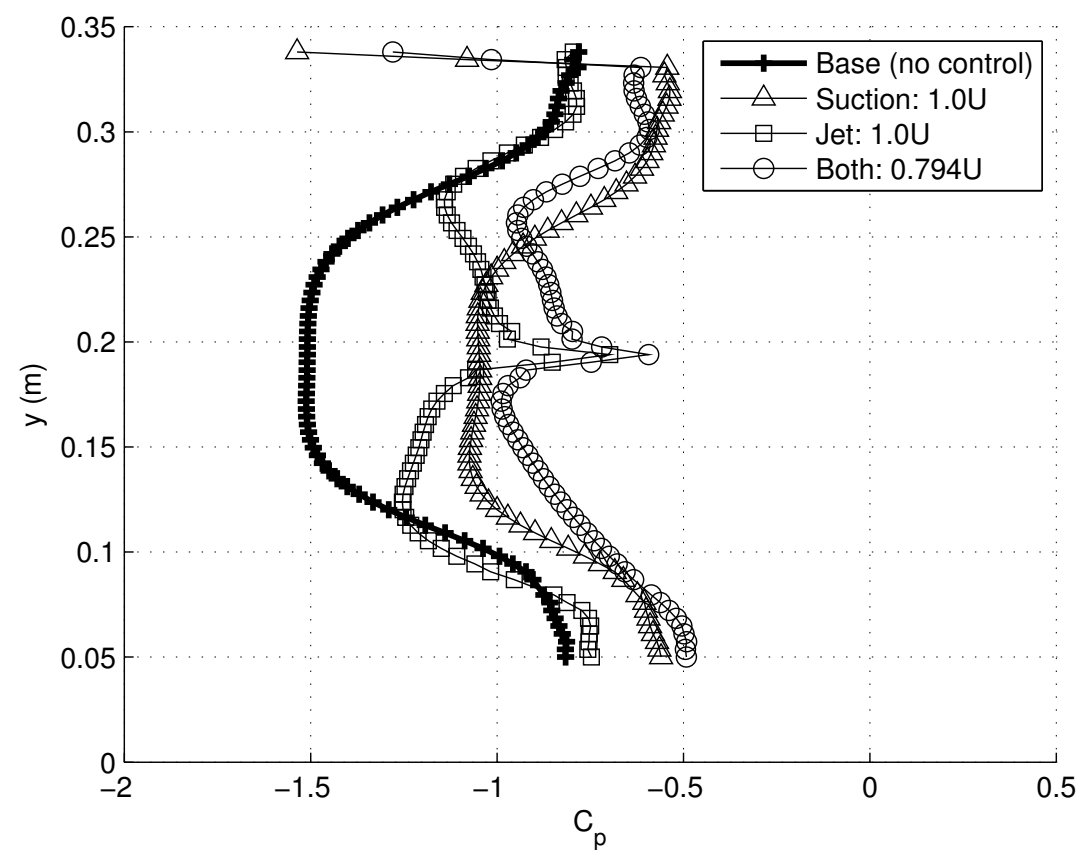

Figure A.6: Strouhal number data for equivalent power of $1.0 \cdot U_{\infty}$ at $\operatorname{Re}=2.33 \mathrm{E} 06$ 


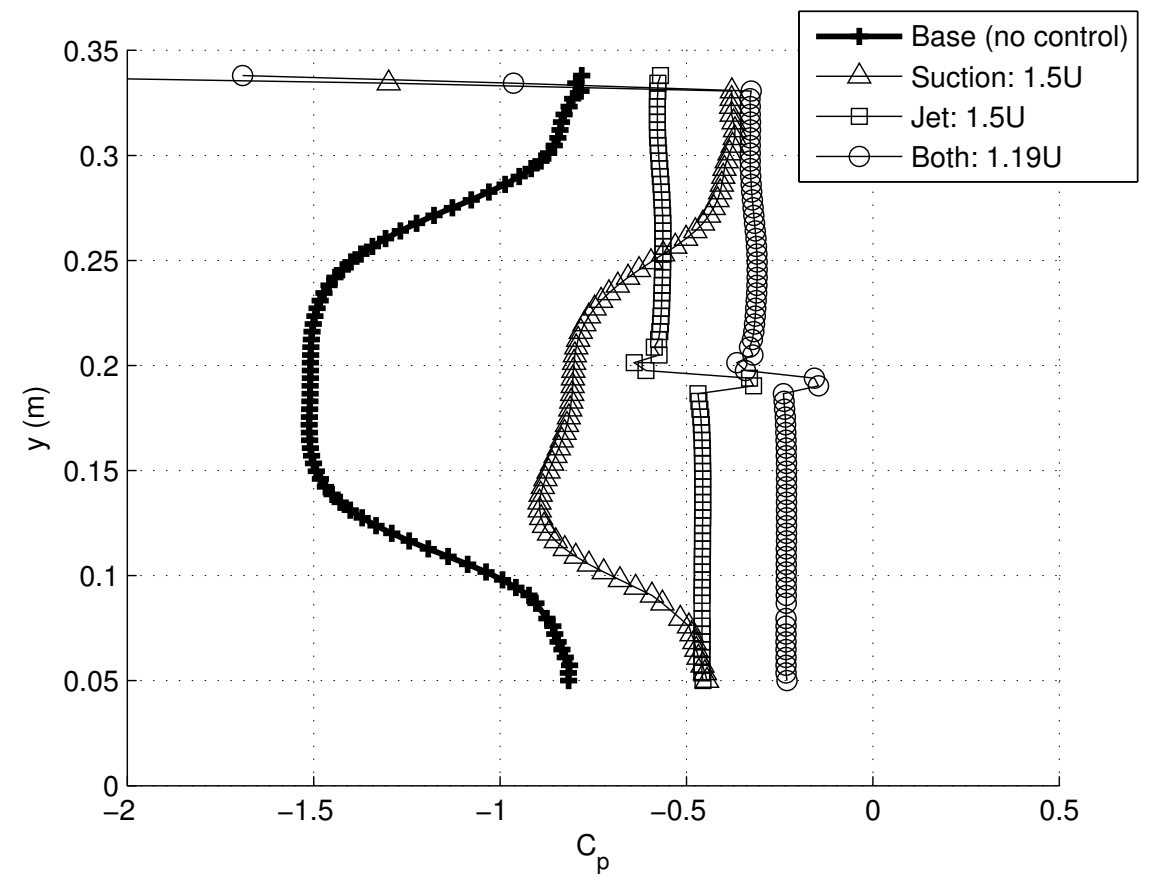

Figure A.7: Strouhal number data for equivalent power of $1.5 \cdot U_{\infty}$ at $\operatorname{Re}=2.33 \mathrm{E} 06$

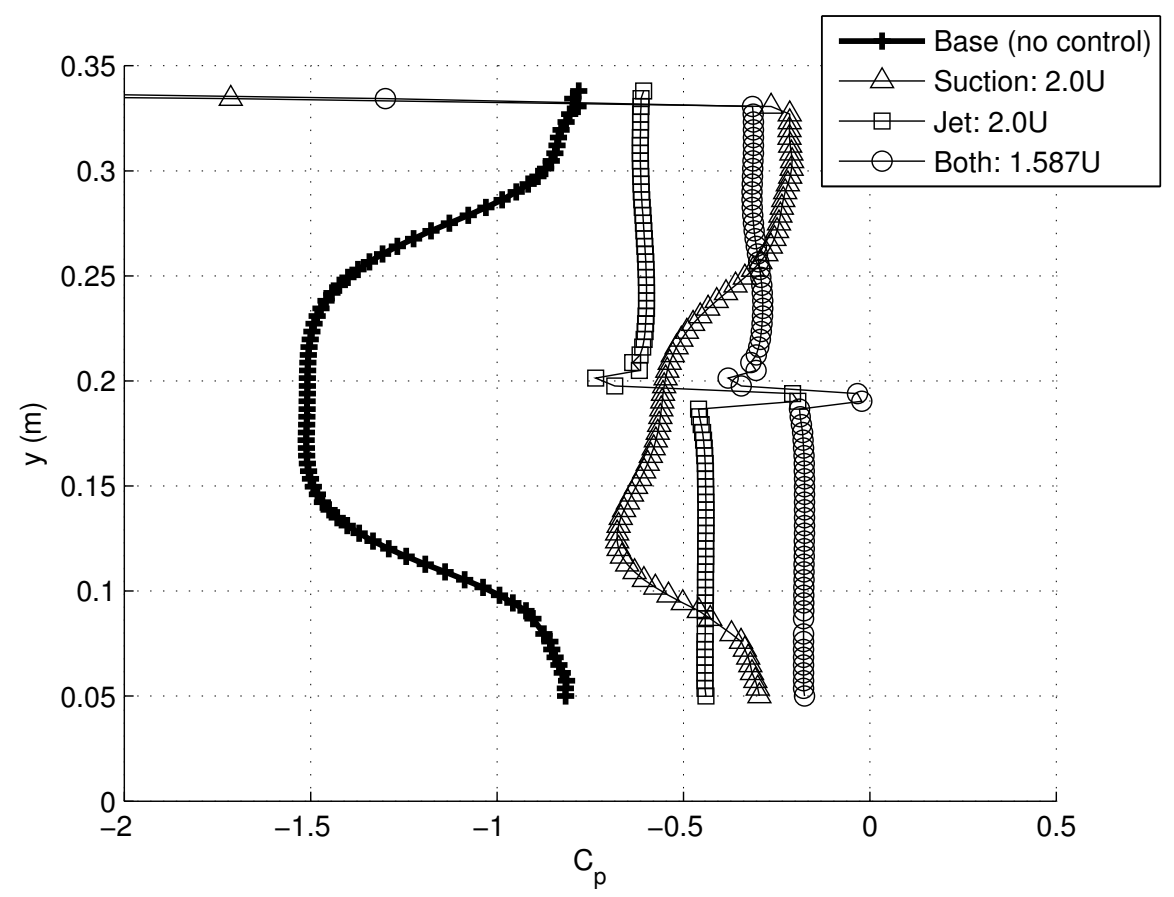

Figure A.8: Strouhal number data for equivalent power of $2.0 \cdot U_{\infty}$ at $\operatorname{Re}=2.33 \mathrm{E} 06$ 


\section{Bibliography}

[1] Ian J Sobey. Oscillatory flows at intermediate strouhal number in asymmetric channels. Journal of Fluid Mechanics, 125:359-373, 1982.

[2] Anatol Roshko. Experiments on the flow past a circular cylinder at very high reynolds number. Journal of Fluid Mechanics, 10(03):345-356, 1961.

[3] HH Bruun and POAL Davies. An experimental investigation of the unsteady pressure forces on a circular cylinder in a turbulent cross flow. Journal of Sound and Vibration, 40(4):535-559, 1975.

[4] CHK Williamson. Oblique and parallel modes of vortex shedding in the wake of a circular cylinder at low reynolds numbers. Journal of Fluid Mechanics, 206:579-627, 1989.

[5] Turgut Sarpkaya et al. In-line and transverse forces, on cylinders in oscillatory flow at high reynolds numbers. In Offshore Technology Conference. Offshore Technology Conference, 1976.

[6] O Almeida, S Mansur, and A Silveira-Neto. On the flow past rectangular cylinders: physical aspects and numerical simulation. Therm. Eng, 7(1):55-64, 2008.

[7] Alexey V Kozlov. Plasma actuators for bluff body flow control. PhD thesis, University of Notre Dame, 2010. 
[8] Hyungrok Do, Wookyung Kim, MG Mungal, and MA Cappelli. Bluff body flow separation control using surface dielectric barrier discharges. AIAA paper, 939:2007, 2007.

[9] Sarah Jo Haack. Flow control using plasma and synthetic jet actuators on bluff bodies. ProQuest, 2007.

[10] Adrien Thacker, S Aubrun, A Leroy, and Philippe Devinant. Effects of suppressing the $3 \mathrm{~d}$ separation on the rear slant on the flow structures around an ahmed body. Journal of Wind Engineering and Industrial Aerodynamics, 107:237-243, 2012.

[11] SR Ahmed, G Ramm, and G Faltin. Some salient features of the time-averaged ground vehicle wake. Technical report, SAE Technical Paper, 1984.

[12] SR Ahmed. Wake structure of typical automobile shapes. Journal of Fluids Engineering, 103(1):162-169, 1981.

[13] SR Ahmed. Influence of base slant on the wake structure and drag of road vehicles. Journal of fluids engineering, 105(4):429-434, 1983.

[14] T Morel. The effect of base slant on the flow pattern and drag of threedimensional bodies with blunt ends. In Aerodynamic Drag Mechanisms of Bluff Bodies and Road Vehicles, pages 191-226. Springer, 1978.

[15] Hermann Lienhart and Stefan Becker. Flow and turbulence structure in the wake of a simplified car model. Technical report, SAE Technical Paper, 2003. 
[16] RK Strachan, K Knowles, and NJ Lawson. The vortex structure behind an ahmed reference model in the presence of a moving ground plane. Experiments in fluids, 42(5):659-669, 2007.

[17] Patrick Gilliéron and Francis Chometon. Modelling of stationary threedimensional separated air flows around an ahmed reference model. In ESAIM: Proceedings, volume 7, pages 173-182. EDP Sciences, 1999.

[18] Sajjad Beigmoradi, Hassan Hajabdollahi, and Asghar Ramezani. Multi-objective aero acoustic optimization of rear end in a simplified car model by using hybrid robust parameter design, artificial neural networks and genetic algorithm methods. Computers \&f Fluids, 90:123-132, 2014.

[19] A Brunn, E Wassen, D Sperber, W Nitsche, and F Thiele. Active drag control for a generic car model. In Active Flow Control, pages 247-259. Springer, 2007.

[20] Charles-Henri Bruneau, Emmanuel Creusé, Delphine Depeyras, Patrick Gilliéron, and Iraj Mortazavi. Coupling active and passive techniques to control the flow past the square back ahmed body. Computers $\&$ Fluids, 39(10):1875$1892,2010$.

[21] Sandor Eichinger. Active flow separation control of ground transportation vehicle configurations. 2012.

[22] Mark Pastoor, Lars Henning, Bernd R Noack, Rudibert King, and Gilead Tadmor. Feedback shear layer control for bluff body drag reduction. Journal of fluid mechanics, 608:161-196, 2008. 
[23] Charles-Henri Bruneau, Iraj Mortazavi, and Patrick Gilliéron. Passive control around the two-dimensional square back ahmed body using porous devices. Journal of Fluids Engineering, 130(6):061101, 2008.

[24] Bahram Khalighi, S Zhang, C Koromilas, SR Balkanyi, Luis P Bernal, G Iaccarino, and P Moin. Experimental and computational study of unsteady wake flow behind a bluff body with a drag reduction device. Technical report, SAE Technical Paper, 2001.

[25] Fu-Hung Hsu and Roger L Davis. Design optimization of tractor-trailer drag reduction. In Collection of Technical Papers-AIAA Applied Aerodynamics Conference, San Antonio TX, 2009.

[26] Mathieu Rouméas, Patrick Gilliéron, and Azeddine Kourta. Drag reduction by flow separation control on a car after body. International journal for numerical methods in fluids, 60(11):1222-1240, 2009.

[27] Noele Peres and Richard Pasquetti. Numerical study of drag reduction of a car model by active control using mico-jets. In CFM 2013, 2013.

[28] Mathieu Roumeas, Patrick Gilliéron, and Azeddine Kourta. Analysis and control of the near-wake flow over a square-back geometry. Computers $\&$ Fluids, 38(1):60-70, 2009.

[29] Andrey Nikolaevich Kolmogorov. A refinement of previous hypotheses concerning the local structure of turbulence in a viscous incompressible fluid at high reynolds number. Journal of Fluid Mechanics, 13(01):82-85, 1962. 
[30] Chin-Hoh Moeng and PP Sullivan. Large eddy simulation. Encyclopedia of Atmospheric Sciences, 1140:1150, 2002.

[31] VSASTBCG Yakhot, SA Orszag, S Thangam, TB Gatski, and CG Speziale. Development of turbulence models for shear flows by a double expansion technique. Physics of Fluids A: Fluid Dynamics (1989-1993), 4(7):1510-1520, 1992.

[32] Florian R Menter. Zonal two equation k-turbulence models for aerodynamic flows. AIAA paper, 2906:1993, 1993.

[33] Luca Bruno and S Khris. The validity of $2 \mathrm{~d}$ numerical simulations of vortical structures around a bridge deck. Mathematical and computer modelling, $37(7): 795-828,2003$.

[34] Charles-Henri Bruneau, Emmanuel Creusé, Patrick Gilliéron, and Iraj Mortazavi. Effect of the vortex dynamics on the drag coefficient of a square back ahmed body: Application to the flow control. European Journal of MechanicsB/Fluids, 45:1-11, 2014.

[35] JL Graysmith, AJ Baxendale, JP Howell, and T Haynes. Comparisons between cfd and experimental results for the ahmed reference model. In $R A e S$ conference on vehicle aerodynamics, 1994.

[36] Edward G Duell and AR George. Experimental study of a ground vehicle body unsteady near wake. Technical report, SAE Technical Paper, 1999. 